\title{
Energy Density Assessment of Organic
}

\section{Batteries}

Xabier Judez, Lixin Qiao, Michel Armand, and Heng Zhang*

Electrical Energy Storage Department, CIC Energigune, Parque Tecnológico de Álava, Albert Einstein 48, 01510 Miñano, Álava, Spain

\section{AUTHOR INFORMATION}

\section{Corresponding Author}

*E-mail: hzhang@cicenergigune.com (H.Z.)

\section{Table of content}

1. Brief introduction on the cell chemistry of organic batteries

Page S2

2. State-of-art on organic batteries

Page S3

3. Enlarged figures for the state-or-art on organic batteries....

Page S34

4. Energy density calculation details Page S36

5. Scilab codes for energy density calculation Page S40

6. References Page S69 


\section{Brief introduction on the cell chemistry of organic batteries}

Table S1 Redox mechanisms and properties of the different organic electrodes.

\begin{tabular}{|c|c|c|c|c|}
\hline Type & Redox mechanisms & Pros & Cons & Example \\
\hline $\begin{array}{l}\text { Conjugated } \\
\text { polymers }\end{array}$ & $\begin{array}{l}(\mathrm{R})^{\mathrm{x}+} \leftrightarrow(\mathrm{R}) \frac{)^{\mathrm{n}}}{\mathrm{n}} \\
(\mathrm{R}))_{\mathrm{n}} \leftrightarrow\left(\mathrm{R} \frac{)^{\mathrm{y}}}{\mathrm{n}}\right.\end{array}$ & $\begin{array}{l}\text { High } \\
\text { electronic } \\
\text { conductivity }\end{array}$ & Sloping voltage curves & \\
\hline $\begin{array}{l}\text { Carbonyl- } \\
\text { based polymer }\end{array}$ & 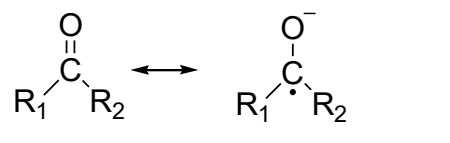 & $\begin{array}{l}\text { High capacity, } \\
\text { fast kinetics }\end{array}$ & $\begin{array}{l}\text { Highly soluble in organic } \\
\text { solvents, low electronic } \\
\text { conductivity }\end{array}$ & \\
\hline $\begin{array}{l}\text { Sulfur- } \\
\text { containing } \\
\text { compounds }\end{array}$ & $R_{1}-S-S-R_{2} \longleftrightarrow R_{1}-S^{-}+R_{2}-S$ & High capacity & $\begin{array}{l}\text { Highly soluble in organic } \\
\text { solvents, low kinetics and } \\
\text { electronic conductivity }\end{array}$ & \\
\hline $\begin{array}{l}\text { Radical } \\
\text { compounds }\end{array}$ & 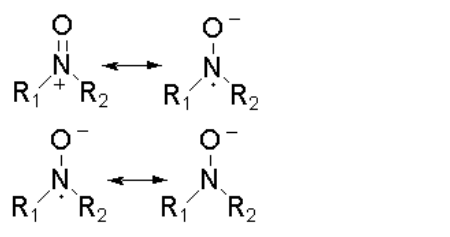 & $\begin{array}{l}\text { Fast kinetics, } \\
\text { flat plateau }\end{array}$ & $\begin{array}{l}\text { Low capacity, lowly } \\
\text { conductive, high self- } \\
\text { discharge behavior, highly } \\
\text { soluble in organic solvents }\end{array}$ & \\
\hline Others & $\mathrm{R}_{1}-\mathrm{N}=\mathrm{N}-\mathrm{R}_{2} \longleftrightarrow \mathrm{R}_{1}-\overline{\mathrm{N}}-\mathrm{N}-\mathrm{R}_{2}$ & $\begin{array}{l}\text { High capacity, } \\
\text { fast kinetics }\end{array}$ & $\begin{array}{l}\text { Highly soluble in organic } \\
\text { solvents, low electronic } \\
\text { conductivity }\end{array}$ & \\
\hline
\end{tabular}




\section{State-of-art on organic batteries}

Table S2. Properties of organic materials developed for Li-based batteries. ${ }^{\text {a) }}$

\begin{tabular}{|c|c|c|c|c|c|c|c|c|c|}
\hline Number & Structure & $\begin{array}{l}\text { Specific capacity } \\
\text { b) } / \mathrm{mAh} \mathrm{g}^{-1}\end{array}$ & Cathode composition $^{\mathrm{c})}$ & $\begin{array}{l}\text { AM loading d) / } \\
\mathrm{mg} \mathrm{cm}^{-2}\end{array}$ & Anode & Electrolyte & $\begin{array}{l}\text { Voltage }^{\text {e) }} / \mathrm{V} \\
\text { vs. } \mathrm{Li}^{-\mathrm{Li}^{+}}\end{array}$ & Cyclability / $\mathrm{mAh} \mathrm{g}^{-1}$ & Ref \\
\hline \multicolumn{10}{|c|}{ Conjugated polymers } \\
\hline 1 & & & $\begin{array}{l}1 \text { (30) / Graphene (60) / } \\
\text { PVDF (10) }\end{array}$ & & $\mathrm{Li}$ & $\begin{array}{l}1 \mathrm{M} \text { LiTFSI in } \\
\text { DOL/DME }\end{array}$ & 2.20 & $\begin{array}{l}395\left(1^{\text {st }} \text { cycle }\right) \text { vs. } 324 \\
\left(200^{\text {th }} \text { cycle }\right) \text { at } 1 \mathrm{C}\end{array}$ & 1 \\
\hline 2 & & & $\begin{array}{l}2(10) \text { / Graphite (70) / } \\
\text { PVDF (20) }\end{array}$ & 0.71 & $\mathrm{Li}$ & $\begin{array}{l}1 \mathrm{M} \mathrm{LiPF}_{6} \text { in } \\
\mathrm{EC} / \mathrm{DEC}\end{array}$ & 3.60 & $\begin{array}{l}103\left(1^{\text {st }} \text { cycle }\right) \text { vs. } 68\left(40^{\text {th }}\right. \\
\text { cycle }) \text { at } 0.3 \mathrm{C}\end{array}$ & 2 \\
\hline 3 & & & 3 & & $\mathrm{Li}$ & $\begin{array}{l}1 \mathrm{M} \mathrm{LiPF}_{6} \text { in } \\
\mathrm{EC} / \mathrm{DMC} / \mathrm{EMC}\end{array}$ & 3.80 & $\begin{array}{l}80\left(1^{\text {st }} \text { cycle }\right) \text { vs. } 70\left(100^{\text {th }}\right. \\
\text { cycle }) \text { at } 40 \mathrm{~mA} \mathrm{~g}^{-1}\end{array}$ & 3 \\
\hline 4 & & 84 & $\begin{array}{l}4(60) / \mathrm{CB}(30) / \mathrm{PTFE} \\
(10)\end{array}$ & & $\mathrm{Ag} / \mathrm{AgCl}$ & $\begin{array}{l}1 \mathrm{M} \mathrm{LiBF}_{4} \text { in } \\
\mathrm{EC} / \mathrm{DMC}\end{array}$ & 3.00 & $\begin{array}{l}80\left(1^{\text {st }} \text { cycle }\right) \text { vs. } 64 \\
\left(30000^{\text {th }} \text { cycle }\right) \text { at } 1 \mathrm{~mA} \\
\mathrm{~cm}^{-2}\end{array}$ & 4 \\
\hline 5 & & 443 & $\begin{array}{l}5(60) / \text { Carbon (30) / } \\
\text { PVDF (10) }\end{array}$ & $5-10$ & $\mathrm{Li}$ & $\begin{array}{l}1 \mathrm{M} \mathrm{LiPF}_{6} \text { in } \\
\mathrm{EC} / \mathrm{DEC} / \mathrm{DMC}\end{array}$ & 2.20 & $\begin{array}{l}80\left(1^{\text {st }} \text { cycle }\right) \text { vs. } \sim 110\left(50^{\text {th }}\right. \\
\text { cycle }) \text { at } 0.1 \mathrm{C}\end{array}$ & 5 \\
\hline \multirow{2}{*}{6} & & & $\begin{array}{l}6(40) / \mathrm{CB}(40) / \text { Teflon } \\
(10)\end{array}$ & & $\mathrm{Li}$ & $\begin{array}{l}1 \text { M LiTFSI in } \\
\text { DOL/DME }\end{array}$ & 2.20 & $\begin{array}{l}880\left(7^{\text {th }} \text { cycle }\right) \text { vs. } 458 \\
\left(32^{\text {th }} \text { cycle }\right) \text { at } 50 \mathrm{~mA} \mathrm{~g}^{-1}\end{array}$ & 6 \\
\hline & & & $\begin{array}{l}\text { 6(30) / CC (60) / PVDF } \\
(10)\end{array}$ & & $\mathrm{Li}$ & $\begin{array}{l}1 \mathrm{M} \mathrm{LiPF}_{6} \text { in } \\
\mathrm{EC} / \mathrm{DEC}\end{array}$ & 3.60 & $\begin{array}{l}79\left(1^{\text {st }} \text { cycle }\right) \text { vs. } 61\left(50^{\text {th }}\right. \\
\text { cycle) at } 0.05 \mathrm{~mA} \mathrm{~g}^{-1}\end{array}$ & 7 \\
\hline 7 & & 214 & $\begin{array}{l}7(60) / \mathrm{AB}(30) / \mathrm{PVDF} \\
(20)\end{array}$ & 1.24 & $\mathrm{Li}$ & $\begin{array}{l}1 \mathrm{M} \mathrm{LiPF}_{6} \text { in } \\
\mathrm{EC} / \mathrm{DMC}\end{array}$ & 2.40 & $\begin{array}{l}147\left(1^{\text {st }} \text { cycle }\right) \text { vs. } 91\left(50^{\text {th }}\right. \\
\text { cycle }) \text { at } 100 \mathrm{~mA} \mathrm{~g}^{-1}\end{array}$ & 8 \\
\hline 8 & & 130 & $\begin{array}{l}\mathbf{8}(50) / \mathrm{AB}(40) / \mathrm{PVDF} \\
(10)\end{array}$ & & $\mathrm{Li}$ & $\begin{array}{l}1 \mathrm{M} \mathrm{LiPF}_{6} \text { in } \\
\mathrm{EC} / \mathrm{DMC}\end{array}$ & 3.50 & $\begin{array}{l}129\left(1^{\text {st }} \text { cycle }\right) \text { vs. } 110\left(50^{\text {th }}\right. \\
\text { cycle }) \text { at } 20 \mathrm{~mA} \mathrm{~g}^{-1}\end{array}$ & 9 \\
\hline
\end{tabular}


Table S2 (continued)

\begin{tabular}{|c|c|c|c|c|c|c|c|c|c|}
\hline Number & Structure & $\begin{array}{l}\text { Specific capacity } \\
\text { b) } / \mathrm{mAh} \mathrm{g}^{-1}\end{array}$ & Cathode composition $^{\mathrm{c}}$ & $\begin{array}{l}\text { AM loading d) / } \\
\mathrm{mg} \mathrm{cm}^{-2}\end{array}$ & Anode & Electrolyte & $\begin{array}{l}\text { Voltage } e^{\text {e) }} / \mathrm{V} \\
\text { vs. Li/Li }{ }^{+}\end{array}$ & Cyclability / $\mathrm{mAh} \mathrm{g}^{-1}$ & Ref \\
\hline 9 & & & $\begin{array}{l}9(70) / \mathrm{AB}(20) / \mathrm{CMC} \\
(10)\end{array}$ & 1 & $\mathrm{Li}$ & $\begin{array}{l}1 \mathrm{M} \mathrm{LiPF}_{6} \text { in } \\
\mathrm{EC} / \mathrm{DMC}\end{array}$ & 0.90 & $\begin{array}{l}1818\left(1^{\text {st }} \text { cycle }\right) \text { vs. } 580 \\
\left(1414^{\text {th }} \text { cycle }\right) \text { at } 500 \mathrm{~mA} \\
\mathrm{~g}^{-1}\end{array}$ & 10 \\
\hline 10 & & 1926 & $\begin{array}{l}\mathbf{1 0}(70) / \text { CNT (20)/ } \\
\text { PVDF (10) }\end{array}$ & & $\mathrm{Li}$ & $\begin{array}{l}1 \mathrm{M} \mathrm{LiPF}_{6} \text { in } \\
\mathrm{EC} / \mathrm{DEC}\end{array}$ & 1.15 & $\begin{array}{l}1787\left({ }^{\text {st }} \text { cycle }\right) \text { vs. } 1181 \\
\left(50^{\text {th }} \text { cycle }\right) \text { at } 100 \mathrm{~mA} \mathrm{~g}^{-1}\end{array}$ & 11 \\
\hline 11 & & 1802 & $\begin{array}{l}11(70) / \text { CNT (20)/ } \\
\text { PVDF (10) }\end{array}$ & & $\mathrm{Li}$ & $\begin{array}{l}1 \mathrm{M} \mathrm{LiPF}_{6} \text { in } \\
\mathrm{EC} / \mathrm{DEC}\end{array}$ & 1.10 & $\begin{array}{l}557\left(1^{\text {st }} \text { cycle }\right) \text { vs. } 489 \\
\left(1000^{\text {th }} \text { cycle }\right) \text { at } 2500 \mathrm{~mA} \\
\mathrm{~g}^{-1}\end{array}$ & 12 \\
\hline 12 & & 1822 & $\begin{array}{l}12(70) / \text { CNT (20) / } \\
\text { PVDF (10) }\end{array}$ & & $\mathrm{Li}$ & $\begin{array}{l}1 \mathrm{M} \mathrm{LiPF}_{6} \text { in } \\
\mathrm{EC} / \mathrm{DEC}\end{array}$ & 1.15 & $\begin{array}{l}1000\left(1^{\text {st }} \text { cycle }\right) \text { vs. } 1500 \\
\left(100^{\text {th }} \text { cycle }\right) \text { at } 100 \mathrm{~mA} \\
\mathrm{~g}^{-1}\end{array}$ & 13 \\
\hline 13 & & & $\begin{array}{l}\mathbf{1 3}(60) \text { / SP (30) / PTFE } \\
(10)\end{array}$ & 3 & $\mathrm{Li}$ & $\begin{array}{l}1 \mathrm{M} \mathrm{LiPF}_{6} \text { in } \mathrm{EC} / \\
\mathrm{DEC} / \mathrm{DME}\end{array}$ & 0.60 & $\begin{array}{l}1042\left(1^{\text {st }} \text { cycle at } 20 \mathrm{~mA}\right. \\
\left.\mathrm{g}^{-1}\right) \text { vs. } 404\left(700^{\text {th }} \text { cycle at }\right. \\
\left.100 \mathrm{~mA} \mathrm{~g}^{-1}\right)\end{array}$ & 14 \\
\hline \multicolumn{10}{|c|}{ Carbonyl-based small molecules } \\
\hline 14 & & 256 & $\begin{array}{l}\mathbf{1 4}(60) / \mathrm{KB}(30) / \mathrm{PTFE} \\
(10)\end{array}$ & 2 & $\mathrm{Li}$ & $\begin{array}{l}1 \mathrm{M} \text { LiTFSI in } \\
\text { DOL/DME }\end{array}$ & 2.50 & $\begin{array}{l}119\left(1^{\text {st }} \text { cycle }\right) \text { vs. } 60\left(20^{\text {th }}\right. \\
\text { cycle }) \text { at } 50 \mathrm{~mA} \mathrm{~g}^{-1}\end{array}$ & 15 \\
\hline 15 & & 496 & 15/Graphite & & AHE & $2 \mathrm{M} \mathrm{H}_{2} \mathrm{SO}_{4}$ & & & 16 \\
\hline 16 & & 394 & $\begin{array}{l}\mathbf{1 6}(10) / \text { VGCF (80) / } \\
\text { PTFE (10) }\end{array}$ & & $\mathrm{Li}$ & $\begin{array}{l}2.75 \mathrm{M} \text { LiTFSI in } \\
\text { TEGDME }\end{array}$ & 2.50 & $\begin{array}{l}226\left(1^{\text {st }} \text { cycle }\right) \text { vs. } 129 \\
\left(15^{\text {th }} \text { cycle }\right) \text { at } 50 \mathrm{~mA} \mathrm{~g}^{-1}\end{array}$ & 17 \\
\hline
\end{tabular}


Table S2 (continued)

\begin{tabular}{|c|c|c|c|c|c|c|c|c|c|}
\hline Number & Structure & $\begin{array}{l}\text { Specific capacity } \\
\text { b) } / \mathrm{mAh} \mathrm{g}^{-1}\end{array}$ & Cathode composition ${ }^{c)}$ & $\begin{array}{l}\text { AM loading d) / } \\
\mathrm{mg} \mathrm{cm}^{-2}\end{array}$ & Anode & Electrolyte & $\begin{array}{l}\text { Voltage }{ }^{\mathrm{e})} / \mathrm{V} \\
\text { vs. } \mathrm{Li}^{+} \mathrm{Li}^{+}\end{array}$ & Cyclability / $\mathrm{mAh} \mathrm{g}^{-1}$ & Ref \\
\hline 17 & & 319 & $\begin{array}{l}\mathbf{1 7}(40) / \mathrm{AB}(50) / \mathrm{PTFE} \\
(10)\end{array}$ & 3 & $\mathrm{Li}$ & $\begin{array}{l}1 \mathrm{M} \mathrm{LiClO}_{4} \text { in } \gamma- \\
\text { butyl lactone }\end{array}$ & 2.65 & $\begin{array}{l}312\left(1^{\text {st }} \text { cycle }\right) \text { vs. } 255\left(10^{\text {th }}\right. \\
\text { cycle }) \text { at } 20 \mathrm{~mA} \mathrm{~g}^{-1}\end{array}$ & 18 \\
\hline 18 & & 501 & $\begin{array}{l}\mathbf{1 8}(5) / \text { VGCF (85) / } \\
\text { PTFE (10) }\end{array}$ & 3 & $\mathrm{Li}$ & $\begin{array}{l}1 \mathrm{M} \mathrm{LiPF}_{6} \text { in } \\
\mathrm{EC} / \mathrm{DEC}\end{array}$ & 2.90 & $\begin{array}{l}358\left(1^{\text {st }} \text { cycle }\right) \text { vs. } 170\left(20^{\text {th }}\right. \\
\text { cycle }) \text { at } 40 \mathrm{~mA} \mathrm{~g}^{-1}\end{array}$ & 18 \\
\hline 19 & & 409 & $\begin{array}{l}\mathbf{1 9}(56) / \mathrm{CMK}-3(24) / \\
\mathrm{CB}(10) / \mathrm{PVDF}(10)\end{array}$ & & $\mathrm{Li}$ & $\begin{array}{l}2 \mathrm{M} \mathrm{LiTFSI}^{+}+1 \% \\
\mathrm{LiNO}_{3} \text { in } \\
\mathrm{DOL} \mathrm{DME}\end{array}$ & 2.80 & $\begin{array}{l}404\left(\left(^{\text {st }} \text { cycle }\right) \text { vs. } 340\right. \\
\left(100^{\text {th }} \text { cycle }\right) \text { at } 0.2 \mathrm{C}\end{array}$ & 19 \\
\hline 20 & & 317 & $\begin{array}{l}20(56) / \text { CMK-3 (24)/ } \\
\text { CB (10) / PVDF (10) }\end{array}$ & & $\mathrm{Li}$ & $\begin{array}{l}2 \mathrm{M} \mathrm{LiTFSI}^{+}+1 \% \\
\mathrm{LiNO}_{3} \text { in } \\
\mathrm{DOL} / \mathrm{DME}\end{array}$ & 2.40 & $\begin{array}{l}310\left({ }^{\text {st }} \text { cycle }\right) \text { vs. } 220 \\
\left(100^{\text {th }} \text { cycle }\right) \text { at } 0.2 \mathrm{C}\end{array}$ & 19 \\
\hline 21 & & 327 & $\begin{array}{l}21 \text { (56) / CMK-3 (24)/ } \\
\text { CB (10) / PVDF (10) }\end{array}$ & & $\mathrm{Li}$ & $\begin{array}{l}2 \mathrm{M} \mathrm{LiTFSI}^{+}+1 \% \\
\mathrm{LiNO}_{3} \text { in } \\
\mathrm{DOL} / \mathrm{DME}^{-}\end{array}$ & 2.30 & $\begin{array}{l}322\left(1^{\text {st }} \text { cycle }\right) \text { vs. } 258 \\
\left(100^{\text {th }} \text { cycle }\right) \text { at } 0.2 \mathrm{C}\end{array}$ & 19 \\
\hline \multirow{2}{*}{22} & & & $\begin{array}{l}22(56) / \text { CMK-3 (24) / } \\
\text { CB (10) / PVDF (10) }\end{array}$ & & $\mathrm{Li}$ & $\begin{array}{l}2 \mathrm{M} \mathrm{LiTFSI}^{+}+1 \% \\
\mathrm{LiNO}_{3} \text { in } \\
\mathrm{DOL} \mathrm{DME}\end{array}$ & 2.25 & $\begin{array}{l}307\left(1^{\text {st }} \text { cycle }\right) \text { vs. } 246 \\
\left(100^{\text {th }} \text { cycle }\right) \text { at } 0.2 \mathrm{C}\end{array}$ & 19 \\
\hline & & & $\begin{array}{l}22(56) / \text { CMK-3 (24) / } \\
\text { CB (10) / PVDF (10) }\end{array}$ & & $\mathrm{Li}$ & $\begin{array}{l}1 \mathrm{M} \mathrm{LiPF}_{6} \text { in } \\
\mathrm{EC} / \mathrm{DMC}^{-}\end{array}$ & 2.25 & $\begin{array}{l}309\left(1^{\text {st }} \text { cycle }\right) \text { vs. } 203\left(50^{\text {th }}\right. \\
\text { cycle }) \text { at } 0.1 \mathrm{C}\end{array}$ & 20 \\
\hline 23 & & 220 & $\begin{array}{l}\mathbf{2 3}(5) / \text { VGCF (85) / } \\
\text { PTFE (10) }\end{array}$ & & $\mathrm{Li}$ & $\begin{array}{l}1 \mathrm{M} \mathrm{LiPF}_{6} \text { in } \\
\mathrm{EC} / \mathrm{DEC}\end{array}$ & 3.00 & $\begin{array}{l}162(1 \mathrm{st} \text { cycle }) \text { vs. } 60\left(20^{\text {th }}\right. \\
\text { cycle) at } 0.09 \mathrm{~mA} \mathrm{~cm}^{-2}\end{array}$ & 21 \\
\hline
\end{tabular}


Table S2 (continued)

\begin{tabular}{|c|c|c|c|c|c|c|c|c|c|}
\hline Number & Structure & $\begin{array}{l}\text { Specific capacity } \\
\text { b) } / \mathrm{mAh} \mathrm{g}^{-1}\end{array}$ & Cathode composition & $\begin{array}{l}\text { AM loading }{ }^{\text {b) } /} \\
\mathrm{mg} \mathrm{cm}^{-2}\end{array}$ & Anode & Electrolyte & $\begin{array}{l}\text { Voltage }^{\mathrm{c})} / \mathrm{V} \\
\text { vs. } \mathrm{Li}^{+} / \mathrm{Li}\end{array}$ & Cyclability / $\mathrm{mAh} \mathrm{g}^{-1}$ & Ref \\
\hline 24 & & 99 & $\begin{array}{l}\mathbf{2 4}(5) / \text { VGCF (85) / } \\
\text { PTFE (10) }\end{array}$ & & $\mathrm{Li}$ & $\begin{array}{l}1 \mathrm{M} \mathrm{LiPF}_{6} \text { in } \\
\mathrm{EC} / \mathrm{DEC}\end{array}$ & 3.00 & $\begin{array}{l}115\left(1^{\text {st }} \text { cycle }\right) \text { vs. } 58 \\
\left(20^{\text {th }} \text { cycle }\right) \text { at } 0.09 \mathrm{~mA} \\
\mathrm{~cm}^{-2}\end{array}$ & 21 \\
\hline 25 & & 66 & $\begin{array}{l}\mathbf{2 5}(5) / \text { VGCF (85) / } \\
\text { PTFE (10) }\end{array}$ & & $\mathrm{Li}$ & $\begin{array}{l}1 \mathrm{M} \mathrm{LiPF}_{6} \text { in } \\
\mathrm{EC} / \mathrm{DEC}\end{array}$ & 3.10 & $\begin{array}{l}177 \text { ( }\left(1^{\text {st }} \text { cycle }\right) \text { vs. } 98 \\
\left(20^{\text {th }} \text { cycle }\right) \text { at } 0.09 \mathrm{~mA} \\
\mathrm{~cm}^{-2}\end{array}$ & 21 \\
\hline 26 & & 257 & $\begin{array}{l}\mathbf{2 6}(30) / \mathrm{CC}(30) / \mathrm{AG} \\
(30) / \mathrm{PVDF}(10)\end{array}$ & 1.6 & $\mathrm{Li}$ & $\begin{array}{l}1 \mathrm{M} \mathrm{LiPF}_{6} \text { in } \\
\mathrm{EC} / \mathrm{DMC}^{-}\end{array}$ & 2.27 & $\begin{array}{l}210\left(1^{\text {st }} \text { cycle }\right) \text { vs. } 137 \\
\left(100^{\text {th }} \text { cycle }\right) \text { at } 0.1 \mathrm{C}\end{array}$ & 22 \\
\hline 27 & & 285 & $\begin{array}{l}27(30) / \mathrm{CC}(30) / \mathrm{AG} \\
(30) / \mathrm{PVDF}(10)\end{array}$ & 1.6 & $\mathrm{Li}$ & $\begin{array}{l}1 \mathrm{M} \mathrm{LiPF}_{6} \text { in } \\
\mathrm{EC} / \mathrm{DMC}\end{array}$ & 2.50 & $\begin{array}{l}220\left(1^{\text {st }} \text { cycle }\right) \text { vs. } 190 \\
\left(100^{\text {th }} \text { cycle }\right) \text { at } 0.1 \mathrm{C}\end{array}$ & 22 \\
\hline 28 & & 243 & $\begin{array}{l}\mathbf{2 8}(30) / \mathrm{CC}(30) / \mathrm{AG} \\
(30) / \mathrm{PVDF}(10)\end{array}$ & 1.6 & $\mathrm{Li}$ & $\begin{array}{l}1 \mathrm{M} \mathrm{LiPF}_{6} \text { in } \\
\mathrm{EC} / \mathrm{DMC}^{-}\end{array}$ & 2.52 & $\begin{array}{l}200\left(1^{\text {st }} \text { cycle }\right) \text { vs. } 108 \\
\left(100^{\text {th }} \text { cycle }\right) \text { at } 0.1 \mathrm{C}\end{array}$ & 22 \\
\hline 29 & & 255 & $\begin{array}{l}\mathbf{2 9}(30) / \mathrm{CC}(30) / \mathrm{AG} \\
(30) / \mathrm{PVDF}(10)\end{array}$ & 1.6 & $\mathrm{Li}$ & $\begin{array}{l}1 \mathrm{M} \mathrm{LiPF}_{6} \text { in } \\
\mathrm{EC} / \mathrm{DMC}^{-}\end{array}$ & 2.71 & $\begin{array}{l}190\left(1^{\text {st }} \text { cycle }\right) \text { vs. } 152 \\
\left(100^{\text {th }} \text { cycle }\right) \text { at } 0.1 \mathrm{C}\end{array}$ & 22 \\
\hline 30 & & 239 & $\begin{array}{l}\mathbf{3 0}(15) / \mathrm{AB}(40) / \\
\mathrm{PVDF}(100)\end{array}$ & & $\mathrm{Li}$ & $\begin{array}{l}1.25 \mathrm{M} \mathrm{LiPF}_{6} \text { in } \\
\mathrm{EC} / \mathrm{DEC}\end{array}$ & 2.30 & $\begin{array}{l}222\left(1^{\text {st }} \text { cycle }\right) \text { vs. } 75 \\
\left(40^{\text {th }} \text { cycle }\right) \text { at } 0.1 \mathrm{C}\end{array}$ & 23 \\
\hline
\end{tabular}


Table S2 (continued)

\begin{tabular}{|c|c|c|c|c|c|c|c|c|c|}
\hline Number & Structure & $\begin{array}{l}\text { Specific capacity } \\
\text { b) } / \mathrm{mAh} \mathrm{g}^{-1}\end{array}$ & Cathode composition $^{\mathrm{c})}$ & $\begin{array}{l}\text { AM loading d) / } \\
\mathrm{mg} \mathrm{cm}^{-2}\end{array}$ & Anode & Electrolyte & $\begin{array}{l}\text { Voltage }^{\mathrm{e})} / \mathrm{V} \\
\text { vs. } \mathrm{Li}^{\mathrm{L}} \mathrm{Li}^{+}\end{array}$ & Cyclability / $\mathrm{mAh} \mathrm{g}^{-1}$ & Ref \\
\hline 31 & & 257 & $\begin{array}{l}31(30) / \text { CB (50) / } \\
\text { PVDF (20) }\end{array}$ & 1.35 & $\mathrm{Li}$ & $\begin{array}{l}1 \mathrm{M} \mathrm{LiPF}_{6} \text { in } \\
\mathrm{EC} / \mathrm{DMC}\end{array}$ & 2.61 & $243\left(1^{\text {st }}\right.$ cycle $)$ & 24 \\
\hline 32 & & 255 & $\begin{array}{l}32(30) / \text { CB }(50) / \\
\text { PVDF (20) }\end{array}$ & 1.35 & $\mathrm{Li}$ & $\begin{array}{l}1 \mathrm{M} \mathrm{LiPF}_{6} \text { in } \\
\mathrm{EC} / \mathrm{DMC}\end{array}$ & 2.74 & $231\left(1^{\text {st }}\right.$ cycle $)$ & 24 \\
\hline 33 & & 243 & $\begin{array}{l}33(30) / \mathrm{CB}(50) / \\
\mathrm{PVDF}(20)\end{array}$ & 1.35 & $\mathrm{Li}$ & $\begin{array}{l}1 \mathrm{M} \mathrm{LiPF}_{6} \text { in } \\
\mathrm{EC} / \mathrm{DMC}^{-}\end{array}$ & 2.68 & $214\left(1^{\text {st }}\right.$ cycle $)$ & 24 \\
\hline 34 & & 200 & $\begin{array}{l}34(30) / \text { CB (50) / } \\
\text { PVDF (20) }\end{array}$ & 1.35 & $\mathrm{Li}$ & $\begin{array}{l}1 \mathrm{M} \mathrm{LiPF}_{6} \text { in } \\
\mathrm{EC} / \mathrm{DMC}\end{array}$ & 2.43 & $191\left(1^{\text {st }}\right.$ cycle $)$ & 24 \\
\hline 35 & & 409 & $\begin{array}{l}35(30) / \text { CB }(50) / \\
\text { PVDF (20) }\end{array}$ & 1.35 & $\mathrm{Li}$ & $\begin{array}{l}1 \mathrm{M} \mathrm{LiPF}_{6} \text { in } \\
\mathrm{EC} / \mathrm{DMC}\end{array}$ & 2.59 & $\begin{array}{l}360\left(1^{\text {st }} \text { cycle }\right) \text { vs. } 253 \\
\left(50^{\text {th }} \text { cycle }\right)\end{array}$ & 24 \\
\hline 36 & & 403 & $\begin{array}{l}\mathbf{3 6}(75) / \mathrm{AB}(15) / \\
\text { PVDF (10) }\end{array}$ & & $\mathrm{Li}$ & $\begin{array}{l}1 \mathrm{M} \mathrm{LiPF}_{6} \text { in } \\
\mathrm{DMC} / \mathrm{EMC} / \mathrm{EC}\end{array}$ & 2.77 & $243\left(1^{\text {st }}\right.$ cycle $)$ & 25 \\
\hline 37 & & 137 & $\begin{array}{l}37(80) / \mathrm{AB}(15) / \\
\text { PTFE (5) }\end{array}$ & & $\mathrm{Li}$ & $\begin{array}{l}1 \mathrm{M} \mathrm{LiClO}_{4} \text { in } \\
\mathrm{EC} / \mathrm{DMC}\end{array}$ & 2.40 & $\begin{array}{l}128\left(1^{\text {st }} \text { cycle }\right) \text { vs. } 64 \\
\left(90^{\text {th }} \text { cycle }\right) \text { at } 100 \mathrm{~mA} \\
\mathrm{~g}^{-1}\end{array}$ & 26 \\
\hline 38 & & 201 & $\begin{array}{l}38(45) / A B(50) / \\
\text { PVDF (5) }\end{array}$ & & $\mathrm{Li}$ & $\begin{array}{l}1 \mathrm{M} \mathrm{LiClO}_{4} \text { in } \\
\mathrm{EC} / \mathrm{DMC}\end{array}$ & 2.55 & $\begin{array}{l}121\left(1^{\text {st }} \text { cycle }\right) \text { vs. } 109 \\
\left(20^{\text {th }} \text { cycle }\right) \text { at } 0.2 \mathrm{C}\end{array}$ & 27 \\
\hline 39 & & 85 & $\begin{array}{l}39(60) \text { / CB (30) / } \\
\text { Kynar (10) }\end{array}$ & & $\mathrm{Li}$ & $\mathrm{LiPF}_{6}$ in $\mathrm{EC} / \mathrm{DMC}$ & 2.50 & $\begin{array}{l}107\left(1^{\text {st }} \text { cycle }\right) \text { vs. } 75 \\
\left(200^{\text {th }} \text { cycle }\right) \text { at } 5 \mathrm{C}\end{array}$ & 28 \\
\hline
\end{tabular}


Table S2 (continued)

\begin{tabular}{|c|c|c|c|c|c|c|c|c|c|}
\hline Number & Structure & $\begin{array}{l}\text { Specific capacity } \\
\text { b) } / \mathrm{mAh} \mathrm{g}^{-1}\end{array}$ & Cathode composition ${ }^{\mathrm{c})}$ & $\begin{array}{l}\text { AM loading d) / } \\
\mathrm{mg} \mathrm{cm}^{-2}\end{array}$ & Anode & Electrolyte & $\begin{array}{l}\text { Voltage }^{\mathrm{e})} / \mathrm{V} \\
\text { vs. } \mathrm{Li}^{\mathrm{L}} \mathrm{Li}^{+}\end{array}$ & Cyclability / $\mathrm{mAh} \mathrm{g}^{-1}$ & Ref \\
\hline 40 & & 317 & $\begin{array}{l}\mathbf{4 0}(40) / \text { CB }(50) / \\
\text { PTFE }(10)\end{array}$ & 6 & $\mathrm{Li}$ & LiTFSI in TG & 2.00 & $\begin{array}{l}236\left(1^{\text {st }} \text { cycle }\right) \text { vs. } 183 \\
\left(100^{\text {th }} \text { cycle }\right) \text { at } 20 \mathrm{~mA} \mathrm{~g}^{-1}\end{array}$ & 29 \\
\hline 41 & & 628 & $\begin{array}{l}41(50) / A B(45) / \\
\text { PVDF (5) }\end{array}$ & 1 & $\mathrm{Li}$ & $\begin{array}{l}1 \mathrm{M} \mathrm{LiPF}_{6} \text { in } \\
\mathrm{EC} / \mathrm{DEC}\end{array}$ & 2.70 & $\begin{array}{l}340\left(1^{\text {st }} \text { cycle }\right) \text { vs. } 201\left(40^{\text {th }}\right. \\
\text { cycle }) \text { at } 200 \mathrm{~mA} \mathrm{~g}^{-1}\end{array}$ & 30 \\
\hline 42 & & 282 & 42 & $2.0-3.0$ & $\mathrm{Li}$ & $\begin{array}{l}1 \mathrm{M} \text { LiTFSI in } \\
\mathrm{DOL} / \mathrm{DME}\end{array}$ & 2.30 & $\begin{array}{l}280\left(1^{\text {st }} \text { cycle }\right) \text { vs. } 277 \\
\left(1000^{\text {th }} \text { cycle }\right) \text { at } 0.5 \mathrm{C}\end{array}$ & 31 \\
\hline 43 & & 289.9 & $\begin{array}{l}\mathbf{4 3}(60) \text { / SP (30) / } \\
\text { PVDF (10) }\end{array}$ & $1.8-2.5$ & $\mathrm{Li}$ & $\begin{array}{l}1 \text { M LiTFSI in } \\
\text { DME/DIOX }\end{array}$ & 2.25 & $\begin{array}{l}250\left(1^{\text {st }} \text { cycle }\right) \text { vs. } 248 \\
\left(500^{\text {th }} \text { cycle }\right) \text { at } 0.2 \mathrm{C}\end{array}$ & 32 \\
\hline 44 & & 233 & $\begin{array}{l}\mathbf{4 4}(50) / \text { CB (40) / } \\
\text { PVDF (10) }\end{array}$ & $1-1.4$ & $\mathrm{Li}$ & $\begin{array}{l}1 \mathrm{M} \text { LiTFSI in } \\
\mathrm{DOL} / \mathrm{DME}\end{array}$ & 2.40 & $\begin{array}{l}222\left(1^{\text {st }} \text { cycle }\right) \text { vs. } 203 \\
\left(100^{\text {th }} \text { cycle }\right) \text { at } 0.2 \mathrm{C}\end{array}$ & 33 \\
\hline 45 & & 243 & $\begin{array}{l}45(60) / \text { KB }(30) / \\
\text { PTFE }(10)\end{array}$ & 2 & $\mathrm{Li}$ & $\begin{array}{l}1 \mathrm{M} \text { LiTFSI in } \\
\text { DOL/DME }\end{array}$ & 2.10 & $\begin{array}{l}193\left(1^{\text {st }} \text { cycle }\right) \text { vs. } 145\left(20^{\text {th }}\right. \\
\text { cycle }) \text { at } 50 \mathrm{~mA} \mathrm{~g}^{-1}\end{array}$ & 15 \\
\hline 46 & & 590 & $\begin{array}{l}\mathbf{4 6}(60) / \mathrm{SP}(30) / \\
\mathrm{PVDF}(10)\end{array}$ & & $\mathrm{Li}$ & $\begin{array}{l}1 \mathrm{M} \mathrm{LiPF}_{6} \text { in } \\
\mathrm{EC} / \mathrm{DMC}^{-}\end{array}$ & 2.40 & $\begin{array}{l}580\left(1^{\text {st }} \text { cycle }\right) \text { vs. } 116\left(20^{\text {th }}\right. \\
\text { cycle }) \text { at } 50 \mathrm{~mA} \mathrm{~g}^{-1}\end{array}$ & 34 \\
\hline 47 & & 353 & $\begin{array}{l}47(60) / \mathrm{AB}(30) / \\
\mathrm{PTFE}(10)\end{array}$ & 4.63 & $\mathrm{Li}$ & $\begin{array}{l}1 \mathrm{M} \mathrm{LiPF}_{6} \text { in } \\
\mathrm{EC} / \mathrm{DMC}^{-}\end{array}$ & 1.70 & $\begin{array}{l}176\left(1^{\text {st }} \text { cycle }\right) \text { vs. } 137\left(10^{\text {th }}\right. \\
\text { cycle }) \text { at } 100 \mathrm{~mA} \mathrm{~g}^{-1}\end{array}$ & 35 \\
\hline
\end{tabular}


Table S2 (continued)

\begin{tabular}{|c|c|c|c|c|c|c|c|c|c|}
\hline Number & Structure & $\begin{array}{l}\text { Specific capacity } \\
\text { b) } / \mathrm{mAh} \mathrm{g}^{-1}\end{array}$ & Cathode composition ${ }^{c)}$ & $\begin{array}{l}\text { AM loading d) / } \\
\mathrm{mg} \mathrm{cm}^{-2}\end{array}$ & Anode & Electrolyte & $\begin{array}{l}\text { Voltage }^{\mathrm{e})} / \mathrm{V} \\
\text { vs. } \mathrm{Li}^{\mathrm{L}} \mathrm{Li}^{+}\end{array}$ & Cyclability / $\mathrm{mAh} \mathrm{g}^{-1}$ & Ref \\
\hline 48 & & 590 & $48(85)$ / KB (15) & $10-12 \mathrm{mg}$ & $\mathrm{Li}$ & $\begin{array}{l}1 \mathrm{M} \mathrm{LiPF}_{6} \text { in } \\
\mathrm{EC} / \mathrm{DMC}\end{array}$ & 2.40 & $\begin{array}{l}590\left(1^{\text {st }} \text { cycle }\right) \text { vs. } 250\left(14^{\text {th }}\right. \\
\text { cycle }) \text { at } 1 / 40 \mathrm{C}\end{array}$ & 36 \\
\hline 49 & & 288 & $\begin{array}{l}49(70) \text { / CB (20) / PVDF } \\
(10)\end{array}$ & 0.8 & $\mathrm{Li}$ & $\begin{array}{l}1 \mathrm{M} \mathrm{LiPF}_{6} \text { in } \\
\mathrm{EC} / \mathrm{DMC}\end{array}$ & 1.60 & $\begin{array}{l}177\left(1^{\text {st }} \text { cycle }\right) \text { vs. } 177 \\
\left(100^{\text {th }} \text { cycle }\right) \text { at } 0.2 \mathrm{C}\end{array}$ & 37 \\
\hline 50 & & 241 & $\mathbf{5 0}(50) / \mathrm{KB}(50)$ & & $\mathrm{Li}$ & $\begin{array}{l}1 \mathrm{M} \mathrm{LiPF}_{6} \text { in } \\
\mathrm{EC} / \mathrm{DMC}\end{array}$ & 2.80 & $\begin{array}{l}105\left(1^{\text {st }} \text { cycle }\right) \text { vs. } 105\left(30^{\text {th }}\right. \\
\text { cycle }) \text { at } 0.1 \mathrm{C}\end{array}$ & 38 \\
\hline 51 & & 174 & $\begin{array}{l}\mathbf{5 1}(15) / \mathrm{AB}(40) / \mathrm{PTFE} \\
(10)\end{array}$ & 4.2 & $\mathrm{Li}$ & $1 \mathrm{M} \mathrm{LiPF}_{6}$ in $\mathrm{PC}$ & 1.79 & $\begin{array}{l}85\left(1^{\text {st }} \text { cycle }\right) \text { vs. } 70\left(20^{\text {th }}\right. \\
\text { cycle }) \text { at } 0.2 \mathrm{C}\end{array}$ & 39 \\
\hline 52 & & 174 & $\begin{array}{l}\mathbf{5 2}(15) / \mathrm{AB}(40) / \mathrm{PTFE} \\
(10)\end{array}$ & 4.2 & $\mathrm{Li}$ & $1 \mathrm{M} \mathrm{LiPF}_{6}$ in $\mathrm{PC}$ & 2.11 & $\begin{array}{l}90\left(1^{\text {st }} \text { cycle }\right) \text { vs. } 95\left(2^{\text {nd }}\right. \\
\text { cycle }) \text { at } 0.2 \mathrm{C}\end{array}$ & 39 \\
\hline 53 & & 296 & $\begin{array}{l}\mathbf{5 3}(15) / \mathrm{AB}(40) / \mathrm{PTFE} \\
(10)\end{array}$ & 4.2 & $\mathrm{Li}$ & $1 \mathrm{M} \mathrm{LiPF}_{6}$ in $\mathrm{PC}$ & 2.39 & $\begin{array}{l}217\left(1^{\text {st }} \text { cycle }\right) \text { vs. } 187\left(20^{\text {th }}\right. \\
\text { cycle }) \text { at } 0.2 \mathrm{C}\end{array}$ & 39 \\
\hline 54 & & 212 & $\begin{array}{l}\mathbf{5 4}(60) / \mathrm{AB}(30) / \mathrm{PTFE} \\
(10)\end{array}$ & & $\mathrm{Li}$ & $\begin{array}{l}1 \mathrm{M} \mathrm{LiPF}_{6} \text { in } \mathrm{EC} / \\
\mathrm{EMC} / \mathrm{DMC}\end{array}$ & 1.60 & $\begin{array}{l}115\left(1^{\text {st }} \text { cycle }\right) \text { vs. } 115 \\
\left(50^{\text {th }} \text { cycle }\right) \text { at } 111 \mathrm{~mA} \mathrm{~g}^{-1}\end{array}$ & 40 \\
\hline 55 & & 130 & $\begin{array}{l}\mathbf{5 5}(70) / \mathrm{AB}(20) / \mathrm{PVDF} \\
(10)\end{array}$ & 2 & $\mathrm{Li}$ & $1 \mathrm{M} \mathrm{LiPF}_{6}$ in $\mathrm{DMC}$ & 2.41 & $\begin{array}{l}130\left(1^{\text {st }} \text { cycle }\right) \text { vs. } 120 \\
\left(100^{\text {th }} \text { cycle }\right) \text { at } 0.1 \mathrm{C}\end{array}$ & 41 \\
\hline 56 & & 236 & 56 (67) / SP (33) & & $\mathrm{Li}$ & $\begin{array}{l}1 \mathrm{M} \text { LiTFSI in } \\
\text { DMC }\end{array}$ & 1.70 & $\begin{array}{l}200\left(1^{\text {st }} \text { cycle }\right) \text { vs. } 200\left(20^{\text {th }}\right. \\
\text { cycle }) \text { at } 1 / 40 \mathrm{C}\end{array}$ & 42 \\
\hline
\end{tabular}


Table S2 (continued)

\begin{tabular}{|c|c|c|c|c|c|c|c|c|c|}
\hline Number & Structure & $\begin{array}{l}\text { Specific capacity } \\
\text { b) } / \mathrm{mAh} \mathrm{g}^{-1}\end{array}$ & Cathode composition ${ }^{c)}$ & $\begin{array}{l}\text { AM loading d) / } \\
\mathrm{mg} \mathrm{cm}^{-2}\end{array}$ & Anode & Electrolyte & $\begin{array}{l}\text { Voltage }^{\mathrm{e})} / \mathrm{V} \\
\text { vs. } \mathrm{Li} / \mathrm{Li}^{+}\end{array}$ & Cyclability / $\mathrm{mAh} \mathrm{g}^{-1}$ & $\operatorname{Re}$ \\
\hline 57 & & & $\begin{array}{l}\mathbf{5 7}(60) / \mathrm{CB}(30) / \mathrm{SA} \\
(10)\end{array}$ & 1.5 & $\mathrm{Li}$ & $\begin{array}{l}7 \mathrm{M} \text { LiTFSI in } \\
\text { DOL/DME }\end{array}$ & 1.90 & $\begin{array}{l}153\left(1^{\text {st }} \text { cycle }\right) \text { vs. } 131 \\
\left(100^{\text {th }} \text { cycle }\right) \text { at } 0.5 \mathrm{C}\end{array}$ & 43 \\
\hline 58 & & 330 & $\mathbf{5 8}(70) / \mathrm{CB}(30)$ & $7-8.4$ & $\mathrm{Li}$ & $\begin{array}{l}1 \mathrm{M} \mathrm{LiPF}_{6} \text { in } \\
\mathrm{EC} / \mathrm{DMC}^{-}\end{array}$ & 0.80 & $\begin{array}{l}300\left(1^{\text {st }} \text { cycle }\right) \text { vs. } 234\left(50^{\text {th }}\right. \\
\text { cycle }) \text { at } 1 / 20 \mathrm{C}\end{array}$ & 44 \\
\hline 59 & & 263 & $\begin{array}{l}\mathbf{5 9}(60) / \mathrm{CB}(30) / \text { PVDF } \\
(10)\end{array}$ & & $\mathrm{Li}$ & $\begin{array}{l}1 \mathrm{M} \mathrm{LiPF}_{6} \text { in } \\
\mathrm{EC} / \mathrm{DMC}\end{array}$ & 0.80 & $\begin{array}{l}200\left(1^{\text {st }} \text { cycle }\right) \text { vs. } 155\left(50^{\text {th }}\right. \\
\text { cycle }) \text { at } 13.7 \mathrm{~mA} \mathrm{~g}^{-1}\end{array}$ & 45 \\
\hline 60 & & 141 & $\begin{array}{l}\mathbf{6 0}(60) / \mathrm{CB}(30) / \mathrm{PVDF} \\
(10)\end{array}$ & & $\mathrm{Li}$ & $\begin{array}{l}1 \mathrm{M} \mathrm{LiPF}_{6} \text { in } \\
\mathrm{EC} / \mathrm{DMC} / \mathrm{DEC}\end{array}$ & 0.73 & $\begin{array}{l}227\left(1^{\text {st }} \text { cycle }\right) \text { vs. } 114 \\
\left(500^{\text {th }} \text { cycle }\right) \text { at } 1 \mathrm{C}\end{array}$ & 46 \\
\hline 61 & & & $\begin{array}{l}\mathbf{6 1}(60) / \mathrm{CB}(30) / \mathrm{SA} \\
(10)\end{array}$ & 1.5 & $\mathrm{Li}$ & $\begin{array}{l}7 \mathrm{M} \text { LiTFSI in } \\
\mathrm{DOL} / \mathrm{DME}\end{array}$ & 1.30 & $\begin{array}{l}220\left(\left(1^{\text {st }} \text { cycle at } 0.5 \mathrm{C}\right) \text { vs. }\right. \\
95\left(500^{\text {th }} \text { cycle at } 10 \mathrm{C}\right)\end{array}$ & 43 \\
\hline 62 & & 349.1 & $\begin{array}{l}\mathbf{6 2}(40) / \mathrm{SP}(30) / \mathrm{CMC} \\
(10)\end{array}$ & & $\mathrm{Li}$ & $\begin{array}{l}0.8 \mathrm{M} \mathrm{LiPF}_{6} \text { in } \\
\mathrm{EC} / \mathrm{DEC}\end{array}$ & 1.35 & $\begin{array}{l}241\left(1^{\text {st }} \text { cycle }\right) \text { vs. } 239\left(50^{\text {th }}\right. \\
\text { cycle }) \text { at } 50 \mathrm{~mA} \mathrm{~g}^{-1}\end{array}$ & 47 \\
\hline 63 & & 234 & $\mathbf{6 3}(60)$ / SP (40) & & $\mathrm{Li}$ & $\begin{array}{l}1 \mathrm{M} \mathrm{LiPF}_{6} \text { in } \\
\mathrm{EC} / \mathrm{DMC}^{-}\end{array}$ & 1.10 & $\begin{array}{l}222\left(1^{\text {st }} \text { cycle }\right) \text { vs. } 125 \\
\left(100^{\text {th }} \text { cycle }\right) \text { at } 1.25 \mathrm{C}\end{array}$ & 48 \\
\hline \multicolumn{10}{|c|}{ Carbonyl-based polymers } \\
\hline \multirow[t]{2}{*}{64} & & 225 & $\begin{array}{l}\mathbf{6 4}(40) / \text { AB (40) / PTFE } \\
(20)\end{array}$ & & $\mathrm{Li}$ & $\begin{array}{l}1 \mathrm{M} \text { LiTFSI in } \\
\text { DOL/DME }\end{array}$ & 2.30 & $\begin{array}{l}198\left(1^{\text {st }} \text { cycle }\right) \text { vs. } 178 \\
\left(200^{\text {th }} \text { cycle }\right) \text { at } 50 \mathrm{~mA} \mathrm{~g}^{-1}\end{array}$ & 49 \\
\hline & & & $\begin{array}{l}\mathbf{6 4}(56.16) / \text { FGS }(3.84) / \\
\mathrm{CC}(30) / \mathrm{PTFE}(10)\end{array}$ & & $\mathrm{Li}$ & $\begin{array}{l}1 \mathrm{M} \text { LiTFSI in } \\
\text { DOL/DME }\end{array}$ & 2.20 & $\begin{array}{l}187\left(1^{\text {st }} \text { cycle }\right) \text { vs. } \sim 176 \\
\left(100^{\text {th }} \text { cycle }\right) \text { at } 0.1 \mathrm{C}\end{array}$ & 50 \\
\hline 65 & & 225 & $\begin{array}{l}\mathbf{6 5}(40) / \mathrm{SP}(40) / \mathrm{PVDF} \\
(20)\end{array}$ & & $\mathrm{Li}$ & $\begin{array}{l}1 \mathrm{M} \text { LiTFSI in } \\
\text { DOL/DME }\end{array}$ & 2.22 & $\begin{array}{l}90\left(1^{\text {st }} \text { cycle at } 0.1 \mathrm{C}\right) \text { vs. } \\
\sim 100\left(200^{\text {th }} \text { cycle at } 0.5 \mathrm{C}\right)\end{array}$ & 51 \\
\hline
\end{tabular}


Table S2 (continued)

\begin{tabular}{|c|c|c|c|c|c|c|c|c|c|}
\hline Number & Structure & $\begin{array}{l}\text { Specific capacity } \\
\text { b) } / \mathrm{mAh} \mathrm{g}^{-1}\end{array}$ & Cathode composition ${ }^{c)}$ & $\begin{array}{l}\text { AM loading d)/ } \\
\mathrm{mg} \mathrm{cm}^{-2}\end{array}$ & Anode & Electrolyte & $\begin{array}{l}\text { Voltage }^{\mathrm{e})} / \mathrm{V} \\
\text { vs. } \mathrm{Li}^{+} \mathrm{Li}^{+}\end{array}$ & Cyclability / $\mathrm{mAh} \mathrm{g}^{-1}$ & Ref \\
\hline 66 & & 260 & $\begin{array}{l}\mathbf{6 6}(60) / \mathrm{KB}(30) / \text { PTFE } \\
(10)\end{array}$ & $1-2$ & $\mathrm{Li}$ & $\begin{array}{l}1 \mathrm{M} \text { LiTFSI in } \\
\text { DOL/DME }\end{array}$ & 2.02 & $\begin{array}{l}235\left(1^{\text {st }} \text { cycle }\right) \text { vs. } 234 \\
\left(1000^{\text {th }} \text { cycle }\right) \text { at } 2 \mathrm{C}\end{array}$ & 52 \\
\hline 67 & & 260 & $\begin{array}{l}\mathbf{6 7}(60) / \mathrm{KB}(30) / \mathrm{PTFE} \\
(10)\end{array}$ & $1-2$ & $\mathrm{Li}$ & $\begin{array}{l}1 \mathrm{M} \text { LiTFSI in } \\
\text { DOL/DME }\end{array}$ & 1.85 & $\begin{array}{l}\sim 260\left(\sim 20^{\text {th }} \text { cycle }\right) \text { vs. } \sim 256 \\
\left(100^{\text {th }} \text { cycle }\right) \text { at } 0.2 \mathrm{C}\end{array}$ & 52 \\
\hline 68 & & 388 & $\begin{array}{l}\mathbf{6 8}(60) / \mathrm{KB}(30) / \mathrm{PTFE} \\
(10)\end{array}$ & $1-2$ & $\mathrm{Li}$ & $\begin{array}{l}1 \mathrm{M} \text { LiTFSI in } \\
\text { DOL/DME }\end{array}$ & 2.67 & $\begin{array}{l}275\left(1^{\text {st }} \text { cycle at } 50 \mathrm{~mA} \mathrm{~g}^{-1}\right) \\
\text { vs. } 212\left(1000^{\text {th }} \text { cycle at }\right. \\
\left.500 \mathrm{~mA} \mathrm{~g}^{-1}\right)\end{array}$ & 53 \\
\hline 69 & & 388 & $\begin{array}{l}\mathbf{6 9}(50) / \mathrm{CB}(35) / \mathrm{PTFE} \\
(15)\end{array}$ & & $\mathrm{Li}$ & $\begin{array}{l}1 \mathrm{M} \text { LiTFSI in } \\
\text { DOL/DME }\end{array}$ & 2.60 & $\begin{array}{l}\sim 240\left(20^{\text {th }} \text { cycle }\right) \text { vs. } 160 \\
\left(340^{\text {th }} \text { cycle }\right) \text { at } 50 \mathrm{~mA} \mathrm{~g}^{-1}\end{array}$ & 54 \\
\hline 70 & & 315 & $\begin{array}{l}70(60) / A B(30) / P V D F \\
(10)\end{array}$ & & $\mathrm{Li}$ & $\begin{array}{l}1 \mathrm{MLiPF}_{6} \text { in } \\
\mathrm{EC} / \mathrm{DMC}\end{array}$ & 2.20 & $\begin{array}{l}350\left(2^{\text {nd }} \text { cycle }\right) \text { vs. } 184 \\
\left(100^{\text {th }} \text { cycle }\right) \text { at } 15 \mathrm{~mA} \mathrm{~g}^{-1}\end{array}$ & 55 \\
\hline 71 & & 295 & $71(60) /(\mathrm{CC}) /(\mathrm{PTFE})$ & $1-2$ & $\mathrm{Li}$ & $\begin{array}{l}1 \mathrm{M} \text { LiTFSI in } \\
\text { DOL/DME }\end{array}$ & 2.10 & $\begin{array}{l}239\left(2^{\text {nd }} \text { cycle }\right) \text { vs. } 215 \\
\left(1500^{\text {th }} \text { cycle }\right) \text { at } 1.75 \mathrm{C}\end{array}$ & 15 \\
\hline \multirow{2}{*}{72} & & \multirow{2}{*}{352} & $\begin{array}{l}72(35) / \mathrm{AB}(60) / \mathrm{PTFE} \\
(5)\end{array}$ & 12.7 & $\mathrm{Li}$ & $\begin{array}{l}1 \mathrm{M} \mathrm{LiPF}_{6} \text { in } \\
\mathrm{EC} / \mathrm{DMC}^{-}\end{array}$ & 2.25 & $\begin{array}{l}150\left(1^{\text {st }} \text { cycle }\right) \text { vs. } 135 \\
\left(100^{\text {th }} \text { cycle }\right) \text { at } 0.5 \mathrm{C}\end{array}$ & 56 \\
\hline & & & $\begin{array}{l}72(60) / \text { PEDOT (30) / } \\
\text { PVDF (10) }\end{array}$ & & $\mathrm{Li}$ & $\begin{array}{l}1 \mathrm{M} \mathrm{LiPF}_{6} \text { in } \\
\mathrm{EC} / \mathrm{DMC}\end{array}$ & 2.20 & $140\left(1^{\text {st }}\right.$ cycle $)$ at $0.05 \mathrm{C}$ & 57 \\
\hline 73 & & 250 & $\begin{array}{l}73 \text { (41) / SP (34) / } \\
\text { Trilene-65 (25) }\end{array}$ & & $\mathrm{Li}$ & $\begin{array}{l}1 \mathrm{M} \mathrm{LiPF}_{6} \text { in } \\
\mathrm{EC} / \mathrm{DMC}^{-}\end{array}$ & 2.50 & $\begin{array}{l}60\left(1^{\text {st }} \text { cycle }\right) \text { vs. } 50\left(7^{\text {th }}\right. \\
\text { cycle) at } 0.04 \mathrm{C}\end{array}$ & 58 \\
\hline
\end{tabular}


Table S2 (continued)

\begin{tabular}{|c|c|c|c|c|c|c|c|c|c|}
\hline Number & Structure & $\begin{array}{l}\text { Specific capacity } \\
\text { b) } / \mathrm{mAh} \mathrm{g}^{-1}\end{array}$ & Cathode composition $^{\mathrm{c}}$ & $\begin{array}{l}\text { AM loading d)/ } \\
\mathrm{mg} \mathrm{cm}^{-2}\end{array}$ & Anode & Electrolyte & $\begin{array}{l}\text { Voltage }^{\mathrm{e})} / \mathrm{V} \\
\text { vs. } \mathrm{Li}^{+} \mathrm{Li}^{+}\end{array}$ & Cyclability / $\mathrm{mAh} \mathrm{g}^{-1}$ & $\mathrm{Re}$ \\
\hline 74 & & 250 & $\begin{array}{l}74(50) / A B(40) / \\
\text { PVDF (10) }\end{array}$ & & $\mathrm{Li}$ & $\begin{array}{l}1 \mathrm{M} \mathrm{LiPF}_{6} \text { in } \\
\mathrm{EC} / \mathrm{DEC}\end{array}$ & 2.25 & $\begin{array}{l}101\left(1^{\text {st }} \text { cycle }\right) \text { vs. } 129\left(50^{\text {th }}\right. \\
\text { cycle) at } 400 \mathrm{~mA} \mathrm{~g}^{-1}\end{array}$ & 59 \\
\hline 75 & & 217 & $\begin{array}{l}\mathbf{7 5}(10) \text { / PVDF (80) / } \\
\text { MWCNT (10) }\end{array}$ & & $\mathrm{Li}$ & $\begin{array}{l}1 \mathrm{M} \mathrm{LiClO}_{4} \text { in } \\
\mathrm{EC} / \mathrm{DMC}\end{array}$ & 2.23 & $\begin{array}{l}219\left(1^{\text {st }} \text { cycle }\right) \text { vs. } 116 \\
\left(100^{\text {th }} \text { cycle }\right) \text { at } 1 \mathrm{C}\end{array}$ & 60 \\
\hline 76 & & & 76 (53) / FWCNT (47) & & $\mathrm{Li}$ & $\begin{array}{l}1 \mathrm{M} \mathrm{LiPF}_{6} \text { in } \\
\mathrm{EC} / \mathrm{DMC}^{-}\end{array}$ & 2.50 & $\begin{array}{l}105\left(1^{\text {st }} \text { cycle }\right) \text { vs. } 115 \\
\left(10000^{\text {th }} \text { cycle }\right) \text { at } 100 \mathrm{~mA} \\
\mathrm{~g}^{-1}\end{array}$ & 61 \\
\hline 77 & & 146 & $\begin{array}{l}77 \text { (10) / VGCF (80) / } \\
\text { PVDF (10) }\end{array}$ & & $\mathrm{Ag} / \mathrm{AgCl}$ & $\begin{array}{l}0.1 \mathrm{M} \mathrm{TBAClO} \\
\text { in } \\
\text { ACN }\end{array}$ & 2.36 & $\begin{array}{l}62\left(1^{\text {st }} \text { cycle }\right) \text { vs. } 47\left(50^{\text {th }}\right. \\
\text { cycle })\end{array}$ & 62 \\
\hline 78 & & 262 & $\begin{array}{l}78(15) / \mathrm{AB}(40) / \\
\mathrm{PVDF}(10)\end{array}$ & 5.7 & $\mathrm{Li}$ & LiTFSI in $\mathrm{G}_{4}$ & 2.50 & $\begin{array}{l}231\left(1^{\text {st }} \text { cycle }\right) \text { vs. } 193 \\
\left(500^{\text {th }} \text { cycle }\right) \text { at } 1 \mathrm{C}\end{array}$ & 63 \\
\hline 79 & & 384 & $\begin{array}{l}79(60) / \mathrm{AB}(35) / \\
\operatorname{PTEE}(5)\end{array}$ & 4.63 & $\mathrm{Li}$ & $\begin{array}{l}1 \mathrm{M} \mathrm{LiPF}_{6} \text { in } \\
\mathrm{EC} / \mathrm{DMC}\end{array}$ & 1.90 & $\begin{array}{l}176\left(1^{\text {st }} \text { cycle }\right) \text { vs. } 137\left(10^{\text {th }}\right. \\
\text { cycle }) \text { at } 100 \mathrm{~mA} \mathrm{~g}^{-1}\end{array}$ & 64 \\
\hline 80 & & & $\begin{array}{l}\mathbf{8 0}(5) / \text { VGCF (85) / } \\
\text { PVDF (10) }\end{array}$ & & $\mathrm{Ag} / \mathrm{AgCl}$ & $\begin{array}{l}0.1 \mathrm{M} \mathrm{TBAClO} \\
\text { in } \\
\text { ACN }\end{array}$ & 1.90 & $\begin{array}{l}78\left(1^{\text {st }} \text { cycle }\right) \text { vs. } 55\left(10^{\text {th }}\right. \\
\text { cycle }) \text { at } 0.04 \mathrm{C}\end{array}$ & 65 \\
\hline
\end{tabular}


Table S2 (continued)

\begin{tabular}{|c|c|c|c|c|c|c|c|c|c|}
\hline Number & Structure & $\begin{array}{l}\text { Specific capacity } \\
\text { b) } / \mathrm{mAh} \mathrm{g}^{-1}\end{array}$ & Cathode composition $^{c)}$ & $\begin{array}{l}\text { AM loading d) / } \\
\mathrm{mg} \mathrm{cm}^{-2}\end{array}$ & Anode & Electrolyte & $\begin{array}{l}\text { Voltage }^{\mathrm{e})} / \mathrm{V} \\
\text { vs. Li/ } \mathrm{Li}^{+}\end{array}$ & Cyclability / $\mathrm{mAh} \mathrm{g}^{-1}$ & Ref \\
\hline 81 & & 185 & $\begin{array}{l}\mathbf{8 1}(5) \text { / VGCF (85) / } \\
\operatorname{PVDF~(10)~}\end{array}$ & & $\mathrm{Ag} / \mathrm{AgCl}$ & $\begin{array}{l}0.1 \mathrm{M} \mathrm{TBAClO} \\
\text { ACN }\end{array}$ & 2.50 & $\begin{array}{l}95\left(1^{\text {st }} \text { cycle) vs. } 71\left(10^{\text {th }}\right.\right. \\
\text { cycle) at } 0.03 \mathrm{C}\end{array}$ & 65 \\
\hline 82 & & 315 & $\begin{array}{l}\mathbf{8 2}(60) / \text { CC (30) / } \\
\text { PTFE (10) }\end{array}$ & & $\mathrm{Li}$ & $\begin{array}{l}1 \mathrm{M} \text { LiTFSI in } \\
\text { DOL/DME }\end{array}$ & 2.40 & $\begin{array}{l}163\left(1^{\text {st }} \text { cycle }\right) \text { vs. } 155\left(50^{\text {th }}\right. \\
\text { cycle) at } 0.2 \mathrm{C}\end{array}$ & 66 \\
\hline 83 & & 442.7 & 83 / SWCNT & & $\mathrm{Li}$ & $\begin{array}{l}1 \mathrm{M} \text { LiTFSI in } \\
\text { DOL/DME }\end{array}$ & 2.10 & $\begin{array}{l}206\left(1^{\text {st }} \text { cycle }\right) \text { vs. } 175 \\
\left(200^{\text {th }} \text { cycle }\right) \text { at } 0.5 \mathrm{C}\end{array}$ & 67 \\
\hline 84 & & 191 & 84 (90) / SWCNT (10) & 4 & $\mathrm{Li}$ & $\begin{array}{l}1 \mathrm{M} \text { LiTFSI in } \\
\text { DOL/DME }\end{array}$ & 2.00 & $\begin{array}{l}226\left(1^{\text {st }} \text { cycle at } 0.1 \mathrm{C}\right) \text { vs. } \\
192\left(200^{\text {th }} \text { cycle at } 0.5 \mathrm{C}\right)\end{array}$ & 68 \\
\hline 85 & & 250 & 85 (85) / KB (15) & 4.42 & $\mathrm{Li}$ & $\begin{array}{l}1 \mathrm{M} \text { LiTFSI in Me- } \\
\text { THF }\end{array}$ & 2.20 & $\begin{array}{l}125\left(1^{\text {st }} \text { cycle }\right) \text { vs. } 26\left(100^{\text {th }}\right. \\
\text { cycle }) \text { at } 0.1 \mathrm{C}\end{array}$ & 69 \\
\hline 86 & & 200 & $86(85)$ / KB (15) & 4.42 & $\mathrm{Li}$ & $\begin{array}{l}1 \mathrm{M} \text { LiTFSI in Me- } \\
\text { THF }\end{array}$ & 2.20 & $\begin{array}{l}196\left(1^{\text {st }} \text { cycle }\right) \text { vs. } 106 \\
\left(100^{\text {th }} \text { cycle }\right) \text { at } 0.1 \mathrm{C}\end{array}$ & 69 \\
\hline 87 & & 144 & $\begin{array}{l}87(35) / \mathrm{CB}(50) / \\
\text { PVDF (15) }\end{array}$ & & $\mathrm{Li}$ & $\begin{array}{l}1 \mathrm{M} \mathrm{LiPF}_{6} \text { in } \\
\text { TEGDME }\end{array}$ & 2.00 & $\begin{array}{l}125\left(1^{\text {st }} \text { cycle at } 0.1 \mathrm{C}\right) \text { vs. } \\
22\left(200^{\text {th }} \text { cycle at } 1 \mathrm{C}\right)\end{array}$ & 70 \\
\hline 88 & & 257 & $\begin{array}{l}\mathbf{8 8}(85) / \text { CC (10) / } \\
\text { PTFE (5) }\end{array}$ & & $\mathrm{Li}$ & $\begin{array}{l}1 \mathrm{M} \mathrm{LiPF}_{6} \text { in } \\
\mathrm{EC} / \mathrm{DMC} / \mathrm{DEC}\end{array}$ & 2.40 & $\begin{array}{l}118\left(1^{\text {st }} \text { cycle }\right) \text { vs. } 63\left(300^{\text {th }}\right. \\
\text { cycle }) \text { at } 100 \mathrm{~mA} \mathrm{~g}^{-1}\end{array}$ & 71 \\
\hline
\end{tabular}


Table S2 (continued)

\begin{tabular}{|c|c|c|c|c|c|c|c|c|c|}
\hline Number & Structure & $\begin{array}{l}\text { Specific capacity } \\
\text { b) } / \mathrm{mAh} \mathrm{g}^{-1}\end{array}$ & Cathode composition $^{\text {c) }}$ & $\begin{array}{l}\text { AM loading d)/ } \\
\mathrm{mg} \mathrm{cm}^{-2}\end{array}$ & Anode & Electrolyte & $\begin{array}{l}\text { Voltage }^{\mathrm{e})} / \mathrm{V} \\
\text { vs. } \mathrm{Li}^{-\mathrm{Li}^{+}}\end{array}$ & Cyclability / $\mathrm{mAh} \mathrm{g}^{-1}$ & Ref \\
\hline 89 & & & $\begin{array}{l}89(60) / A B(30) / \\
\text { PVDF (10) }\end{array}$ & & $\mathrm{Li}$ & $\begin{array}{l}1 \mathrm{MLiPF}_{6} \text { in } \\
\mathrm{EC} / \mathrm{DMC}\end{array}$ & 2.40 & $\begin{array}{l}130\left({ }^{\text {st }} \text { cycle }\right) \text { vs. } 109 \\
\left(300^{\text {th }} \text { cycle }\right) \text { at } 50 \mathrm{~mA} \mathrm{~g}^{-1}\end{array}$ & 72 \\
\hline 90 & & 292.1 & $\begin{array}{l}\mathbf{9 0}(80) / \mathrm{AB}(15) / \\
\text { PTFE }(5)\end{array}$ & & $\mathrm{Li}$ & $\begin{array}{l}1 \mathrm{M} \mathrm{LiPF}_{6} \text { in } \\
\mathrm{EC} / \mathrm{DMC}^{-} \mathrm{EMC}\end{array}$ & 2.30 & $\begin{array}{l}103\left({ }^{1 \text { st }} \text { cycle }\right) \text { vs. } 35\left(30^{\text {th }}\right. \\
\text { cycle) at } 0.2 \mathrm{C}\end{array}$ & 73 \\
\hline 91 & & 237 & 91 & & $\mathrm{Li}$ & $\begin{array}{l}1 \mathrm{M} \mathrm{LiPF}_{6} \text { in } \\
\mathrm{EC} / \mathrm{DMC}^{-}\end{array}$ & 2.50 & $\begin{array}{l}165\left(1^{\text {st }} \text { cycle }\right) \text { vs. } 101\left(10^{\text {th }}\right. \\
\text { cycle }) \text { at } 0.25 \mathrm{C}\end{array}$ & 74 \\
\hline 92 & & 207 & $\begin{array}{l}92(10) / \text { PVDF (10) / } \\
\text { MWCNT (80) }\end{array}$ & & $\mathrm{Li}$ & $\begin{array}{l}1 \mathrm{M} \mathrm{LiClO}_{4} \text { in } \\
\mathrm{EC} / \mathrm{DMC}\end{array}$ & 2.28 & $\begin{array}{l}130\left(1^{\text {st }} \text { cycle }\right) \text { vs. } 111 \\
\left(100^{\text {th }} \text { cycle }\right) \text { at } 5 \mathrm{C}\end{array}$ & 75 \\
\hline 93 & & 319 & $93(20) /$ CNT (27) & $0.31-1.34$ & $\mathrm{Li}$ & $\begin{array}{l}\text { 1.5 M LiTFSI in } \\
\mathrm{G}_{4} / \mathrm{EMC}\end{array}$ & 3.10 & $\begin{array}{l}396\left(1^{\text {st }} \text { cycle at } 0.2 \mathrm{C}\right) \mathrm{vs} . \\
320\left(3400^{\text {th }} \text { cycle at } 30 \mathrm{C}\right)\end{array}$ & 76 \\
\hline 94 & & 54.2 & $94(60) / C B(40)$ & & $\mathrm{Li}$ & $\begin{array}{l}1 \mathrm{M} \mathrm{LiClO}_{4} \text { in } \\
\mathrm{DOL} / \mathrm{DME}^{-}\end{array}$ & 2.45 & $\begin{array}{l}54.2\left(1^{\text {st }} \text { cycle at } 1 \mathrm{C}\right) \text { vs. } 52 \\
\left(3300^{\text {th }} \text { cycle at } 10 \mathrm{C}\right)\end{array}$ & 77 \\
\hline
\end{tabular}


Table S2 (continued)

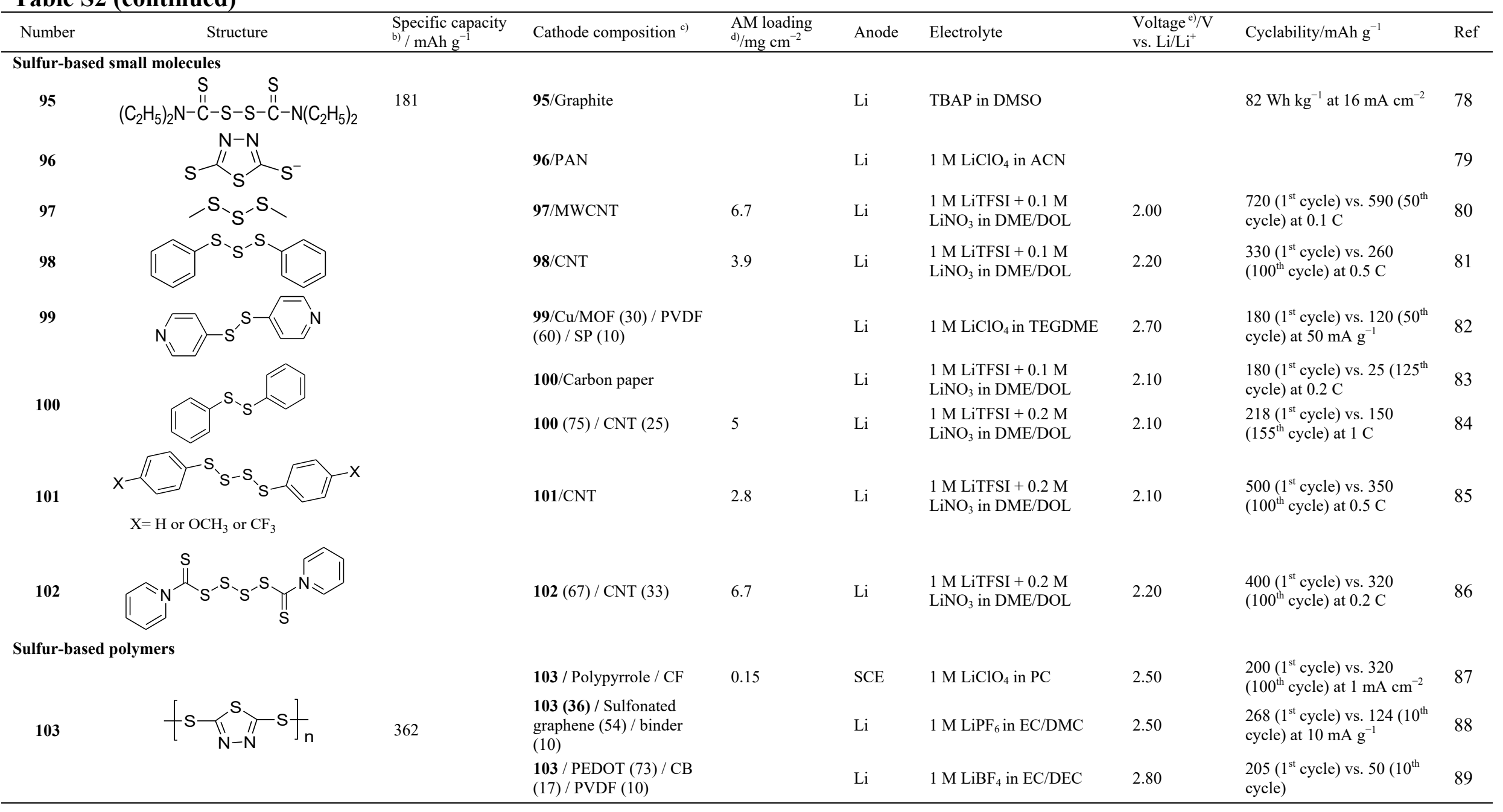


Table S2 (continued)

\begin{tabular}{|c|c|c|c|c|c|c|c|c|c|}
\hline Number & Structure & $\begin{array}{l}\text { Specific capacity } \\
\text { b) } / \mathrm{mAh} \mathrm{g}^{-1}\end{array}$ & Cathode composition ${ }^{\mathrm{c})}$ & $\begin{array}{l}\text { AM loading }{ }^{\mathrm{d} /} / \mathrm{mg} \\
\mathrm{cm}^{-2}\end{array}$ & Anode & Electrolyte & $\begin{array}{l}\text { Voltage }^{\mathrm{e})} / \mathrm{V} \\
\text { vs. } \mathrm{Li} / \mathrm{Li}^{+}\end{array}$ & Cyclability $/ \mathrm{mAh} \mathrm{g}^{-1}$ & Ref \\
\hline 104 & & 471 & $\begin{array}{l}\mathbf{1 0 4}(40) / \mathrm{AC}(40) / \\
\text { PTFE (20) }\end{array}$ & & $\mathrm{Li}$ & $\begin{array}{l}1 \mathrm{M} \mathrm{LiClO}_{4} \text { in } \\
\mathrm{EC} / \mathrm{DMC}^{-}\end{array}$ & 2.60 & $\begin{array}{l}422\left(2^{\text {nd }} \text { cycle }\right) \text { vs. } 170 \\
\left(44^{\text {th }} \text { cycle }\right) \text { at } 30 \mathrm{~mA} \mathrm{~g}^{-1}\end{array}$ & 90 \\
\hline 105 & & 261 & $\begin{array}{l}\mathbf{1 0 5}(42) \text { / C (48) / binder } \\
(10)\end{array}$ & & $\mathrm{Li}$ & $1 \mathrm{M} \mathrm{LiClO}_{4}$ in $\mathrm{EC} / \mathrm{PC}$ & 2.00 & $\begin{array}{l}230\left(1^{\text {st }} \text { cycle }\right) \text { vs. } 60\left(7^{\text {th }}\right. \\
\text { cycle }) \text { at } 0.1 \mathrm{~mA} \mathrm{~cm}^{-2}\end{array}$ & 91 \\
\hline 106 & & & $\begin{array}{l}\mathbf{1 0 6}(80) / \mathrm{CB}(10) / \\
\text { binder }(10)\end{array}$ & & $\mathrm{Ag} / \mathrm{AgCl}$ & $\begin{array}{l}1 \mathrm{M} \mathrm{LiPF}_{6} \text { in } \\
\mathrm{EC} / \mathrm{DMC}^{-}\end{array}$ & 2.70 & $\begin{array}{l}262\left(1^{\text {st }} \text { cycle }\right) \text { at } 10 \mathrm{~mA} \\
\mathrm{~g}^{-1}\end{array}$ & 92 \\
\hline & & & $\begin{array}{l}\mathbf{1 0 7}(40) / \mathrm{AB}(40) / \\
\text { PTFE (20) }\end{array}$ & & $\mathrm{Li}$ & $\begin{array}{l}1 \mathrm{M} \text { LiTFSI in } \\
\text { DME/DOL }\end{array}$ & 2.00 & $\begin{array}{l}290\left(1^{\text {st }} \text { cycle }\right) \text { vs. } 250\left(10^{\text {th }}\right. \\
\text { cycle }) \text { at } 20 \mathrm{~mA} \mathrm{~g}^{-1}\end{array}$ & 93 \\
\hline 107 & & 400 & $\begin{array}{l}\mathbf{1 0 7}(40) / \mathrm{AB}(40) / \\
\text { PTFE (20) }\end{array}$ & 2.7 & $\mathrm{Li}$ & $1 \mathrm{M}$ LiTFSI in DTST & 2.00 & $\begin{array}{l}318\left(1^{\text {st }} \text { cycle }\right) \text { at } 20 \mathrm{~mA} \\
\mathrm{~g}^{-1}\end{array}$ & 94 \\
\hline 108 & & 1067 & $\begin{array}{l}\mathbf{1 0 8}(70) / \mathrm{CB}(20) / \mathrm{LA}- \\
132(10)\end{array}$ & & $\mathrm{Li}$ & $\begin{array}{l}1 \mathrm{M} \mathrm{LiCF}_{3} \mathrm{SO}_{3} \text { in } \\
\mathrm{DOL} / \mathrm{DME}\end{array}$ & 2.2 & $\begin{array}{l}980\left(1^{\text {st }} \text { cycle }\right) \text { vs. } 412\left(20^{\text {th }}\right. \\
\text { cycle }) \text { at } 0.2 \mathrm{~mA} \mathrm{~cm}\end{array}$ & 95 \\
\hline 109 & or & & $\begin{array}{l}\mathbf{1 0 9}(65) \text { / Activated coal } \\
(30) \text { / PEO (5) }\end{array}$ & & $\mathrm{Li}$ & $\begin{array}{l}2 \mathrm{M} \text { LiTFSI in } \\
\mathrm{DME} / \mathrm{DOL}\end{array}$ & 1.8 & $\begin{array}{l}720\left(1^{\text {st }} \text { cycle }\right) \text { vs. } 212\left(30^{\text {th }}\right. \\
\text { cycle })\end{array}$ & 96 \\
\hline
\end{tabular}


Table S2 (continued)

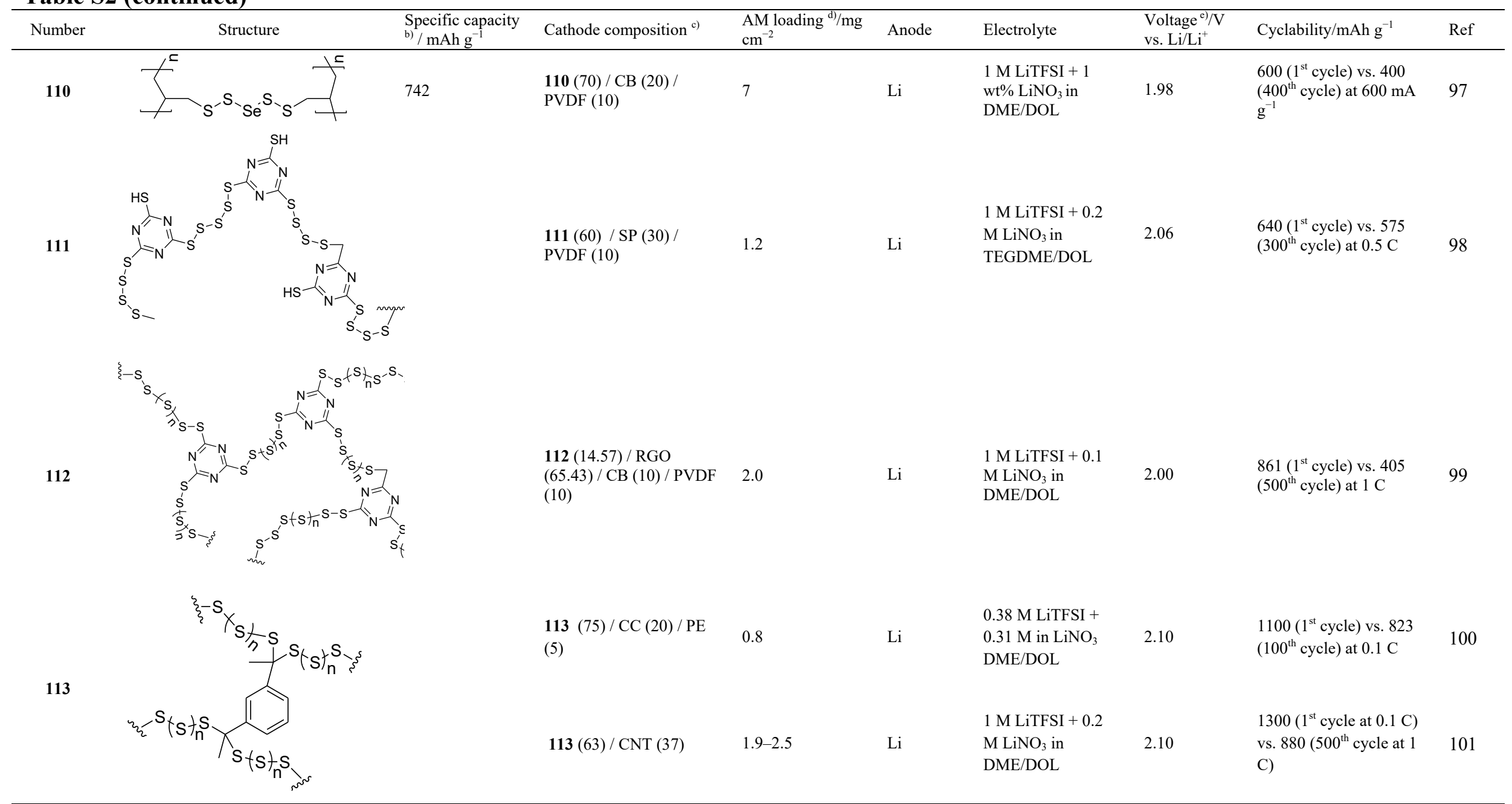


Table S2 (continued)

\begin{tabular}{|c|c|c|c|c|c|c|c|c|c|}
\hline Number & Structure & $\begin{array}{l}\text { Specific capacity } \\
\text { b) } / \mathrm{mAh} \mathrm{g}^{-1}\end{array}$ & Cathode composition ${ }^{\mathrm{c})}$ & $\begin{array}{l}\text { AM loading d)/mg } \\
\mathrm{cm}^{-2}\end{array}$ & Anode & Electrolyte & $\begin{array}{l}\text { Voltage }{ }^{\mathrm{e})} / \mathrm{V} \\
\text { vs. } \mathrm{Li}^{-} / \mathrm{Li}^{+}\end{array}$ & Cyclability/mAh g ${ }^{-1}$ & Ref \\
\hline 114 & & & $\begin{array}{l}114 \text { (63) / RGO (7) / } \\
\text { C65 (20) / PEO (10) }\end{array}$ & 1 & $\mathrm{Li}$ & $\begin{array}{l}0.25 \mathrm{M} \mathrm{LiTFSI}^{+} \\
0.1 \mathrm{M} \mathrm{LiNO}_{3} \text { in } \\
\mathrm{DME} / \mathrm{DOL}\end{array}$ & 2.10 & $\begin{array}{l}800\left(1^{\text {st }} \text { cycle }\right) \text { vs. } 700 \\
\left(100^{\text {th }} \text { cycle }\right) \text { at } 1000 \mathrm{~mA} \\
\mathrm{~g}^{-1}\end{array}$ & 102 \\
\hline 115 & & & $\begin{array}{l}115(70) / \text { CB (20) / } \\
\text { PVDF (10) }\end{array}$ & 1.6 & $\mathrm{Li}$ & $\begin{array}{l}1 \mathrm{M} \mathrm{LiTFSI}^{+} 0.1 \\
\mathrm{M} \mathrm{LiNO}_{3} \text { in } \\
\mathrm{DME} / \mathrm{DOL}\end{array}$ & 2.10 & $\begin{array}{l}800\left(1^{\text {st }} \text { cycle }\right) \text { vs. } 700 \\
\left(500^{\text {th }} \text { cycle }\right) \text { at } 1 \mathrm{C}\end{array}$ & 103 \\
\hline 116 & & & $\begin{array}{l}116(60) / C C(30) / \\
\text { PEO (10) }\end{array}$ & 2.0 & $\mathrm{Li}$ & $\begin{array}{l}1 \mathrm{M} \mathrm{LiTFSI}+2 \\
\mathrm{wt} \% \mathrm{LiNO}_{3} \text { in } \\
\mathrm{DME} / \mathrm{DOL}\end{array}$ & 2.10 & $\begin{array}{l}950\left(1^{\text {st }} \text { cycle }\right) \text { vs. } 700 \\
\left(500^{\text {th }} \text { cycle }\right) \text { at } 0.25 \mathrm{C}\end{array}$ & 104 \\
\hline 117 & & & $\begin{array}{l}\mathbf{1 1 7}(75) / \mathrm{CC}(20) / \mathrm{PE} \\
(5)\end{array}$ & 0.75 & $\mathrm{Li}$ & $\begin{array}{l}0.38 \mathrm{M} \mathrm{LiTFSI}^{+} \\
0.38 \mathrm{M} \mathrm{LiNO}_{3} \text { in } \\
\mathrm{DME} / \mathrm{DOL}\end{array}$ & 2.10 & $\begin{array}{l}1048\left(1^{\text {st }} \text { cycle }\right) \text { vs. } 485 \\
\left(1000^{\text {th }} \text { cycle }\right) \text { at } 0.2 \mathrm{C}\end{array}$ & 105 \\
\hline 118 & & & $\begin{array}{l}\mathbf{1 1 8}(40) / \text { CB (40) / } \\
\text { Teflon (20) }\end{array}$ & & $\mathrm{Li}$ & $\begin{array}{l}1 \mathrm{M} \text { LiTFSI in } \\
\text { DME/DOL }\end{array}$ & 2.20 & $\begin{array}{l}180\left(1^{\text {st }} \text { cycle }\right) \text { vs. } 100 \\
\left(20^{\text {th }} \text { cycle }\right) \text { at } 50 \mathrm{~mA} \mathrm{~g}^{-1}\end{array}$ & 106 \\
\hline
\end{tabular}


Table S2 (continued)

\begin{tabular}{|c|c|c|c|c|c|c|c|c|c|}
\hline Number & Structure & $\begin{array}{l}\text { Specific capacity }{ }^{b)} \\
/ \mathrm{mAh} \mathrm{g}^{-1}\end{array}$ & Cathode composition ${ }^{c)}$ & $\begin{array}{l}\text { AM loading } \mathrm{d} / \mathrm{mg} \\
\mathrm{cm}^{-2}\end{array}$ & Anode & Electrolyte & $\begin{array}{l}\text { Voltage } e^{\mathrm{e})} / \mathrm{V} \\
\text { vs. } \mathrm{Li}^{+\mathrm{Li}^{+}} \\
\end{array}$ & Cyclability $/ \mathrm{mAh} \mathrm{g}^{-1}$ & Ref \\
\hline 119 & & & $\begin{array}{l}\mathbf{1 1 9}(40) / \mathrm{CB}(40) / \\
\text { Teflon }(20)\end{array}$ & & $\mathrm{Li}$ & $\begin{array}{l}1 \mathrm{M} \text { LiTFSI in } \\
\text { DME/DOL }\end{array}$ & 2.20 & $\begin{array}{l}378\left(1^{\text {st }} \text { cycle }\right) \text { vs. } 300\left(20^{\text {th }}\right. \\
\text { cycle }) \text { at } 50 \mathrm{~mA} \mathrm{~g}^{-1}\end{array}$ & 106 \\
\hline 120 & & 570 & $\begin{array}{l}\mathbf{1 2 0}(40) / \text { CB }(40) / \\
\text { Teflon }(20)\end{array}$ & & $\mathrm{Li}$ & $\begin{array}{l}1 \mathrm{M} \text { LiTFSI in } \\
\text { DME/DOL }\end{array}$ & 2.00 & $\begin{array}{l}504\left(1^{\text {st }} \text { cycle }\right) \text { vs. } 100\left(50^{\text {th }}\right. \\
\text { cycle }) \text { at } 50 \mathrm{~mA} \mathrm{~g}^{-1}\end{array}$ & 107 \\
\hline 121 & & 505 & $\begin{array}{l}\mathbf{1 2 1}(40) / \text { CB }(40) / \\
\text { Teflon }(20)\end{array}$ & & $\mathrm{Li}$ & $\begin{array}{l}1 \mathrm{M} \text { LiTFSI in } \\
\text { DME/DOL }\end{array}$ & 2.00 & $\begin{array}{l}180\left(1^{\text {st }} \text { cycle }\right) \text { vs. } 100\left(50^{\text {th }}\right. \\
\text { cycle }) \text { at } 50 \mathrm{~mA} \mathrm{~g}^{-1}\end{array}$ & 107 \\
\hline 122 & & & $\begin{array}{l}\mathbf{1 2 2}(40) / \text { CB }(40) / \\
\text { Teflon }(20)\end{array}$ & & $\mathrm{Li}$ & $\begin{array}{l}1 \mathrm{M} \text { LiTFSI in } \\
\text { DME/DOL }\end{array}$ & 2.25 & $\begin{array}{l}632\left(2^{\text {nd }} \text { cycle }\right) \text { vs. } 275\left(40^{\text {th }}\right. \\
\text { cycle }) \text { at } 50 \mathrm{~mA} \mathrm{~g}^{-1}\end{array}$ & 108 \\
\hline 123 & & 1116 & $\begin{array}{l}\mathbf{1 2 3}(40) / \text { CB }(40) / \\
\text { Teflon }(20)\end{array}$ & & $\mathrm{Li}$ & $\begin{array}{l}1 \mathrm{M} \text { LiTFSI in } \\
\text { DME/DOL }\end{array}$ & 2.30 & $\begin{array}{l}820\left(1^{\text {st }} \text { cycle }\right) \text { vs. } 560\left(20^{\text {th }}\right. \\
\text { cycle }) \text { at } 50 \mathrm{~mA} \mathrm{~g}^{-1}\end{array}$ & 109 \\
\hline 124 & & & $\begin{array}{l}\mathbf{1 2 4}(40) / \text { CB }(40) / \\
\text { Teflon }(20)\end{array}$ & & $\mathrm{Li}$ & $\begin{array}{l}1 \mathrm{M} \text { LiTFSI in } \\
\text { DME/DOL }\end{array}$ & 2.30 & $\begin{array}{l}200\left(1^{\text {st }} \text { cycle) vs. } 90\left(20^{\text {th }}\right.\right. \\
\text { cycle }) \text { at } 50 \mathrm{~mA} \mathrm{~g}^{-1}\end{array}$ & 110 \\
\hline \multicolumn{10}{|c|}{ Radical polymers } \\
\hline \multirow{4}{*}{125} & & \multirow{4}{*}{111} & $\begin{array}{l}\mathbf{1 2 5}(50) / \text { VGCF (45)/ } \\
\text { PTFE (1) / CMC (4) }\end{array}$ & & $\mathrm{Li}$ & $\begin{array}{l}1 \mathrm{M} \mathrm{LiPF}_{6} \text { in } \\
\mathrm{EC} / \mathrm{DEC}\end{array}$ & 3.51 & $\begin{array}{l}110\left(1^{\text {st }} \text { cycle }\right) \text { vs. } 99\left(100^{\text {th }}\right. \\
\text { cycle }) \text { at } 1 \mathrm{C}\end{array}$ & 111 \\
\hline & & & $\begin{array}{l}\mathbf{1 2 5}(50) / \text { VGCF (45)/ } \\
\text { PTFE (1) / CMC (4) }\end{array}$ & & Graphite & $\begin{array}{l}1 \mathrm{M} \mathrm{LiPF}_{6} \text { in } \\
\mathrm{EC} / \mathrm{DEC}\end{array}$ & 3.51 & $\begin{array}{l}108\left(1^{\text {st }} \text { cycle }\right) \text { vs. } 86\left(100^{\text {th }}\right. \\
\text { cycle }) \text { at } 80 \mathrm{~mA} \mathrm{~g}^{-1}\end{array}$ & 112 \\
\hline & & & $\begin{array}{l}\mathbf{1 2 5}(50) / \text { CB (30)/ } \\
\text { PVDF-HFP (20) }\end{array}$ & 3.15 & $\mathrm{Li}$ & $\begin{array}{l}1 \mathrm{M} \mathrm{LiPF}_{6} \text { in } \\
\mathrm{EC} / \mathrm{DEC}\end{array}$ & 3.60 & $\begin{array}{l}109\left(1^{\text {st }} \text { cycle }\right) \text { vs. } 66\left(150^{\text {th }}\right. \\
\text { cycle }) \text { at } 50 \mathrm{C}\end{array}$ & 113 \\
\hline & $0_{\bullet}$ & & $\begin{array}{l}\mathbf{1 2 5}(60) / \mathrm{CB}(20) / \\
\text { PVDF-HFP (10) }\end{array}$ & 9.0 & $\mathrm{Li}$ & $\begin{array}{l}1 \mathrm{M} \mathrm{LiPF}_{6} \text { in } \\
\mathrm{EC} / \mathrm{DMC}^{2}\end{array}$ & 3.60 & $\begin{array}{l}111\left(1^{\text {st }} \text { cycle }\right) \text { vs. } 110 \\
\left(100^{\text {th }} \text { cycle }\right) \text { at } 1 \mathrm{C}\end{array}$ & 114 \\
\hline
\end{tabular}


Table S2 (continued)

\begin{tabular}{|c|c|c|c|c|c|c|c|c|c|}
\hline Number & Structure & $\begin{array}{l}\text { Specific capacity } \\
\text { b) } / \mathrm{mAh} \mathrm{g}^{-1}\end{array}$ & Cathode composition ${ }^{c)}$ & $\begin{array}{l}\text { AM loading d)/mg } \\
\mathrm{cm}^{-2}\end{array}$ & Anode & Electrolyte & $\begin{array}{l}\text { Voltage } e^{\mathrm{e})} / \mathrm{V} \\
\text { vs. } \mathrm{Li}^{-} \mathrm{Li}^{+}\end{array}$ & Cyclability/mAh g ${ }^{-1}$ & Ref \\
\hline \multirow{6}{*}{125} & & \multirow{6}{*}{ f } & $\begin{array}{l}\mathbf{1 2 5}(30) / \mathrm{BP}-2000(60) \\
\text { / PVDF (10) }\end{array}$ & & $\mathrm{Li}$ & $\begin{array}{l}1 \mathrm{M} \mathrm{LiPF}_{6} \text { in } \\
\mathrm{EC} / \mathrm{DMC}^{-}\end{array}$ & 3.60 & $\begin{array}{l}133\left(1^{\text {st }} \text { cycle }\right) \text { vs. } 106 \\
\left(500^{\text {th }} \text { cycle }\right) \text { at } 1 \mathrm{C}\end{array}$ & 115 \\
\hline & & & $\begin{array}{l}125 \text { (27) / Graphite (46) } \\
\text { / CB (8) / PVDF (19) }\end{array}$ & & $\mathrm{Li}$ & $\begin{array}{l}1 \mathrm{M} \mathrm{LiPF}_{6} \text { in } \\
\mathrm{EC} / \mathrm{DMC}^{-}\end{array}$ & 3.60 & $\begin{array}{l}105\left(1^{\text {st }} \text { cycle }\right) \text { vs. } 100 \\
\left(200^{\text {th }} \text { cycle }\right) \text { at } 1 \mathrm{C}\end{array}$ & 116 \\
\hline & & & $125(70) / \operatorname{VGCF}(30)$ & 4.69 & $\mathrm{Li}$ & $\begin{array}{l}1.5 \mathrm{M} \text { LiFSI in } \\
\mathrm{EC} / \mathrm{DMC}\end{array}$ & 3.50 & $105\left(1^{\text {st }}\right.$ cycle $)$ at $1 \mathrm{C}$ & 117 \\
\hline & & & $\begin{array}{l}\mathbf{1 2 5}(10) / \text { SP (80) / } \\
\text { PVDF (10) }\end{array}$ & 0.24 & $\mathrm{Li}$ & $\begin{array}{l}1 \text { M LiTFSI in } \\
\text { BMPTFSI }\end{array}$ & 3.60 & $\begin{array}{l}90\left(1^{\text {st }} \text { cycle }\right) \text { vs. } 100 \\
\left(200^{\text {th }} \text { cycle }\right) \text { at } 10 \mathrm{C}\end{array}$ & 118 \\
\hline & & & 125 & & & & & & 119 \\
\hline & & & $\begin{array}{l}\mathbf{1 2 5}(60) / \mathrm{SP}(35) / \\
\operatorname{PVDF}(5)\end{array}$ & 0.4 & $\mathrm{Li}$ & $\mathrm{Pyr}_{14}$ TFSI in PC & 3.76 & $\begin{array}{l}106\left(1000^{\text {th }} \text { cycle }\right) \text { vs. } 78 \\
\left(10000^{\text {th }} \text { cycle }\right) \text { at } 10 \mathrm{C}\end{array}$ & 120 \\
\hline 126 & & & $\mathbf{1 2 6}(50)$ / PTMA (50) & 0.5 & $\mathrm{Ag} / \mathrm{AgCl}$ & $1 \mathrm{M} \mathrm{LiClO}_{4}$ in $\mathrm{H}_{2} \mathrm{O}$ & 3.51 & $\begin{array}{l}80\left(1^{\text {st }} \text { cycle }\right) \text { vs. } 46 \\
\left(1000^{\text {th }} \text { cycle }\right) \text { at } 50 \mathrm{mV} \\
\mathrm{s}^{-1}\end{array}$ & 121 \\
\hline 127 & & 109 & 127 / Carbon & & $\mathrm{Ag} / \mathrm{AgCl}$ & $\begin{array}{l}1 \mathrm{M} \mathrm{LiPF}_{6} \text { in } \\
\mathrm{EC} / \mathrm{DEC}\end{array}$ & 3.60 & $106\left(1^{\text {st }}\right.$ cycle $)$ at $10 \mathrm{C}$ & 122 \\
\hline 128 & & 135 & 128 & & $\mathrm{Ag} / \mathrm{AgCl}$ & $0.1 \mathrm{M} \mathrm{NaCl}$ in $\mathrm{H}_{2} \mathrm{O}$ & 3.96 & $\begin{array}{l}131\left(1^{\text {st }} \text { cycle }\right) \text { vs. } 98 \\
\left(1000^{\text {th }} \text { cycle }\right) \text { at } 10 \mathrm{~mA} \\
\mathrm{~cm}^{-2}\end{array}$ & 123 \\
\hline 129 & & 100 & $\mathbf{1 2 9}(90)$ / SWCNT (10) & & $\mathrm{Li}$ & $\begin{array}{l}1 \mathrm{M} \mathrm{LiFP}_{6} \text { in } \\
\mathrm{EC} / \mathrm{DEC}\end{array}$ & 3.50 & $\begin{array}{l}80\left(1^{\text {st }} \text { cycle }\right) \text { vs. } 56 \\
\left(200^{\text {th }} \text { cycle }\right) \text { at } 1 \mathrm{C}\end{array}$ & 124 \\
\hline
\end{tabular}


Table S2 (continued)

\begin{tabular}{|c|c|c|c|c|c|c|c|c|c|}
\hline Number & Structure & $\begin{array}{l}\text { Specific capacity } \\
\text { b) } / \mathrm{mAh} \mathrm{g}^{-1}\end{array}$ & Cathode composition ${ }^{\mathrm{c})}$ & $\begin{array}{l}\text { AM loading }{ }^{\mathrm{d}} / \mathrm{mg} \\
\mathrm{cm}^{-2}\end{array}$ & Anode & Electrolyte & $\begin{array}{l}\text { Voltage }{ }^{\mathrm{e})} / \mathrm{V} \\
\text { vs. } \mathrm{Li}^{-\mathrm{Li}^{+}}\end{array}$ & Cyclability $/ \mathrm{mAh} \mathrm{g}^{-1}$ & Ref \\
\hline 130 & & 76 & 130 & & $\mathrm{Li}$ & $\begin{array}{l}1 \mathrm{M} \mathrm{LiPF}_{6} \text { in } \\
\mathrm{EC} / \mathrm{DEC}\end{array}$ & 3.60 & $\begin{array}{l}47\left(1^{\text {st }} \text { cycle }\right) \text { vs. } 38\left(50^{\text {th }}\right. \\
\text { cycle }) \text { at } 0.1 \mathrm{C}\end{array}$ & 125 \\
\hline 131 & & 45 & 131 (50) / MWNT (50) & 1 & $\mathrm{Li}$ & $\begin{array}{l}1 \mathrm{M} \text { LiTFSI in } \\
\mathrm{EC} / \mathrm{DEC} / \mathrm{DMC}\end{array}$ & 3.62 & $\begin{array}{l}60\left(1^{\text {st }} \text { cycle }\right) \text { vs. } 60 \\
\left(1300^{\text {th }} \text { cycle }\right) \text { at } 60 \mathrm{C}\end{array}$ & 126 \\
\hline \multicolumn{10}{|c|}{ Other organic compounds } \\
\hline 132 & & 255 & $\begin{array}{l}\mathbf{1 3 2}(60) \text { / SP (30) / } \\
\text { PVDF (10) }\end{array}$ & 1.2 & $\mathrm{Li}$ & $\begin{array}{l}1 \mathrm{M} \text { LiTFSI } \\
\text { in DOL/DME }\end{array}$ & 1.68 & $\begin{array}{l}256\left(1^{\text {st }} \text { cycle }\right) \text { vs. } 220 \\
\left(100^{\text {th }} \text { cycle }\right) \text { at } 100 \mathrm{~mA} \\
\mathrm{~g}^{-1}\end{array}$ & 127 \\
\hline 133 & & & $\begin{array}{l}\mathbf{1 3 3}(48.1) / \mathrm{KB}(47.9) / \\
\text { PTFE (4) }\end{array}$ & & $\mathrm{Li}$ & $\begin{array}{l}1 \mathrm{M} \mathrm{LiClO}_{4} \\
\text { in EC/DEC }\end{array}$ & 2.41 & $\begin{array}{l}300\left({ }^{\text {st }} \text { cycle }\right) \text { vs. } 220 \\
\left(30^{\text {th }} \text { cycle }\right) \text { at } 0.2 \mathrm{C}\end{array}$ & 128 \\
\hline 134 & & 139 & $\begin{array}{l}\mathbf{1 3 4}(50) \text { / KB (20) / } \\
\text { VGCF (20) / PVDF (10) }\end{array}$ & & $\mathrm{Li}$ & $\begin{array}{l}1 \mathrm{M} \mathrm{LiPF}_{6} \text { in } \\
\mathrm{EC} / \mathrm{DEC}\end{array}$ & 3.70 & $\begin{array}{l}125\left(4^{\text {th }} \text { cycle }\right) \text { vs. } 117 \\
\left(50^{\text {th }} \text { cycle }\right) \text { at } 20 \mathrm{~mA} \mathrm{~g}^{-1}\end{array}$ & 129 \\
\hline 135 & & 132 & $\begin{array}{l}\mathbf{1 3 5}(10) / \text { VGCF (80) / } \\
\text { PVDF (10) }\end{array}$ & & $\mathrm{Li}$ & $\begin{array}{l}1 \mathrm{M} \mathrm{LiClO}_{4} \text { in } \\
\mathrm{PC} / \mathrm{DME}\end{array}$ & 3.10 & $\begin{array}{l}108\left(1^{\text {st }} \text { cycle }\right) \text { vs. } 82 \\
\left(250^{\text {th }} \text { cycle }\right) \text { at } 1 \mathrm{C}\end{array}$ & 130 \\
\hline
\end{tabular}


Table S2 (continued)

\begin{tabular}{|c|c|c|c|c|c|c|c|c|c|}
\hline Number & Structure & $\begin{array}{l}\text { Specific capacity } \\
\text { b) } / \mathrm{mAh} \mathrm{g}^{-1}\end{array}$ & Cathode composition $^{\text {c) }}$ & $\begin{array}{l}\text { AM loading } \mathrm{d} / \mathrm{mg} \\
\mathrm{cm}^{-2}\end{array}$ & Anode & Electrolyte & $\begin{array}{l}\text { Voltage }^{\mathrm{e})} / \mathrm{V} \\
\text { vs. } \mathrm{Li} / \mathrm{Li}^{+}\end{array}$ & Cyclability $/ \mathrm{mAh} \mathrm{g}^{-1}$ & Ref \\
\hline 136 & & 160 & $\begin{array}{l}\mathbf{1 3 6} \text { / VGCF (30) / SP } \\
\text { (30) / PVDF (10) }\end{array}$ & & $\mathrm{Li}$ & $0.1 \mathrm{M} \mathrm{LiClO}_{4}$ in $\mathrm{PC}$ & 2.60 & $\begin{array}{l}156\left(1^{\text {st }} \text { cycle }\right) \text { vs. } 141 \\
\left(500^{\text {th }} \text { cycle }\right) \text { at } 1 \mathrm{C}\end{array}$ & 131 \\
\hline 137 & & 108 & $\begin{array}{l}\mathbf{1 3 7}(70) / \mathrm{AB}(20) / \\
\text { PTFE (10) }\end{array}$ & & $\mathrm{Li}$ & $\begin{array}{l}1 \mathrm{M} \mathrm{LiPF}_{6} \text { in } \\
\mathrm{EC} / \mathrm{DMC}\end{array}$ & 3.80 & $\begin{array}{l}91\left(2^{\text {nd }} \text { cycle }\right) \text { vs. } 84 \\
\left(1000^{\text {th }} \text { cycle }\right) \text { at } 20 \mathrm{C}\end{array}$ & 132 \\
\hline 138 & & 175 & 138 & & $\mathrm{Li}$ & $0.1 \mathrm{M} \mathrm{NaCl}$ in $\mathrm{H}_{2} \mathrm{O}$ & 2.66 & $\begin{array}{l}165\left(2^{\text {nd }} \text { cycle }\right) \text { vs. } 149 \\
\left(2000^{\text {th }} \text { cycle }\right) \text { at } 60 \mathrm{C}\end{array}$ & 133 \\
\hline 139 & & & $\begin{array}{l}\mathbf{1 3 9}(50) / \mathrm{AB}(40) / \\
\mathrm{PVDF}(10)\end{array}$ & & $\mathrm{Li}$ & $\begin{array}{l}1 \mathrm{M} \mathrm{LiPF}_{6} \text { in } \\
\mathrm{EC} / \mathrm{DMC}^{-}\end{array}$ & 3.60 & $\begin{array}{l}129\left(1^{\text {st }} \text { cycle }\right) \text { vs. } 118 \\
\left(50^{\text {th }} \text { cycle }\right) \text { at } 20 \mathrm{~mA} \mathrm{~g}^{-1}\end{array}$ & 134 \\
\hline 140 & & 73 & $\begin{array}{l}\mathbf{1 4 0}(50) / \mathrm{SP}(47) / \\
\operatorname{PVDF}(3)\end{array}$ & & $\mathrm{Li}$ & $\begin{array}{l}1 \mathrm{M} \mathrm{LiPF}_{6} \text { in } \\
\mathrm{EC} / \mathrm{DMC}\end{array}$ & 4.05 & $\begin{array}{l}66\left(14^{\text {th }} \text { cycle }\right) \text { vs. } 20 \\
\left(100^{\text {th }} \text { cycle }\right) \text { at } 1 \mathrm{C}\end{array}$ & 135 \\
\hline 141 & & 112 & $\begin{array}{l}141(50) / \text { CB (47) / } \\
\text { PVDF (3) }\end{array}$ & $0.07-0.37$ & $\mathrm{Li}$ & $\begin{array}{l}1 \mathrm{M} \mathrm{LiPF}_{6} \text { in } \\
\mathrm{EC} / \mathrm{DMC}^{-}\end{array}$ & 3.56 & $\begin{array}{l}50\left(1^{\text {st }} \text { cycle }\right) \text { vs. } 56 \\
\left(1000^{\text {th }} \text { cycle }\right) \text { at } 1 \mathrm{C}\end{array}$ & 136 \\
\hline
\end{tabular}




\section{Table S2 (continued)}

\begin{tabular}{|c|c|c|c|c|c|c|c|c|c|}
\hline Number & Structure & $\begin{array}{l}\text { Specific capacity } \\
\text { b) } / \mathrm{mAh} \mathrm{g}^{-1}\end{array}$ & Cathode composition ${ }^{\mathrm{c}}$ & $\begin{array}{l}\text { AM loading }{ }^{\mathrm{d})} / \mathrm{mg} \\
\mathrm{cm}^{-2}\end{array}$ & Anode & Electrolyte & $\begin{array}{l}\text { Voltage }^{\mathrm{e})} / \mathrm{V} \\
\text { vs. } \mathrm{Li}^{+} / \mathrm{Li}^{+}\end{array}$ & Cyclability/mAh g ${ }^{-1}$ & $\operatorname{Ref}$ \\
\hline 142 & & 126 & $\begin{array}{l}\mathbf{1 4 2}(10) / \mathrm{CF}(80) / \mathrm{FP} \\
(10)\end{array}$ & & $\mathrm{Li}$ & $\begin{array}{l}1.5 \mathrm{M} \mathrm{LiPF}_{6} \mathrm{EC} / \mathrm{D} \\
\mathrm{EC}\end{array}$ & 3.20 & $\begin{array}{l}105\left(1^{\text {st }} \text { cycle }\right) \text { vs. } 100 \\
\left(300^{\mathrm{th}} \text { cycle }\right) \text { at } 200 \mathrm{~mA} \\
\mathrm{~g}^{-1}\end{array}$ & 137 \\
\hline 143 & & 73.9 & $\begin{array}{l}\mathbf{1 4 3}(28) \text { / Soot (8) / } \\
\text { Graphite (48) / PVDF } \\
\text { (16) }\end{array}$ & 1.43 & $\mathrm{Li}$ & $\begin{array}{l}1 \mathrm{M} \mathrm{LiPF}_{6} \text { in } \\
\mathrm{EC} / \mathrm{DMC}\end{array}$ & 3.85 & $\begin{array}{l}52\left(1^{\text {st }} \text { cycle }\right) \text { vs. } 46\left(90^{\text {th }}\right. \\
\text { cycle }) \text { at } 1 \mathrm{C}\end{array}$ & 138 \\
\hline 144 & & 1231 & $\begin{array}{l}144(70) / \mathrm{KB}(20) / \\
\mathrm{CMC}(10)\end{array}$ & $1.4-1.9$ & $\mathrm{Li}$ & $\begin{array}{l}1 \mathrm{M} \mathrm{LiPF}_{6} \text { in } \\
\mathrm{EC} / \mathrm{DEC}\end{array}$ & 1.00 & $\begin{array}{l}1050\left(1^{\text {st }} \text { cycle }\right) \text { vs. } 486 \\
\left(1000^{\text {th }} \text { cycle }\right) \text { at } 1000 \\
\mathrm{~mA} \mathrm{~g} \mathrm{~g}^{-1}\end{array}$ & 139 \\
\hline
\end{tabular}

a) The abbreviations are listed as below: AB (acetylene black), AC (acetylene carbon), ACN (acetonitrile), AG (activated graphene), AHE (autogenous hydrogen electrode), BMPTFSI ( $N$-butyl- $N$-methylpyrrolidinium bis(trifluoromethylsulfonyl)imide), BP-2000 (black pearl 2000 carbon black), CB (carbon black), CC (conductive carbon), CF (carbon fiber), CMC (sodium carboxymethyl cellulose), CMK-3 (ordered mesoporous carbon), CNT (carbon nanotube), DEC (diethyl carbonate), DIOX (diethylene dioxide), DMC (dimethyl carbonate), DME (dimethoxyethane), DMSO (dimethyl sulfoxide), DOL (dioxolane), DTST (12,12-diethyl-2,5,8trioxa-12-silatetradecane), EC (ethylene carbonate), FGS (functionalized graphene sheets), FP (fluorinated polyolefin), FWCNT (few-walled carbon nanotubes), KB (Ketjen black), LiFSI [lithium bis(fluorosulfonyl)imide], LiTFSI [lithium bis(trifluoromethylsulfonil)imide], $\mathrm{LiPF}_{6}$ (lithium hexafluorophosphate), $\mathrm{LiNO}_{3}$ (lithium nitrate), $\mathrm{LiClO}_{4}($ lithium perchlorate), $\mathrm{LiBF} 4$ (lithium tetrafluoroborate), Me-THF (2methyltetrahydrofuran), MWCNT (multiwalled carbon nanotubes), PAN (polyaniline), PC (propylene carbonate), PE (polyethylene), PEDOT [poly(3,4-ethylenedioxythiophene)], PEO [poly(ethylene oxide)], PTFE [poly(tetrafluorethylene)], PTMA (poly-TEMPO-methacrylate), Pyr14TFSI [1-butyl-1-methylpyrrolidinium bis(trifluoromethylsulfonyl)imide], RGO (reduced graphene oxide), PVDF [poly(vinylidene fluoride)], PVDF-HFP [poly(vinylidene fluoride-co-hexafluoropropylene)], SA (sodium alginate), SCE (standard hydrogen electrode), SWCNT (single-wall carbon nanotube), TBAClO 4 (tetrabutylammonium perchlorate), TBAP (tetrabutylammonium perchlorate), TEGDME (triethylene glycol dimethyl ether), TG (tetraglyme), VGCF (vapor grown carbon fibers).

b) Theoretic specific capacity of the presented organic compound.

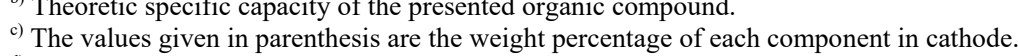

c) The values given in parenthesis are the weight p
d) The mass loading of active materials in cathode.

e) Average working voltage of the presented organic compound. 
Table S3 Properties of organic materials developed for Na-based and K-based batteries. ${ }^{\text {a) }}$

\begin{tabular}{|c|c|c|c|c|c|c|c|c|c|}
\hline Number & Structure & $\begin{array}{l}\text { Specific capacity } \\
\text { b) } / \mathrm{mAh} \mathrm{g}^{-1}\end{array}$ & $\begin{array}{l}\text { Cathode } \\
\text { composition }^{c}\end{array}$ & $\begin{array}{l}\text { AM loading } \\
\text { d) } / \mathrm{mg} \mathrm{cm}^{-2}\end{array}$ & Anode & Electrolyte & $\begin{array}{l}\text { Voltage }{ }^{\mathrm{e})} / \mathrm{V} \\
\text { vs. } \mathrm{Na} / \mathrm{Na}^{+}\end{array}$ & Cyclability/mAh g ${ }^{-1}$ & Ref \\
\hline \multicolumn{10}{|c|}{ Sodium-based organic battery } \\
\hline 145 & & 187 & $\begin{array}{l}145 \text { (65) / SP (30) / } \\
\text { PVDF (5) }\end{array}$ & $1-2$ & $\mathrm{Na}$ & $\begin{array}{l}1 \mathrm{M} \mathrm{NaClO}_{4} \text { in } \\
\mathrm{EC} / \mathrm{DMC}\end{array}$ & 2.30 & $\begin{array}{l}183\left(1^{\text {st }} \text { cycle }\right) \text { vs. } 154 \\
\left(100^{\text {th }} \text { cycle }\right) \text { at } 0.1 \mathrm{C}\end{array}$ & 140 \\
\hline 146 & & 250 & $\begin{array}{l}146(70) / C B ~ / ~(20) \\
\text { / PVDF (10) }\end{array}$ & 0.8 & $\mathrm{Na}$ & $\begin{array}{l}1 \mathrm{M} \mathrm{NaClO}_{4} \text { in } \\
\mathrm{EC} / \mathrm{PC}+5 \% \mathrm{FEC}\end{array}$ & 2.10 & $\begin{array}{l}190\left(\left(^{\text {st }} \text { cycle }\right) \text { vs. } 95\right. \\
\left(100^{\text {th }} \text { cycle }\right) \text { at } 0.1 \mathrm{C}\end{array}$ & 141 \\
\hline 147 & & 214 & $\begin{array}{l}147(42) \text { / CMK-3 } \\
\text { (56) }\end{array}$ & & $\mathrm{Na}$ & $\begin{array}{l}1 \mathrm{M} \mathrm{NaClO}_{4} \\
\text { in } \mathrm{EC} / \mathrm{PC} / \mathrm{DMC}\end{array}$ & 2.72 & $\begin{array}{l}160\left(\left(^{\text {st }} \text { cycle }\right) \text { vs. } 40\right. \\
\left(20^{\text {th }} \text { cycle }\right) \text { at } 19 \mathrm{~mA} \\
\mathrm{~g}^{-1}\end{array}$ & 142 \\
\hline 148 & & 137 & $\begin{array}{l}148 \text { (70) / SP (20) / } \\
\text { PVDF (10) }\end{array}$ & 2 & $\mathrm{Na}$ & $\begin{array}{l}1 \mathrm{M} \mathrm{NaClO}_{4} \text { in } \\
\mathrm{EC} / \mathrm{DEC}\end{array}$ & 2.30 & $\begin{array}{l}145\left(\left(^{\text {st }} \text { cycle at } 10 \mathrm{~mA}\right.\right. \\
\left.\mathrm{g}^{-1}\right) \text { vs. } 100\left(200^{\text {th }} \text { cycle }\right. \\
\text { at } 1.4 \mathrm{C})\end{array}$ & 143 \\
\hline 149 & & 80 & $\begin{array}{l}149(60) / \mathrm{AB}(30) / \\
\mathrm{PVDF}(10)\end{array}$ & 1.4 & $\mathrm{Na}$ & $\begin{array}{l}1 \mathrm{M} \mathrm{NaPF}_{6} \\
\text { in PC }\end{array}$ & 2.09 & $90\left(1^{\text {st }}\right.$ cycle $)$ at $0.25 \mathrm{C}$ & 144 \\
\hline 150 & & 137 & $\begin{array}{l}\mathbf{1 5 0}(70) / \text { SP (20) / } \\
\text { PTFE (10) }\end{array}$ & & $\mathrm{Na}$ & $\begin{array}{l}1 \mathrm{M} \mathrm{NaClO}_{4} \\
\text { in EC/PC/DEC }\end{array}$ & 1.70 & $\begin{array}{l}138.6\left(1^{\text {st }} \text { cycle at } 10\right. \\
\left.\mathrm{mA} \mathrm{g}^{-1}\right) \text { vs. } 124.7 \\
\left(300^{\text {th }} \text { cycle at } 200 \mathrm{~mA}\right. \\
\left.\mathrm{g}^{-1}\right)\end{array}$ & 145 \\
\hline 151 & & 250 & 151 (41) / CNT (59) & & $\mathrm{Na}$ & $\begin{array}{l}1 \mathrm{M} \mathrm{NaPF}_{6} \text { in } \\
\text { TEGDME }\end{array}$ & 1.90 & $\begin{array}{l}255\left(1^{\text {st }} \text { cycle }\right) \text { at } 50 \\
\mathrm{~mA} \mathrm{~g}^{-1}\end{array}$ & 146 \\
\hline 152 & & 248.6 & $\begin{array}{l}152(60) / A B(30) / \\
\text { PVDF (10) }\end{array}$ & & $\mathrm{Na}$ & $\begin{array}{l}1 \mathrm{M} \mathrm{NaClO}_{4} \text { in } \\
\mathrm{EC} / \mathrm{DMC}\end{array}$ & 1.94 & $\begin{array}{l}107.7\left(1^{\text {st }} \text { cycle at } 25\right. \\
\left.\mathrm{mA} \mathrm{g}^{-1}\right) \text { vs. } 89.4\left(150^{\text {th }}\right. \\
\left.\text { cycle at } 200 \mathrm{~mA} \mathrm{~g}^{-1}\right)\end{array}$ & 147 \\
\hline 153 & & 260 & $\begin{array}{l}153(60) / A B(30) / \\
\text { PVDF (10) }\end{array}$ & 1.4 & $\mathrm{Na}$ & $1 \mathrm{M} \mathrm{NaPF}_{6}$ in $\mathrm{PC}$ & 2.15 & $\begin{array}{l}126\left(1^{\text {st }} \text { cycle }\right) \text { vs. } 113 \\
\left(50^{\text {th }} \text { cycle }\right) \text { at } 100 \mathrm{~mA} \\
\mathrm{~g}^{-1}\end{array}$ & 148 \\
\hline
\end{tabular}




\section{Table S3 (continued)}

\begin{tabular}{|c|c|c|c|c|c|c|c|c|c|}
\hline Number & Structure & $\begin{array}{l}\text { Specific capacity } \\
\text { b) } / \mathrm{mAh} \mathrm{g}^{-1}\end{array}$ & $\begin{array}{l}\text { Cathode composition } \\
\text { c) }\end{array}$ & $\begin{array}{l}\text { AM loading } \\
\text { d) } / \mathrm{mg} \mathrm{cm}^{-2}\end{array}$ & Anode & Electrolyte & $\begin{array}{l}\text { Voltage }^{\mathrm{e})} / \mathrm{V} \\
\text { vs. } \mathrm{Na} / \mathrm{Na}^{+}\end{array}$ & Cyclability $/ \mathrm{mAh} \mathrm{g}^{-1}$ & Ref \\
\hline 154 & & & $\begin{array}{l}\mathbf{1 5 4}(60) / \mathrm{AB}(30) / \\
\mathrm{PVDF}(10)\end{array}$ & & $\mathrm{Na}$ & $\begin{array}{l}1 \mathrm{M} \mathrm{NaPF}_{6} \\
\text { in } \mathrm{EC} / \mathrm{DMC}\end{array}$ & 1.89 & $\begin{array}{l}133\left(1^{\text {st }} \text { cycle }\right) \text { vs. } 90 \\
\left(105^{\text {th }} \text { cycle }\right) \text { at } 200 \mathrm{~mA} \\
\mathrm{~g}^{-1}\end{array}$ & 149 \\
\hline 155 & & & $\begin{array}{l}155(40) / \text { CB (40) / } \\
\text { PVDF (20) }\end{array}$ & & $\mathrm{Na}$ & $\begin{array}{l}0.5 \mathrm{M} \mathrm{NaPF}_{6} \\
\text { in DOL/DME }\end{array}$ & 1.85 & $\begin{array}{l}192\left(\left(^{\text {st }} \text { cycle }\right) \text { vs. } 192\right. \\
\left(105^{\text {th }} \text { cycle }\right) \text { at } 50 \mathrm{~mA} \\
\mathrm{~g}^{-1}\end{array}$ & 150 \\
\hline 156 & & 383 & $\begin{array}{l}\mathbf{1 5 6}(40) / \mathrm{CB}(40) / \\
\text { PVDF (20) }\end{array}$ & 0.8 & $\mathrm{Na}$ & $\begin{array}{l}\text { Saturated } \mathrm{NaPF}_{6} \\
\text { in DOL/DME }\end{array}$ & 1.97 & $\begin{array}{l}162\left({ }^{\text {st }} \text { cycle }\right) \text { vs. } 149 \\
\left(150^{\text {th }} \text { cycle }\right) \text { at } 50 \mathrm{~mA} \\
\mathrm{~g}^{-1}\end{array}$ & 151 \\
\hline 157 & & 342 & $\begin{array}{l}157(40) / C B(40) / \\
\text { PVDF (20) }\end{array}$ & 0.8 & $\mathrm{Na}$ & $\begin{array}{l}\text { Saturated } \mathrm{NaPF}_{6} \\
\text { in DOL/DME }\end{array}$ & 2.01 & $\begin{array}{l}179\left(\left(^{\text {st }} \text { cycle }\right) \text { vs. } 170\right. \\
\left(150^{\text {th }} \text { cycle }\right) \text { at } 50 \mathrm{~mA} \\
\mathrm{~g}^{-1}\end{array}$ & 151 \\
\hline 158 & & 388 & $\begin{array}{l}\mathbf{1 5 8}(60) / \mathrm{KB}(30) / \\
\text { PTFE (10) }\end{array}$ & $1-2$ & $\mathrm{Na}$ & $\begin{array}{l}1 \mathrm{M} \text { NaTFSI in } \\
\mathrm{DOL} / \mathrm{DME}\end{array}$ & 2.08 & $\begin{array}{l}268\left(1^{\text {st }} \text { cycle at } 50 \mathrm{~mA}\right. \\
\left.\mathrm{g}^{-1}\right) \text { vs. } 182\left(100^{\text {th }} \text { cycle }\right. \\
\left.\text { at } 500 \mathrm{~mA} \mathrm{~g}^{-1}\right)\end{array}$ & 53 \\
\hline 159 & & 100 & $\begin{array}{l}\mathbf{1 5 9}(50) \text { / Carbon } \\
\text { fibers }(20) \text { / CB (20) } \\
\text { / PTFE (10) }\end{array}$ & & $\mathrm{Na}$ & $\begin{array}{l}1 \mathrm{M} \mathrm{NaPF}_{6} \\
\text { in EC/DEC }\end{array}$ & 3.40 & $\begin{array}{l}100\left(1^{\text {st }} \text { cycle }\right) \text { vs. } 72 \\
\left(100^{\text {th }} \text { cycle }\right) \text { at } 100 \mathrm{~mA} \\
\mathrm{~g}^{-1}\end{array}$ & 152 \\
\hline 160 & & 95 & $\begin{array}{l}\mathbf{1 6 0}(60) / \mathrm{KB}(20) / \\
\mathrm{SP}(10) / \mathrm{PTFE}(10)\end{array}$ & & $\mathrm{Na}$ & $\begin{array}{l}1 \mathrm{M} \mathrm{NaPF}_{6} \\
\text { in } \mathrm{EC} / \mathrm{DEC}\end{array}$ & 2.80 & $\begin{array}{l}133\left(1^{\text {st }} \text { cycle at } 50 \mathrm{~mA}\right. \\
\left.\mathrm{g}^{-1}\right) \text { vs. } 129\left(200^{\text {th }} \text { cycle }\right. \\
\left.\text { at } 100 \mathrm{~mA} \mathrm{~g}^{-1}\right)\end{array}$ & 153 \\
\hline 161 & & 187 & $\begin{array}{l}\mathbf{1 6 1}(65) / \mathrm{SP}(30) / \\
\operatorname{PVDF}(5)\end{array}$ & $1-2$ & $\mathrm{Na}$ & $\begin{array}{l}1 \mathrm{M} \mathrm{NaClO}_{4} \text { in } \\
\mathrm{EC} / \mathrm{DMC}\end{array}$ & 0.30 & $\begin{array}{l}207 \text { at } 0.1 \mathrm{C} \text { vs. } 117 \text { at } \\
5 \mathrm{C}\end{array}$ & 140 \\
\hline
\end{tabular}




\section{Table S3 (continued)}

\begin{tabular}{|c|c|c|c|c|c|c|c|c|c|}
\hline Number & Structure & $\begin{array}{l}\text { Specific capacity } \\
\text { b) } / \mathrm{mAh} \mathrm{g}^{-1}\end{array}$ & $\begin{array}{l}\text { Cathode } \\
\text { composition }^{\text {c) }}\end{array}$ & $\begin{array}{l}\text { AM loading } \\
\text { d) } / \mathrm{mg} \mathrm{cm}^{-2}\end{array}$ & Anode & Electrolyte & $\begin{array}{l}\text { Voltage } e^{\mathrm{e})} / \mathrm{V} \text { vs. } \\
\mathrm{Na} / \mathrm{Na}^{+}\end{array}$ & Cyclability/mAh g ${ }^{-1}$ & Ref \\
\hline 162 & & 288 & $\begin{array}{l}\mathbf{1 6 2}(25.9) / \mathrm{GO} \\
(44.1) / \mathrm{CB}(20) / \\
\mathrm{PVDF}(10)\end{array}$ & \multirow{5}{*}{1} & $\mathrm{Na}$ & $\begin{array}{l}1 \mathrm{M} \mathrm{NaClO}_{4} \text { in } \\
\mathrm{EC} / \mathrm{DMC}\end{array}$ & 1.20 & $\begin{array}{l}293\left(\left(^{\text {st }} \text { cycle }\right) \text { vs. } 117\right. \\
\left(100^{\text {th }} \text { cycle }\right) \text { at } 20 \mathrm{~mA} \\
\mathrm{~g}^{-1}\end{array}$ & 154 \\
\hline \multirow{2}{*}{163} & & \multirow{2}{*}{255} & $\begin{array}{l}\mathbf{1 6 3}(50) / \mathrm{CB}(37.5) \\
/ \mathrm{CMC}(12.5)\end{array}$ & & $\mathrm{Na}$ & $\begin{array}{l}0.8 \mathrm{M} \mathrm{NaClO}_{4} \text { in } \\
\mathrm{EC} / \mathrm{DEC}\end{array}$ & 0.30 & \multirow{2}{*}{$\begin{array}{l}310\left(1^{\text {st }} \text { cycle }\right) \text { vs. } 280 \\
\left(90^{\text {th }} \text { cycle }\right) \text { at } 30 \mathrm{~mA} \\
\mathrm{~g}^{-1} \\
192\left(1^{\text {st }} \text { cycle at } 50 \mathrm{~mA}\right. \\
\left.\mathrm{g}^{-1}\right) \text { vs. } 62\left(400^{\text {th }} \text { cycle }\right. \\
\left.\text { at } 1 \mathrm{~A} \mathrm{~g}^{-1}\right)\end{array}$} & 155 \\
\hline & & & $\begin{array}{l}\mathbf{1 6 3}(50) / \mathrm{CB}(40) / \\
\mathrm{CMC}(10)\end{array}$ & & $\mathrm{Na}$ & $1 \mathrm{M} \mathrm{NaClO}_{4}$ in $\mathrm{PC}$ & 0.18 & & 156 \\
\hline 164 & & 187 & $\begin{array}{l}\mathbf{1 6 4}(57.1) / \mathrm{SP} \\
(28.6) / \mathrm{CMC}(14.3)\end{array}$ & & $\mathrm{Na}$ & $\begin{array}{l}0.8 \mathrm{M} \mathrm{NaClO}_{4} \text { in } \\
\mathrm{EC} / \mathrm{DEC}\end{array}$ & 0.50 & $\begin{array}{l}200\left(1^{\text {st }} \text { cycle }\right) \text { vs. } 200 \\
\left(150^{\text {th }} \text { cycle }\right) \text { at } 0.1 \mathrm{C}\end{array}$ & 157 \\
\hline 165 & & 205 & $\begin{array}{l}\mathbf{1 6 5}(60) / \mathrm{CB}(33) / \\
\mathrm{CMC}(7)\end{array}$ & & $\mathrm{Na}$ & $\begin{array}{l}1 \mathrm{M} \mathrm{NaFSI} \text { in } \\
\mathrm{EC} / \mathrm{DEC}\end{array}$ & 0.25 & $\begin{array}{l}178\left({ }^{1 \mathrm{st}} \text { cycle }\right) \text { vs. } 40 \\
\left(40^{\mathrm{th}} \text { cycle }\right) \text { at } 0.025 \mathrm{C}\end{array}$ & 158 \\
\hline 166 & & 291 & $\begin{array}{l}\mathbf{1 6 6}(60) / \mathrm{CB}(30) / \\
\operatorname{PVDF}(10)\end{array}$ & & $\mathrm{Na}$ & $\begin{array}{l}1 \mathrm{M} \mathrm{NaClO}_{4} \text { in } \\
\mathrm{EC} / \mathrm{DMC}\end{array}$ & 1.20 & $\begin{array}{l}265\left(1^{\text {st }} \text { cycle at } 0.1 \mathrm{C}\right) \\
\text { vs. } 212\left(300^{\text {th }} \text { cycle at } 1\right. \\
\text { C) }\end{array}$ & 159 \\
\hline 167 & & 290 & $\begin{array}{l}\mathbf{1 6 7}(30.4) / \text { RGO } \\
(69.6)\end{array}$ & & $\mathrm{Na}$ & $\begin{array}{l}1 \mathrm{M} \mathrm{NaClO}_{4} \text { in } \\
\mathrm{EC} / \mathrm{DMC}\end{array}$ & 0.60 & $\begin{array}{l}398\left(1^{\text {st }} \text { cycle at } 50 \mathrm{~mA}\right. \\
\left.\mathrm{g}^{-1}\right) \mathrm{vs} .277\left(300^{\text {th }} \text { cycle }\right. \\
\left.\text { at } 100 \mathrm{~mA} \mathrm{~g}^{-1}\right)\end{array}$ & 160 \\
\hline 168 & & 172 & $\begin{array}{l}\mathbf{1 6 8}(50) / \mathrm{CB}(40) / \\
\mathrm{CMC}(10)\end{array}$ & 1 & $\mathrm{Na}$ & $1 \mathrm{M} \mathrm{NaClO}_{4}$ in $\mathrm{PC}$ & 0.39 & $\begin{array}{l}260\left(1^{\text {st }} \text { cycle at } 50 \mathrm{~mA}\right. \\
\left.\mathrm{g}^{-1}\right) \text { vs. } 182\left(400^{\text {th }} \text { cycle }\right. \\
\left.\text { at } 100 \mathrm{~mA} \mathrm{~g}^{-1}\right)\end{array}$ & 156 \\
\hline 169 & & 206 & $\begin{array}{l}\mathbf{1 6 9}(60) / \mathrm{SP}(30) / \\
\mathrm{PVDF}(10)\end{array}$ & & $\mathrm{Na}$ & $0.8 \mathrm{M} \mathrm{NaPF}_{6}$ in $\mathrm{PC}$ & 1.15 & $\begin{array}{l}128.9\left(1^{\text {st }} \text { cycle }\right) \text { vs. } 90 \\
\left(70^{\text {th }} \text { cycle }\right) \text { at } 0.025 \mathrm{C}\end{array}$ & 161 \\
\hline 170 & & 137 & $\begin{array}{l}\mathbf{1 7 0}(60) / \mathrm{AB}(30) / \\
\mathrm{CMC}(10)\end{array}$ & 0.6 & $\mathrm{Na}$ & $\begin{array}{l}1 \mathrm{M} \mathrm{NaPF}_{6} \text { in } \\
\mathrm{EC} / \mathrm{DMC}^{-}\end{array}$ & 0.50 & $\begin{array}{l}361\left(1^{\text {st }} \text { cycle }\right) \text { vs. } 145.8 \\
\left(140^{\text {th }} \text { cycle }\right) \text { at } 50 \mathrm{~mA} \\
\mathrm{~g}^{-1}\end{array}$ & 162 \\
\hline
\end{tabular}


Table S3 (continued)

\begin{tabular}{|c|c|c|c|c|c|c|c|c|c|}
\hline Number & Structure & $\begin{array}{l}\text { Specific } \\
\text { capacity }^{\text {b) }} / \\
\text { mAh g }^{-1}\end{array}$ & Cathode composition ${ }^{\mathrm{c}}$ & $\begin{array}{l}\text { AM loading } \\
\text { d) } / \mathrm{mg} \mathrm{cm}^{-2}\end{array}$ & Anode & Electrolyte & $\begin{array}{l}\text { Voltage }{ }^{\mathrm{e})} / \mathrm{V} \\
\text { vs. } \mathrm{Na} / \mathrm{Na}^{+}\end{array}$ & Cyclability $/ \mathrm{mAh} \mathrm{g}^{-1}$ & Ref \\
\hline 171 & & 258 & $\begin{array}{l}171(80) / \text { Carbon (15) } \\
/ \mathrm{KB}(5)\end{array}$ & $2.4-4$ & $\mathrm{Na}$ & $\begin{array}{l}1 \mathrm{M} \mathrm{NaFSI} \text { in Me- } \\
\text { THF }\end{array}$ & 0.75 & $\begin{array}{l}268\left(1^{\text {st }} \text { cycle }\right) \text { vs. } \\
261.3\left(20^{\text {th }} \text { cycle }\right) \text { at } \\
0.1 \mathrm{C}\end{array}$ & 163 \\
\hline 172 & & 120 & $\begin{array}{l}\mathbf{1 7 2}(30) / \mathrm{CB}(50) / \\
\operatorname{PVDF}(20)\end{array}$ & & $\mathrm{Na}$ & $\begin{array}{l}1 \mathrm{M} \mathrm{NaClO}_{4} \text { in } \\
\mathrm{EC} / \mathrm{DEC}\end{array}$ & 2.00 & $\begin{array}{l}150\left(\left(^{\text {st }} \text { cycle at } 70\right.\right. \\
\left.\mathrm{mA} \mathrm{g}^{-1}\right) \text { vs. } 142.5 \\
\left(500^{\text {th }} \text { cycle at } 140\right. \\
\left.\mathrm{mA} \mathrm{g}^{-1}\right)\end{array}$ & 164 \\
\hline 173 & & 225 & $\begin{array}{l}173(40) / \text { KB }(50) / \\
\text { PTFE }(10)\end{array}$ & & $\mathrm{Na}$ & $\begin{array}{l}\mathrm{NaPF}_{6} \text { in } \\
\mathrm{DOL} / \mathrm{DME}\end{array}$ & 1.80 & $\begin{array}{l}220\left(\left(^{\text {st }} \text { cycle at } 180\right.\right. \\
\left.\mathrm{mA} \mathrm{g}^{-1}\right) \text { vs. } \sim 190 \\
\left(200^{\text {th }} \text { cycle at } 1600\right. \\
\left.\mathrm{mA} \mathrm{g}^{-1}\right)\end{array}$ & 165 \\
\hline 174 & & & $\begin{array}{l}\text { 174/MC (80)/ CB (10) } \\
/ \mathrm{CMC}\end{array}$ & $1.2-1.5$ & $\mathrm{Na}$ & $\begin{array}{l}\text { THEICTA + } 1 \mathrm{M} \\
\text { NaTFSI in } \\
\text { EC/FEC }\end{array}$ & 1.25 & $\begin{array}{l}877\left(1^{\text {st }} \text { cycle }\right) \text { vs. } \\
736\left(100^{\text {th }} \text { cycle }\right) \text { at } \\
0.1 \mathrm{C}\end{array}$ & 166 \\
\hline
\end{tabular}




\section{Table S3 (continued)}

\begin{tabular}{|c|c|c|c|c|c|c|c|c|c|}
\hline Number & Structure & $\begin{array}{l}\text { Specific capacity } \\
\text { b) } / \mathrm{mAh} \mathrm{g}^{-1}\end{array}$ & Cathode composition $^{\mathrm{c})}$ & $\begin{array}{l}\text { AM } \\
\text { loading } \\
\text { d) } / \mathrm{mg} \mathrm{cm}^{-2}\end{array}$ & Anode & Electrolyte & $\begin{array}{l}\text { Voltage }^{\mathrm{e})} / \mathrm{V} \\
\text { vs. K/K }\end{array}$ & Cyclability/mAh g ${ }^{-1}$ & Ref \\
\hline \multicolumn{10}{|c|}{ Potassium-based organic battery } \\
\hline \multirow{2}{*}{175} & & \multirow{2}{*}{137} & $\begin{array}{l}175(70) / \text { SP (20) / } \\
\text { PVDF (10) }\end{array}$ & 1 & K & $\begin{array}{l}0.5 \mathrm{M} \mathrm{KPF}_{6} \text { in } \\
\mathrm{EC} / \mathrm{DEC}\end{array}$ & 2.40 & \multirow{2}{*}{$\begin{array}{l}131\left(1^{\text {st }} \text { cycle at } 10 \mathrm{~mA}\right. \\
\left.\mathrm{g}^{-1}\right) \mathrm{vs} 90\left(200^{\text {th }} \text { cycle at }\right. \\
\left.50 \mathrm{~mA} \mathrm{~g}^{-1}\right) \\
124\left(140^{\text {th }} \text { cycle at } 100 \mathrm{~mA}\right. \\
\left.\mathrm{g}^{-1}\right) \mathrm{vs}^{\circ} 98\left(1000^{\text {th }} \text { cycle at }\right. \\
\left.1000 \mathrm{~mA} \mathrm{~g}^{-1}\right)\end{array}$} & 167 \\
\hline & & & $\begin{array}{l}175(90) / \mathrm{CB}(3) / \\
\mathrm{CMC}(7)\end{array}$ & 1.2 & K & $\begin{array}{l}3 \mathrm{M} \text { KFSI in } \\
\text { DME }\end{array}$ & 2.40 & & 168 \\
\hline 176 & & 155 & $\begin{array}{l}\mathbf{1 7 6}(60) / \mathrm{CB}(30) / \mathrm{SA} \\
(10)\end{array}$ & 1.1 & K & $\begin{array}{l}0.8 \mathrm{M} \mathrm{KPF}_{6} \text { in } \\
\mathrm{EC} / \mathrm{DEC}\end{array}$ & 1.40 & $\begin{array}{l}134\left(1^{\text {st }} \text { cycle at } 0.1 \mathrm{C}\right) \text { vs. } \\
70\left(1000^{\text {th }} \text { cycle at } 1 \mathrm{C}\right)\end{array}$ & 169 \\
\hline 177 & & 220 & $\begin{array}{l}177 \text { (56) / SP (14) / AB } \\
(20) / \text { PVDF (10) }\end{array}$ & 2 & K & $\begin{array}{l}1 \mathrm{M} \mathrm{KFSI} \text { in } \\
\mathrm{EC} / \mathrm{DMC}\end{array}$ & 0.70 & $\begin{array}{l}498\left(1^{\text {st }} \text { cycle at } 0.1 \mathrm{C}\right) \text { vs. } \\
176\left(100^{\text {th }} \text { cycle at } 0.2 \mathrm{C}\right)\end{array}$ & 170 \\
\hline 178 & & & $\begin{array}{l}\mathbf{1 7 8}(70) / \mathrm{SP}(20) / \mathrm{SA} \\
(10)\end{array}$ & - & K & $\begin{array}{l}0.8 \mathrm{M} \mathrm{KPF}_{6} \text { in } \\
\mathrm{EC} / \mathrm{DEC}\end{array}$ & 1.75 & $\begin{array}{l}90\left(3^{\text {rd }} \text { cycle }\right) \text { vs. } 78\left(100^{\text {th }}\right. \\
\text { cycle }) \text { at } 0.1 \mathrm{C}\end{array}$ & 171 \\
\hline 179 & & 313.5 & $\begin{array}{l}179(54) \text { / GNTs (6) / } \\
\mathrm{AB}(30) / \mathrm{PVDF}(10)\end{array}$ & 0.53 & K & $\begin{array}{l}0.8 \mathrm{M} \mathrm{KPF}_{6} \text { in } \\
\mathrm{EC} / \mathrm{DEC}\end{array}$ & 0.70 & $\begin{array}{l}300\left(1^{\text {st }} \text { cycle }\right) \text { vs. } 222.3 \\
\left(100^{\text {th }} \text { cycle }\right) \text { at } 100 \mathrm{~mA} \mathrm{~g}^{-1}\end{array}$ & 172 \\
\hline 180 & & 225 & $\begin{array}{l}\mathbf{1 8 0}(70) \text { / SP (20) / } \\
\text { PVDF (10) }\end{array}$ & 2 & K & $\begin{array}{l}0.5 \mathrm{M} \mathrm{KTFSI} \mathrm{in} \\
\text { DOL/DME }\end{array}$ & 1.80 & $\begin{array}{l}200\left(1^{\text {st }} \text { cycle }\right) \text { vs. } 143\left(50^{\text {th }}\right. \\
\text { cycle }) \text { at } 20 \mathrm{~mA} \mathrm{~g}^{-1}\end{array}$ & 173 \\
\hline
\end{tabular}

a) The abbreviations are listed as below: AB (acetylene black), AG (activated graphene), CB (carbon black), CC (conductive carbon), CMC (sodium carboxymethyl cellulose), CMK-3 (ordered mesoporous carbon), CNT (carbon nanotube), DEC (diethyl carbonate), DMC (dimethyl carbonate), DME (dimethoxyethane), DOL (dioxolane), EC (ethylene carbonate), FEC (fuoroethylene carbonate), GNTs (graphene nanotubes), GO (graphene oxide), KB (Ketjen black), $\mathrm{KPF}_{6}$ (potassium hexafluorophosphate), KFSI [potassium bis(fluorosulfonyl)imide], KTFSI [potassium bis(fluorosulfonyl)imide], MC (mesoporous carbon), Me-THF (2Methyltetrahydrofuran), $\mathrm{NaFSI}$ [sodium bis(fluorosulfonyl)imide], $\mathrm{NaClO}_{4}$ (sodium perchlorate), $\mathrm{NaPF}_{6}$ (sodium hexafluorophosphate), NaTFSI [soudium bis(trifluoromethylsulfonil)imide], $\mathrm{PC}$ (propylene carbonate), PTFE [poly(tetrafluorethylene)], Pyr14TFSI (1-butyl-1-methylpyrrolidinium bis(trifluoromethylsulfonyl)imide), RGO (reduced graphene oxide), PVDF [poly(vinylidene fluoride)], SA (sodium alginate), TEGDME (triethylene glycol dimethyl ether), THEICTA [(S-pentaerythritol tetraacrylate-tris(2-(acryloyloxy)ethyl)) isocyanurate].

b) Theoretic specific capacity of the presented organic compound.

c) The values given in parenthesis are the weight percentage of each component in cathode.

d) The mass loading of active materials in cathode.

e) Average working voltage of the presented organic compound. 
Table S4 Properties of organic materials developed for multivalent metal batteries. ${ }^{\text {a) }}$

\begin{tabular}{|c|c|c|c|c|c|c|c|c|c|}
\hline Number & Structure & $\begin{array}{l}\text { Specific capacity } \\
/ \mathrm{mAh} \mathrm{g}^{-1}\end{array}$ & $\begin{array}{l}\text { Cathode composition } \\
\text { c) }\end{array}$ & $\begin{array}{l}\text { AM loading } \\
\text { d) } / \mathrm{mg} \mathrm{cm}^{-2}\end{array}$ & Anode & Electrolyte & $\begin{array}{l}\text { Voltage } \mathrm{e}^{\mathrm{e}} / \mathrm{V} \text { vs. } \\
\mathrm{Mg} / \mathrm{Mg}^{2+}\end{array}$ & Cyclability/mAh g ${ }^{-1}$ & Ref \\
\hline \multicolumn{10}{|c|}{ Magnesium-based organic battery } \\
\hline 181 & & 137 & $\begin{array}{l}\mathbf{1 8 1}(70) / \text { SP (20) / } \\
\text { PVDF (10) }\end{array}$ & $1.7-2.0$ & $\mathrm{Ag} / \mathrm{AgCl}$ & $\begin{array}{l}\text { 4.8 } \mathrm{M} \mathrm{Mg}\left(\mathrm{NO}_{3}\right)_{2} \text { in } \\
\mathrm{H}_{2} \mathrm{O}\end{array}$ & 2.07 & $\begin{array}{l}125\left(\left(1^{\text {st }} \text { cycle at } 20\right.\right. \\
\left.\mathrm{mA}^{-1}\right) \mathrm{vs}^{-75}\left(30^{\text {th }}\right. \\
\left.\text { at } 500 \mathrm{~mA} \mathrm{~g}^{-1}\right)\end{array}$ & 174 \\
\hline 182 & & 319 & $\begin{array}{l}\mathbf{1 8 2}(40) / \mathrm{CB}(40) / \\
\mathrm{CB}(10) / \mathrm{PVDF}(10)\end{array}$ & & $\mathrm{Mg}$ & $\begin{array}{l}0.5 \mathrm{M} \mathrm{Mg}(\mathrm{TFSI})_{2}- \\
2 \mathrm{MgCl}_{2}\end{array}$ & 2.00 & $\begin{array}{l}226\left(1^{\text {st }} \text { cycle }\right) \text { vs. } \\
74\left(30^{\text {th }} \text { cycle }\right) \text { at } 0.2 \\
\text { C }\end{array}$ & 175 \\
\hline 183 & & 388 & $\begin{array}{l}\mathbf{1 8 3}(50) / \mathrm{CB}(35) / \\
\text { PTFE (15) }\end{array}$ & & $\mathrm{Mg}$ & $\begin{array}{l}\mathrm{MgCl}_{2}-\mathrm{Mg}(\mathrm{TFSI})_{2} \text { in } \\
\text { TG/DIOX }\end{array}$ & 2.00 & $\begin{array}{l}158\left(20^{\text {th }} \text { cycle }\right) \\
\text { at } 50 \mathrm{~mA} \mathrm{~g}^{-1}\end{array}$ & 54 \\
\hline 184 & & 260 & $\begin{array}{l}\mathbf{1 8 4}(40) / \mathrm{CB}(50) / \\
\text { PVDF (10) }\end{array}$ & $1-1.5$ & $\mathrm{Mg}$ & $\begin{array}{l}0.3 \mathrm{M} \mathrm{Mg}(\mathrm{HMDS})_{2}- \\
4 \mathrm{MgCl}_{2} \text { in THF }\end{array}$ & 1.50 & $\begin{array}{l}122\left(1^{\text {st }} \text { cycle }\right) \text { vs. } \\
100.2\left(100^{\text {th }} \text { cycle }\right) \\
\text { at } 130 \mathrm{~mA} \mathrm{~g}^{-1}\end{array}$ & 176 \\
\hline 185 & & 260 & $\begin{array}{l}\mathbf{1 8 5}(40) / \mathrm{CB}(50) / \\
\mathrm{PVDF}(10)\end{array}$ & $1-1.5$ & $\mathrm{Mg}$ & $\begin{array}{l}0.3 \mathrm{M} \mathrm{Mg}(\mathrm{HMDS})_{2}- \\
4 \mathrm{MgCl}_{2} \text { in THF }\end{array}$ & 1.60 & $\begin{array}{l}132.7\left(1^{\text {st }} \text { cycle at }\right. \\
\left.130 \mathrm{~mA}^{-1}\right) \text { vs. } \\
78.7\left(1000^{\text {th }} \text { cycle at }\right. \\
\left.260 \mathrm{~mA} \mathrm{~g}^{-1}\right)\end{array}$ & 176 \\
\hline 186 & & 225 & $\begin{array}{l}\text { 186 (50) / Carbon } \\
(35) / \text { PTFE (15) }\end{array}$ & & $\begin{array}{l}\text { Mg } \\
\text { powder }\end{array}$ & $\begin{array}{l}0.37 \mathrm{M} \mathrm{MgCl}_{2} \\
+0.15 \mathrm{M} \mathrm{Mg}(\mathrm{TFSI})_{2} \\
\text { in THF/DME }\end{array}$ & 1.60 & $\begin{array}{l}225\left(1^{\text {st }} \text { cycle }\right) \text { vs. } \\
\sim 50\left(100^{\mathrm{th}} \text { cycle }\right) \text { at } \\
50 \mathrm{~mA} \mathrm{~g}^{-1}\end{array}$ & 177 \\
\hline 187 & & 54.2 & $187(60)$ / SP (40) & 1 & $\mathrm{Mg}$ & $\begin{array}{l}0.2 \mathrm{M} \mathrm{Mg}(\mathrm{TFSI})_{2} \text { in } \\
\text { Diglyme }\end{array}$ & 1.50 & $\begin{array}{l}52.8\left({ }^{\text {st }} \text { cycle at } 11\right. \\
\left.\mathrm{mA} \mathrm{g}^{-1}\right) \text { vs. } 46 \\
\left(2500^{\text {th }} \text { cycle at } 300\right. \\
\left.\mathrm{mA} \mathrm{g}^{-1}\right)\end{array}$ & 178 \\
\hline 188 & & & $\begin{array}{l}\mathbf{1 8 8} / \text { MWCNT (80) / } \\
\text { CB (10) / PTFE (10) }\end{array}$ & & $\mathrm{Ag} / \mathrm{AgCl}$ & $\begin{array}{l}4 \mathrm{M} \mathrm{Mg}(\mathrm{TFSI})_{2} \text { in } \\
\mathrm{H}_{2} \mathrm{O}\end{array}$ & 1.85 & $\begin{array}{l}130\left(1^{\text {st }} \text { cycle }\right) \text { vs. } \\
\sim 100\left(500^{\text {th }} \text { cycle }\right) \\
\text { at } 100 \mathrm{~mA} \mathrm{~g}^{-1}\end{array}$ & 179 \\
\hline
\end{tabular}




\section{Table S4 (continued)}

\begin{tabular}{|c|c|c|c|c|c|c|c|c|c|}
\hline Number & Structure & $\begin{array}{l}\text { Specific capacity }{ }^{\text {b) }} \\
\text { mAh g }^{-1}\end{array}$ & Cathode composition ${ }^{c)}$ & $\begin{array}{l}\text { AM loading } \\
\text { d) } / \mathrm{mg} \mathrm{cm}^{-2}\end{array}$ & Anode & Electrolyte & $\begin{array}{l}\text { Voltage } \mathrm{e}^{\mathrm{e}} / \mathrm{V} \\
\text { vs. } \mathrm{Mg} / \mathrm{Mg}^{2+}\end{array}$ & Cyclability $/ \mathrm{mAh} \mathrm{g}^{-1}$ & Ref \\
\hline 189 & & & $\begin{array}{l}\mathbf{1 8 9}(50) / \mathrm{SP}(40) / \\
\text { PVDF (10) }\end{array}$ & & $\mathrm{Ag} / \mathrm{AgCl}$ & $\begin{array}{l}1 \mathrm{M} \mathrm{Mg}\left(\mathrm{ClO}_{4}\right)_{2} \text { in } \\
\text { ACN }\end{array}$ & 3.20 & & 180 \\
\hline 190 & & & $\begin{array}{l}\mathbf{1 9 0}(50) / \mathrm{SP}(40) / \\
\mathrm{PVDF}(10)\end{array}$ & & $\mathrm{Ag} / \mathrm{AgCl}$ & $\begin{array}{l}1 \mathrm{M} \mathrm{Mg}\left(\mathrm{ClO}_{4}\right)_{2} \text { in } \\
\mathrm{ACN}\end{array}$ & 2.05 & & 180 \\
\hline 191 & & 203 & $\begin{array}{l}191(60) / \text { CC (30) / } \\
\text { PTFE (10) }\end{array}$ & 4 & $\mathrm{Mg}$ & $\begin{array}{l}\mathrm{Mg}(\mathrm{TFSI})_{2} \text { in } \\
\text { TEGDME/DOL }\end{array}$ & 1.75 & $\begin{array}{l}85\left(97^{\text {th }} \text { cycle }\right) \\
\text { at } 50 \mathrm{~mA} \mathrm{~g}^{-1}\end{array}$ & 181 \\
\hline
\end{tabular}




\section{Table S4 (continued)}

\begin{tabular}{|c|c|c|c|c|c|c|c|c|c|}
\hline Number & Structure & $\begin{array}{l}\text { Specific capacity } \\
\text { b) } / \mathrm{mAh} \mathrm{g}^{-1}\end{array}$ & Cathode composition ${ }^{c)}$ & $\begin{array}{l}\text { AM loading } \\
\text { d) } / \mathrm{mg} \mathrm{cm}^{-2}\end{array}$ & Anode & Electrolyte & $\begin{array}{l}\text { Voltage } e^{\mathrm{e})} / \mathrm{V} \text { vs. } \\
\mathrm{Zn} / \mathrm{Zn}^{2+}\end{array}$ & $\begin{array}{l}\text { Cyclability/mAh } \\
\mathrm{g}^{-1}\end{array}$ & Ref \\
\hline \multicolumn{10}{|c|}{ Zinc-based organic battery } \\
\hline 192 & & & $\begin{array}{l}192 \text { (60) / P-chloranil } \\
(23) \text { / CMK-3 (12) / } \\
\text { CMC (2.5) / SBR (2.5) }\end{array}$ & $3-5$ & $\mathrm{Zn}$ & $1 \mathrm{M} \mathrm{Zn}(\mathrm{OTf})_{2}$ in $\mathrm{H}_{2} \mathrm{O}$ & 1.10 & $\begin{array}{l}170\left(1^{\text {st }} \text { cycle }\right) \text { vs. } \\
90\left(100^{\text {th }} \text { cycle }\right) \text { at } \\
217 \mathrm{~mA} \mathrm{~g}^{-1}\end{array}$ & 182 \\
\hline 193 & & & $\begin{array}{l}193(60) / \text { KB (30) / } \\
\text { PTFE / (10) }\end{array}$ & $4-6$ & $\mathrm{Zn}$ & $2 \mathrm{M} \mathrm{ZnSO}_{4}$ in $\mathrm{H}_{2} \mathrm{O}$ & 0.75 & $\begin{array}{l}336\left(\left(^{\text {st }} \text { cycle at } 40\right.\right. \\
\left.\mathrm{mA} \mathrm{g}^{-1}\right) \text { vs. } 145 \\
\left(1000^{\text {th }} \text { cycle at }\right. \\
\left.3000 \mathrm{~mA} \mathrm{~g}^{-1}\right)\end{array}$ & 183 \\
\hline 194 & & & $\begin{array}{l}194(60) / \text { SP (35) / } \\
\text { PVDF (5) }\end{array}$ & 2.5 & $\mathrm{Zn}$ & $3 \mathrm{M} \mathrm{Zn}(\mathrm{OTf})_{2}$ & 1.00 & $\begin{array}{l}335\left(\left(^{\text {st }} \text { cycle at } 20\right.\right. \\
\left.\mathrm{mA} \mathrm{g}^{-1}\right) \text { vs. } \sim 130 \\
\left(1000^{\text {th }} \text { cycle at } 500\right. \\
\left.\mathrm{mA} \mathrm{g}^{-1}\right)\end{array}$ & 184 \\
\hline 195 & & 388 & $\begin{array}{l}195(60) / C C(30) / \\
\text { PVDF (10) }\end{array}$ & & $\mathrm{Zn}$ & $3 \mathrm{M} \mathrm{Zn}(\mathrm{OTf})_{2}$ in $\mathrm{H}_{2} \mathrm{O}$ & 0.90 & $\begin{array}{l}203\left(1^{\text {st }} \text { cycle at } 0.1\right. \\
\text { C) vs. } 175\left(80^{\text {th }}\right. \\
\text { cycle at } 0.2 \mathrm{C})\end{array}$ & 185 \\
\hline 196 & & 133 & $\begin{array}{l}196(20) / M W C N T \\
\text { (70) / PVDF (10) }\end{array}$ & 1.5 & $\mathrm{Zn}$ & $1 \mathrm{M} \mathrm{Zn}\left(\mathrm{BF}_{4}\right)_{2}$ in $\mathrm{H}_{2} \mathrm{O}$ & 1.10 & $\begin{array}{l}128\left(1000^{\text {th }} \text { cycle }\right) \\
\text { at } 120 \mathrm{C}\end{array}$ & 186 \\
\hline 197 & & & 197 / Carbon felts & 1.5 & $\mathrm{Zn}$ & $1 \mathrm{M} \mathrm{Zn}(\mathrm{OTf})_{2}$ in $\mathrm{H}_{2} \mathrm{O}$ & 1.10 & $\begin{array}{l}200\left(\left(^{\text {st }} \text { cycle at } 50\right.\right. \\
\left.\mathrm{mA} \mathrm{g}^{-1}\right) \text { vs. } 145 \\
\left(200^{\text {th }} \text { cycle at } 5000\right. \\
\left.\mathrm{mA} \mathrm{g}^{-1}\right)\end{array}$ & 187 \\
\hline
\end{tabular}




\section{Table S4 (continued)}

\begin{tabular}{|c|c|c|c|c|c|c|c|c|c|}
\hline Number & Structure & $\begin{array}{l}\text { Specific capacity } \\
\text { b) } / \mathrm{mAh} \mathrm{g}^{-1}\end{array}$ & $\begin{array}{l}\text { Cathode composition } \\
\text { c) }\end{array}$ & $\begin{array}{l}\text { AM loading } \\
\text { d) } / \mathrm{mg} \mathrm{cm}^{-2}\end{array}$ & Anode & Electrolyte & $\begin{array}{l}\text { Voltage }{ }^{\mathrm{e})} / \mathrm{V} \\
\text { vs. } \mathrm{Ca} / \mathrm{Ca}^{2+}\end{array}$ & $\begin{array}{l}\text { Cyclability/mAh } \\
\mathrm{g}^{-1}\end{array}$ & Ref \\
\hline \multicolumn{10}{|c|}{ Calcium-based organic battery } \\
\hline 198 & & 137 & Activated carbon & & $\begin{array}{l}\mathbf{1 9 8}(70) / \mathrm{SP} \\
(20) / \mathrm{PVDF} \\
(10)\end{array}$ & $\begin{array}{l}\text { Saturated } \mathrm{Ca}\left(\mathrm{NO}_{3}\right)_{2} \text { in } \\
\mathrm{H}_{2} \mathrm{O}\end{array}$ & 2.40 & $\begin{array}{l}87\left(1^{\text {st }} \text { cycle at } 20\right. \\
\left.\mathrm{mA} \mathrm{g}^{-1}\right)\end{array}$ & 174 \\
\hline 199 & & 183 & $\begin{array}{l}199(60) / \text { SP }(20) / \\
\text { PTFE (10) }\end{array}$ & & $\mathrm{Ag} / \mathrm{AgCl}$ & $\begin{array}{l}2.5 \mathrm{M} \mathrm{Ca}\left(\mathrm{NO}_{3}\right)_{2} \text { in } \\
\mathrm{H}_{2} \mathrm{O}\end{array}$ & 2.80 & $\begin{array}{l}130\left(1^{\text {st }} \text { cycle }\right) \\
\text { vs. } 105\left(4000^{\text {th }}\right. \\
\text { cycle }) \text { at } 925 \mathrm{~mA} \\
\mathrm{~g}^{-1}\end{array}$ & 188 \\
\hline
\end{tabular}




\section{Table S4 (continued)}

\begin{tabular}{|c|c|c|c|c|c|c|c|c|c|}
\hline Number & Structure & $\begin{array}{l}\text { Specific capacity }{ }^{\text {b) }} \\
/ \mathrm{mAh} \mathrm{g}^{-1}\end{array}$ & $\begin{array}{l}\text { Cathode } \\
\text { composition }^{\mathrm{c}}\end{array}$ & $\begin{array}{l}\text { AM loading } \\
\text { d) } / \mathrm{mg} \mathrm{cm}^{-2}\end{array}$ & Anode & Electrolyte & $\begin{array}{l}\text { Voltage }^{\mathrm{e})} / \mathrm{V} \\
\text { vs. Al/ } \mathrm{Al}^{3+}\end{array}$ & $\begin{array}{l}\text { Cyclability/mAh } \\
\mathrm{g}^{-1}\end{array}$ & Ref \\
\hline \multicolumn{10}{|c|}{ Aluminum-based organic battery } \\
\hline 200 & & 140 & $\begin{array}{l}\mathbf{2 0 0}(30) \text { / Denka } \\
\text { black (50) / PVDF } \\
(20)\end{array}$ & 0.5 & $\mathrm{Al}$ & $\mathrm{AlCl}_{3} / \mathrm{EMIC}$ & 1.30 & $\begin{array}{l}94\left(1^{\text {st }} \text { cycle }\right) \\
\text { vs. } 53\left(5000^{\text {th }}\right. \\
\text { cycle }) \text { at } 2 \mathrm{~mA} \mathrm{~g}^{-1}\end{array}$ & 189 \\
\hline 201 & & 99.9 & $\begin{array}{l}201(60) \text { / Kynar / } \\
\text { SP (30) / DP (30) }\end{array}$ & & $\mathrm{Al}$ & $\mathrm{AlCl}_{3} / \mathrm{EMIC}$ & 1.20 & $\begin{array}{l}\sim 50\left(1^{\text {st }} \text { cycle }\right) \text { at } \\
0.2 \mathrm{C}\end{array}$ & 190 \\
\hline 202 & & 79.6 & $\begin{array}{l}\mathbf{2 0 2}(60) \text { / Kynar } \\
\text { SP (30) / DP (30) }\end{array}$ & & $\mathrm{Al}$ & $\mathrm{AlCl}_{3} / \mathrm{EMIC}$ & 1.30 & $\begin{array}{l}\sim 80\left(1^{\text {st }} \text { cycle }\right) \text { at } \\
0.2 \mathrm{C}\end{array}$ & 190 \\
\hline 203 & & 133 & $\begin{array}{l}\mathbf{2 0 3}(50) / \mathrm{CB}(40) / \\
\text { PVDF (10) }\end{array}$ & 0.5 & $\mathrm{Al}$ & $\mathrm{AlCl}_{3} / \mathrm{EMIC}$ & 1.70 & $\begin{array}{l}100\left(1000^{\text {th }} \text { cycle }\right) \\
\text { at } 200 \mathrm{~mA} \mathrm{~g}^{-1}\end{array}$ & 191 \\
\hline
\end{tabular}

a) The abbreviations are listed as below: $\mathrm{ACN}$ (acetonitrile), $\mathrm{AlCl}_{3}$ (aluminum chloride), $\mathrm{Ca}\left(\mathrm{NO}_{3}\right)_{2}$ (calcium nitrate), $\mathrm{CB}$ (carbon black), $\mathrm{CC}$ (conductive carbon), $\mathrm{CMC}$ (sodium carboxymethyl cellulose), $\mathrm{CMK}-3$ (ordered mesoporous carbon), DIOX (diethylene dioxide), DOL (dioxolane), DP (dibutyl phthalate), EMIC (1-ethyl-3-methylimidazolium tetrachloroaluminate), KB (Ketjen black), Me-THF (2-Methyltetrahydrofuran), $\mathrm{MgCl}_{2}$ (magnesium chloride), $\mathrm{Mg}\left(\mathrm{ClO}_{4}\right)_{2}$ (magnesium perchlorate), $\mathrm{Mg}\left(\mathrm{NO}_{3}\right)_{2}$ (magnesium nitrate), $\mathrm{Mg}(\mathrm{HMDS})_{2}$ [magnesium bis(hexamethyldisilazide)], Mg(TFSI) $)_{2}$ [magnesium bis(trifluoromethylsulfonil)imide], MWCNT (multiwalled carbon nanotubes), PTFE [poly(tetrafluorethylene)], PVDF [poly(vinylidene fluoride)], TEGDME (triethylene glycol dimethyl ether), THF (tetrahydrofuran), TG (tetraglyme), Zn(BF $)_{2}$ (zinc tetrafluoroborate), $\mathrm{Zn}(\mathrm{OTf})_{2}$ (zinc trifluoromethanesulfonate), $\mathrm{ZnSO}_{4}$ (zinc sulfate).

b) Theoretical specific capacity of the presented organic compound.

c) The values given in parenthesis are the weight percentage of each component in cathode.

d) The mass loading of active materials in cathode.

e) Average working voltage of the presented organic compound. 


\section{Enlarged figures for the state-of-art on organic batteries}

(a)

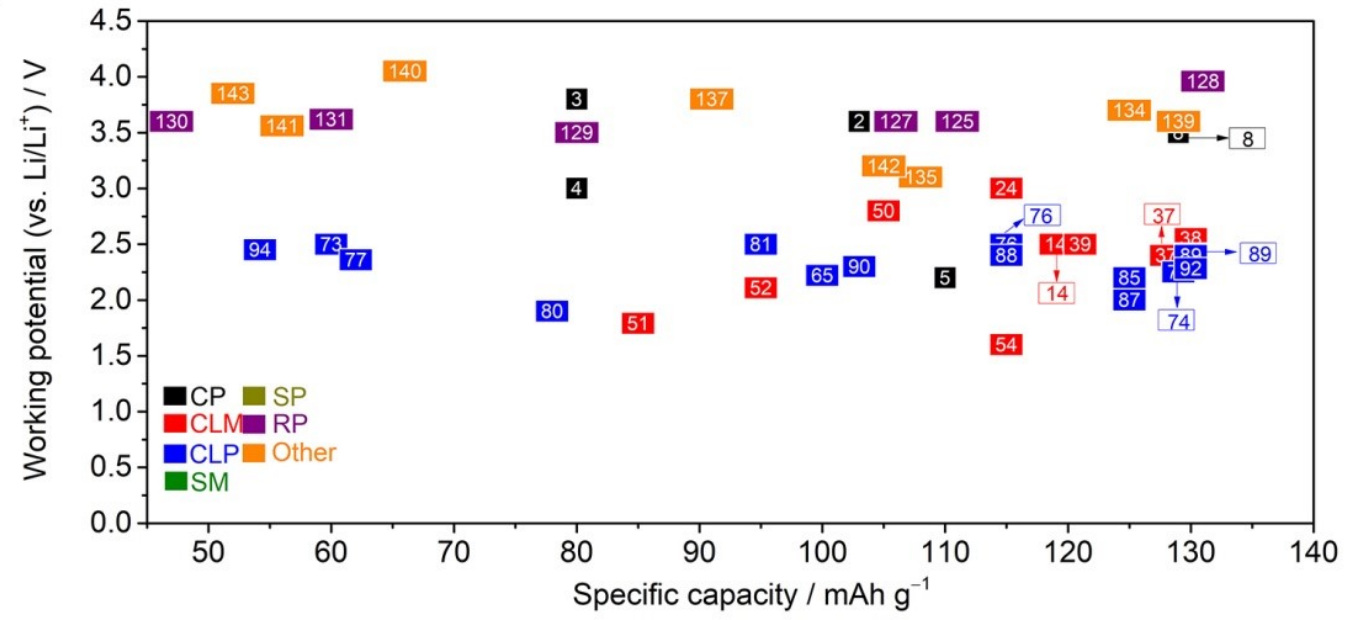

(b)

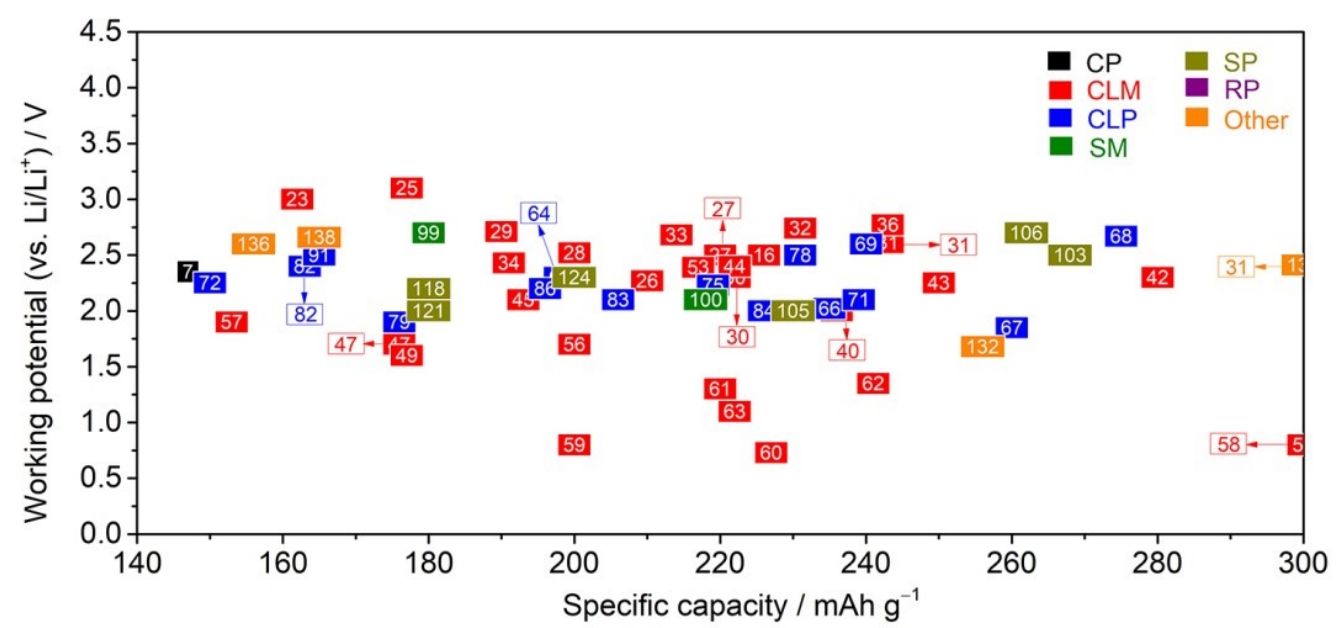

(c)

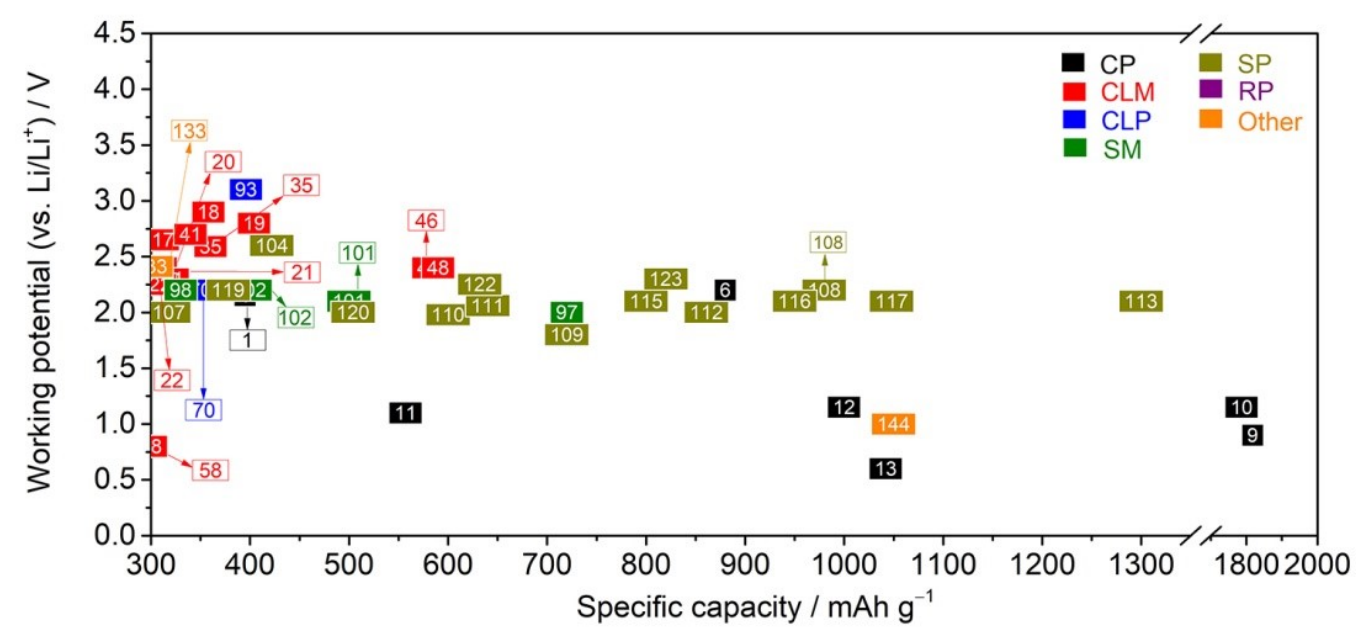

Figure S1. Enlarged version of Figure 1a: (a) from 40 to $140 \mathrm{mAh} \mathrm{g}^{-1}$; (b) from 140 to $300 \mathrm{mAh} \mathrm{g}^{-1}$; and (c) from 300 to $2000 \mathrm{mAh} \mathrm{g}^{-1}$. 


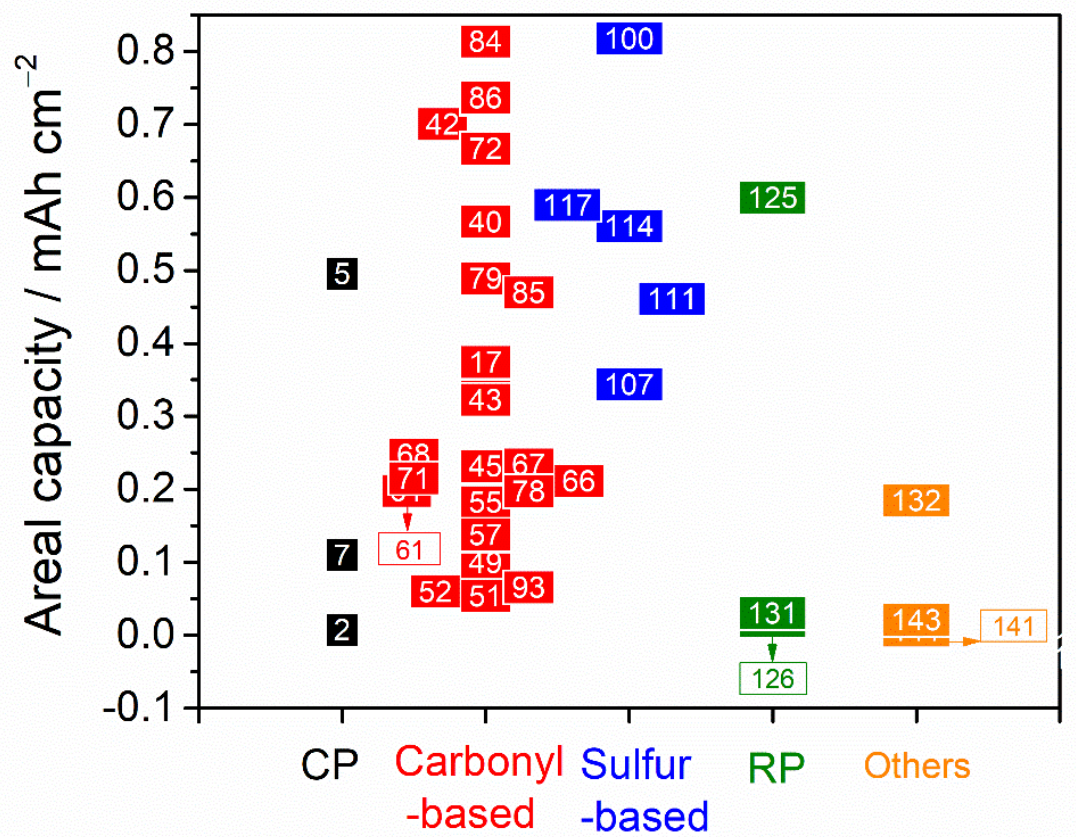

Figure S2. Enlarged version of Figure 1c from 0 to $0.9 \mathrm{mAh} \mathrm{cm}^{-2}$. 


\section{Energy density calculations details}

Realistic calculations of energy density at cell level were performed based on the model previously described by our group ${ }^{190}$ with the help of Scilab software, a free open-source numerically oriented programming language. A set of parameters including specific capacity and working potential of active material, electrode composition or electrolyte amount were considered for estimating the energy density of organic batteries. The details of the Scilab codes used for each calculation are shown in Section 3 in Supporting Information. Table S5 presents the properties of some typical organic materials used for the calculations. These materials were selected from an in-depth study of the state-of-art work on organic batteries as displayed in Tables S2-S4. For comparison, the energy density of conventional lithium-ion (herewith $\mathrm{LiFePO}_{4}, \mathrm{LFP}$ ) and lithium-sulfur batteries were calculated, and the corresponding parameters obtained from representative publications are also presented in Table S5. ${ }^{193-194}$

Table S5. Properties of the different active material families used for the calculations. ${ }^{\text {a) }}$

\begin{tabular}{|c|c|c|c|c|c|c|}
\hline & & & Abbreviation & Capacity / $\mathrm{mAh} \mathrm{g}^{-1}$ & $\begin{array}{l}\text { Discharge voltage } \\
\text { / V vs. } \mathrm{Li} / \mathrm{Li}^{+}\end{array}$ & $\begin{array}{l}\text { Density / } \\
\mathrm{g} \mathrm{cm}^{-3}\end{array}$ \\
\hline \multirow{2}{*}{$\begin{array}{l}\text { Conventional } \\
\text { batteries }\end{array}$} & \multicolumn{2}{|c|}{ Intercalation, $\mathrm{LiFePO}_{4}$} & LFP & 160 & 3.4 & 3.65 \\
\hline & \multicolumn{2}{|c|}{ Sulfur } & $\mathrm{S}$ & 900 & 2.1 & 1.96 \\
\hline \multirow{6}{*}{$\begin{array}{l}\text { Organic } \\
\text { batteries }\end{array}$} & \multicolumn{2}{|c|}{ Conjugated polymer } & $\mathrm{CP}$ & 85 & 3 & 1.2 \\
\hline & \multirow{2}{*}{$\begin{array}{l}\text { Carbonyl } \\
\text { based }\end{array}$} & Small molecules & CLM & 400 & 2.3 & 1.2 \\
\hline & & Polymer & CLP & 225 & 2.2 & 1.2 \\
\hline & \multirow{2}{*}{$\begin{array}{l}\text { Sulfur } \\
\text { containing }\end{array}$} & Small molecules & SM & 428 & 2.2 & 1.5 \\
\hline & & Polymer & SP & 800 & 2.1 & 1.5 \\
\hline & \multicolumn{2}{|c|}{ Radical polymer } & $\mathrm{RP}$ & 110 & 3.6 & 1.2 \\
\hline
\end{tabular}

a) The reference compounds for each material family are $\mathrm{LiFePO}_{4}$ (LFP), elemental sulfur (S), poly(indole) (CP), $p$-benzoquinone (CLM), poly(anthraquinonyl sulfide) (CLP), diphenyl trisulfide (SM), sulfur-rich side chain anchored in thiourea aldehyde (SP) and 2,2,6,6-tetranethylpiperidinyloxy-4-yl acrylamide (RP)

Energy density calculations were carried out for liquid and polymer electrolyte based systems. Table S6 shows specific parameters related to each cell component, i.e., positive electrode, electrolyte and negative electrode. For different families of organic materials, positive electrode recipe was adjusted to realistic values, according to stateof-art research shown in Tables S2-S4. For liquid electrolytes, state-of-art poly(vinylidene difluoride) binder and salt-containing ethylene carbonate/dimethyl 
carbonate electrolytes were selected. For solid polymer electrolytes, a poly(ethylene oxide) electrolyte was selected, which acts both as binder and catholyte in the positive electrode. In addition, the solid polymer electrolyte works simultaneously as ion conductive material and physical barrier between electrodes, avoiding the use of any extra separator. In the negative electrode, the use of self-standing metallic lithium allows its direct use without any additional binder, conductive carbon or current collector.

Table S6. Properties of the electrodes and the electrolyte for different electrolyte systems.

\begin{tabular}{|c|c|c|c|}
\hline \multicolumn{2}{|l|}{ Parameters } & Liquid electrolyte configuration & $\begin{array}{l}\text { Polymer electrolyte } \\
\text { configuration }\end{array}$ \\
\hline \multirow{8}{*}{$\begin{array}{l}\text { Positive } \\
\text { electrode }\end{array}$} & Active material / wt $\%$ & $96 / 75 / 70$ & $75 / 50 / 50$ \\
\hline & Binder $/ \mathrm{wt} \%$ & $2 / 10 / 10$ & $20 / 35 / 30$ \\
\hline & Binder density $/ \mathrm{g} \mathrm{cm}^{-3}$ & 1.76 & 1.20 \\
\hline & Carbon $/ \mathrm{wt} \%$ & $2 / 15 / 20$ & $4 / 15 / 20$ \\
\hline & Carbon density $/ \mathrm{g} \mathrm{cm}^{-3}$ & 2.2 & 2.2 \\
\hline & Cathode porosity / \% & 10 & 20 \\
\hline & Current collector mass $/ \mathrm{mg} \mathrm{cm}^{-2}$ & 2.7 & 2.7 \\
\hline & Current collector thickness / $\mu \mathrm{m}$ & 10 & 10 \\
\hline \multirow{5}{*}{ Electrolyte } & Separator mass $/ \mathrm{mg} \mathrm{cm}^{-2}$ & 1.2 & N.A. \\
\hline & Separator thickness / $\mu \mathrm{m}$ & 25 & N.A. \\
\hline & $\begin{array}{l}\text { Electrolyte / Active material ratio } \\
(\mathrm{E} / \mathrm{AM})\left(\mu \mathrm{L} \mathrm{mg}^{-1}\right)\end{array}$ & 1 & N.A. \\
\hline & Electrolyte thickness / $\mu \mathrm{m}$ & N.A. & 30 \\
\hline & Electrolyte porosity / \% & N.A. & 20 \\
\hline $\begin{array}{l}\text { Negative } \\
\text { electrode }\end{array}$ & $\begin{array}{l}\text { Negative / positive electrode } \\
\text { capacity ratio }\end{array}$ & 3 & 3 \\
\hline
\end{tabular}

Some modifications of the previous parameters were done for different parameter effect studies, which are clearly indicated in the each description.

For the development of the Rangone plot, four material families were selected and calculations were done based on liquid electrolyte cells with a fixed areal capacity of 1 $\mathrm{mAh} \mathrm{cm}^{-2}$. Reported capacity values for different discharge rates were extracted from various references and are displayed in Table S7. First, gravimetric energy at each rate was calculated based on discharge capacity. Later, power density was calculated by the division of gravimetric energy by discharge time. 
Table S7. Properties of the electrodes and the electrolyte for different electrolyte systems.

\begin{tabular}{|c|c|c|c|c|c|c|c|c|c|c|c|c|c|c|c|c|c|}
\hline \multirow[b]{2}{*}{ Material. } & \multicolumn{17}{|c|}{ Capacity $/ \mathrm{mAh} \mathrm{g}^{-1}$} \\
\hline & $0.1 \mathrm{C}$ & $0.2 \mathrm{C}$ & $0.5 \mathrm{C}$ & $1 \mathrm{C}$ & $2 \mathrm{C}$ & $5 \mathrm{C}$ & $10 \mathrm{C}$ & $20 \mathrm{C}$ & $40 \mathrm{C}$ & $50 \mathrm{C}$ & $60 \mathrm{C}$ & $80 \mathrm{C}$ & $100 \mathrm{C}$ & $120 \mathrm{C}$ & $200 \mathrm{C}$ & $300 \mathrm{C}$ & Ref. \\
\hline LFP & 160 & 160 & 157 & 150 & 140 & 125 & 105 & 80 & 50 & & & & & & & & $195-198$ \\
\hline CLP & 225 & 200 & 180 & 170 & 160 & & 150 & 140 & & 122 & & & 90 & & 60 & & 51,199 \\
\hline $\mathrm{SC}$ & 1285 & 1176 & 956 & 850 & 750 & 500 & $250^{\text {a) }}$ & & & & & & & & & & 103 \\
\hline RP & 110 & 110 & 110 & 110 & 110 & 110 & 110 & 98 & 88 & & 83 & 77 & 72 & 70 & & 50 & $114,117,200$ \\
\hline
\end{tabular}

a) Estimated value based on capacity decay due to the absence of reported data. 
Calculations for cells based on alternative negative electrodes were carried in two polymeric carbonyl-based materials [i.e., poly(anthraquinonyl sulfide) (PAQS) and poly(3,4,9,10-perylenetetracarboxylic dianhydride) (PTCDA)]. Assuming the same reaction mechanism occurs in each metal-ion battery, the working voltage of each cell could be deducted from the difference of the redox potential for other metals vs. the value for $\mathrm{Li} / \mathrm{Li}^{+}$redox pair. Calculations were performed based on liquid electrolyte systems at fixed areal capacities of 1 and $2 \mathrm{mAh} \mathrm{cm}^{-2}$ and necessary parameters for these calculations are listed in Table S8.

Table S8. Properties of the negative and positive electrode materials.

\begin{tabular}{|c|c|c|c|c|c|}
\hline & & Capacity/mAh g ${ }^{-1}$ & $\begin{array}{l}\text { Discharge } \\
\text { voltage/V vs. } \\
\mathrm{Li} / \mathrm{Li}^{+}\end{array}$ & $\begin{array}{l}\text { Depositing } \\
\text { potential/V vs. } \\
\mathrm{Li} / \mathrm{Li}^{+}\end{array}$ & Density $/ \mathrm{g} \mathrm{cm}^{-3}$ \\
\hline \multirow{2}{*}{$\begin{array}{l}\text { Positive electrode } \\
\text { material }\end{array}$} & PAQS & 225 & 2.2 & & 1.20 \\
\hline & PTCDA & 145 & 2.4 & & 1.20 \\
\hline \multirow{6}{*}{$\begin{array}{l}\text { Negative } \\
\text { electrode } \\
\text { material }^{201-202}\end{array}$} & $\mathrm{Li}$ & 3861 & & 0.00 & 0.53 \\
\hline & $\mathrm{Ca}$ & 1337 & & 0.18 & 1.55 \\
\hline & $\mathrm{Na}$ & 1166 & & 0.34 & 0.97 \\
\hline & $\mathrm{K}$ & 685 & & 0.12 & 0.89 \\
\hline & $\mathrm{Mg}$ & 2205 & & 0.67 & 1.74 \\
\hline & $\mathrm{Al}$ & 2980 & & 1.38 & 2.70 \\
\hline
\end{tabular}




\section{Scilab codes for energy density calculation}

//Energy Density Assessment of Organic Batteries

//Xabier Judez, Lixin Qiao, Michel Armand and Heng Zhang

(hzhang@cicenergigune.com)

//Electrical Energy Storage Department, CIC Energigune, Parque Tecnológico de Àlava, Albert Einstein 48, 01510 Miñano, Álava, Spain

clc, clear, tic()

//Common parameters

$\mathrm{mcc}=2.7 * 10^{\wedge}-3 / / \mathrm{g} / \mathrm{cm} 2$. Current collector mass

$\mathrm{vcc}=10^{*} 10^{\wedge}-4 / / \mathrm{cm} 3 / \mathrm{cm} 2$ Current collector thickness

$\mathrm{NP}=3 / /$ Negative/positivie capacity ratio

$\mathrm{CLi}=3861 / / \mathrm{mAh} / \mathrm{g}$ Lithium theoretical capacity

rhoLi $=0.53 / / \mathrm{g} / \mathrm{cm} 3$. Lithium density

rhoC=2.2 ///g/cm3. Carbon density

//Active material parameters

AMvec=["LiIC LCO" "Li-IC LFP" "S" "Conjugated" "Carbonyl, PAQS" "Carbonyl, Poly(imide)" "S Disulfide" "S copolymer" "S thioether" "Radical" "Carbonyl, lawsone" "Disulfide, dip tris"]

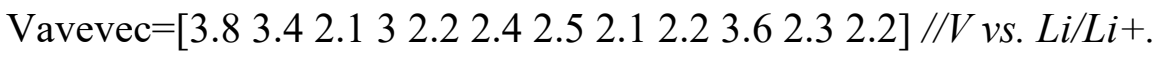

Cgvec=[140 16090084225150350800300110400 428] //mAh/g. Specific capacity

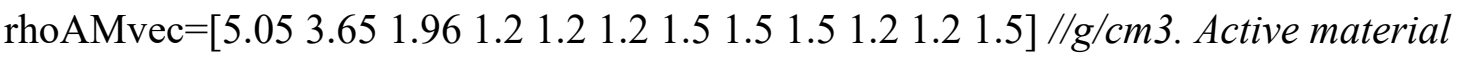
density 


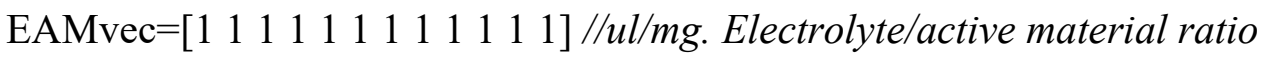

Cavec $=(0: 0.1: 5) / / m A h \mathrm{~cm}-2$. Studied areal capacities

FigNu=1//Figure number

///General functions

function $[\mathbf{E g}]=\underline{\text { GravimetricEnergy }}(\mathbf{C a}) / /$ Gravimetric energy

$\mathrm{mAM}=\mathbf{C a} / \mathrm{Cg} / / \mathrm{gAM} / \mathrm{cm} 2$. active mass

mcatho=mAM/AMperc $/ / \mathrm{g} / \mathrm{cm} 2$. Total cathode mass

$\mathrm{mLi}=\mathbf{C a} * \mathrm{NP} / \mathrm{CLi} / / \mathrm{g} / \mathrm{cm} 2$. Li mass

Wtot $=$ mcc + mcatho $+\mathrm{mLi}+$ msep + melec $/ / \mathrm{g} / \mathrm{cm} 2$. Total mass

$\mathbf{E g}=$ Vave* $\mathbf{C a} /$ Wtot $/ / W h / k g$

endfunction

function $[\mathbf{E v}]=$ VolumetricEnergy $(\mathbf{C a}) / /$ Volumetric Energy

$\mathrm{mAM}=\mathbf{C a} / \mathrm{Cg} / / \mathrm{gAM} / \mathrm{cm} 2$ active mass

vcatho $=(\mathrm{mAM} / \mathrm{rhoAM}+\mathrm{mAM} *$ Cperc $/ \mathrm{AMperc} / \mathrm{rhoC}+\mathrm{mAM} *$ Bperc/AMperc $/ \mathrm{rhoB}) /(1-$

cathoporosity) $/ / \mathrm{cm} 3 / \mathrm{cm} 2$. Cathode volume

$\mathrm{vLi}=\mathbf{C a} * \mathrm{NP} / \mathrm{CLi} / \mathrm{rhoLi} / / / / \mathrm{cm} 3 / \mathrm{cm} 2$. Lithium volume

$\mathrm{vtot}=\mathrm{vcc}+\mathrm{vcatho}+\mathrm{vLi}+\mathrm{vsep}+\mathrm{velec} / / \mathrm{cm} 3 / \mathrm{cm} 2$. Total volume

$\mathbf{E v}=$ Vave $^{*} \mathbf{C a} /$ vtot $/ / W h / l$

endfunction 
|IIII/I/Liquid system//II

//Composition for each material

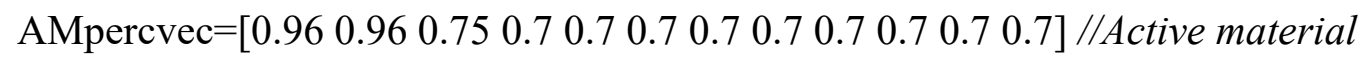
fraction

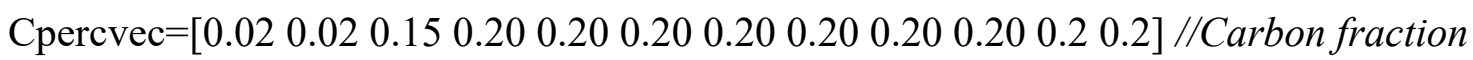

/liquid system properties

$\mathrm{rhoB}=1.76 / / \mathrm{g} / \mathrm{cm} 3$. Binder density

cathoporosity=0.1//Cathode porosity.

msep $=1.2 * 10^{\wedge}-3 / / g$ cm 2 . Separator mass

vsep $=25^{*} 10^{\wedge}-4 / / \mathrm{cm} 3 / \mathrm{cm} 2$. Separator volume

rhoelec $=1.13 / / \mathrm{g} / \mathrm{cm} 3$. Electrolyte density

//Calculation

$[a \mathrm{~b}]=\operatorname{size}(\mathrm{AMvec})$

for $i=1: b$

$$
\begin{aligned}
& \text { Vave=Vavevec(i) } \\
& \text { Cg=Cgvec(i) } \\
& \text { rhoAM=rhoAMvec(i) } \\
& \text { AMperc=AMpercvec(i) } \\
& \text { Cperc=Cpercvec(i) }
\end{aligned}
$$


Bperc=1-AMperc-Cperc //Binder fraction

EAM=EAMvec(i)

for $\mathrm{j}=1$ :length(Cavec)

$\mathrm{Ca}=\operatorname{Cavec}(\mathrm{j})$

melec $=\mathrm{Ca} / \mathrm{Cg} * 1000 * \mathrm{EAM} / 1000 *$ rhoelec $/ / \mathrm{g} / \mathrm{cm} 2$. Electrolyte mass

$\mathrm{velec}=0 / / \mathrm{cm} 3 / \mathrm{cm} 2$. Electrolyte volume. No extra volume, electrolyte fills the

separator

$\operatorname{EgLiq}(\mathrm{j}, \mathrm{i})=$ GravimetricEnergy $(\mathrm{Ca})$

EvLiq(j,i) $=$ VolumetricEnergy $(\mathrm{Ca})$

end

end

//Figures

$\underline{\operatorname{scf}}(\mathrm{FigNu})$

$\underline{\text { subplot }}(1,2,1)$

plot2d(Cavec,EgLiq)

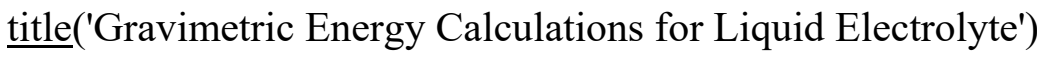

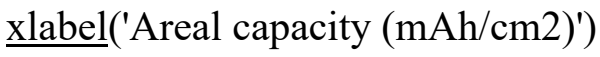

ylabel('Gravimetric energy (Wh / kg)')

legend('Active material = '+string $(\mathrm{AMvec}))$

subplot $(1,2,2)$

plot2d(Cavec,EvLiq)

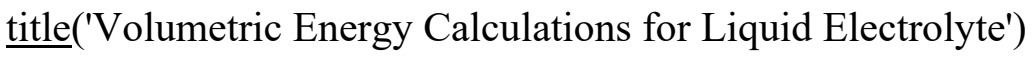

xlabel('Areal capacity $(\mathrm{mAh} / \mathrm{cm} 2)$ ')

ylabel('Volumetric energy (Wh / L)') 
$\mathrm{FigNu}=\mathrm{FigNu}+1 / /$ Figure number

///////Polymer system////

//Composition for each material

AMpercvec $=\left[\begin{array}{llllllllllll}0.75 & 0.75 & 0.50 & 0.50 & 0.50 & 0.50 & 0.50 & 0.50 & 0.50 & 0.50 & 0.50 & 0.50\end{array}\right]$

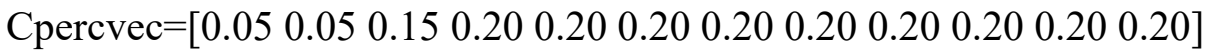

//Polymer system properties

rhoB $=1.2$

cathoporosity $=0.2$

msep $=0 / / \mathrm{g} / \mathrm{cm} 2$. Separator mass. No additional separator

vsep $=0 / / \mathrm{cm} 3 / \mathrm{cm} 2$. Separator volume. No additional separator

rhoelec $=\mathrm{rhoB} / / \mathrm{g} / \mathrm{cm} 3$. The electrolyte acts a binder and viceversa

Thick= 30 //um. Electrolyte thickness

eelec $=0.2$ // Electrolyte porosity

//Calculation

$[\mathrm{a} b]=\operatorname{size}(\mathrm{AMvec})$

for $\mathrm{i}=1: \mathrm{b}$

$$
\begin{aligned}
& \text { Vave=Vavevec }(i) \\
& \mathrm{Cg}=\text { Cgvec(i) } \\
& \text { rhoAM=rhoAMvec(i) } \\
& \text { AMperc=AMpercvec(i) } \\
& \text { Cperc=Cpercvec(i) }
\end{aligned}
$$




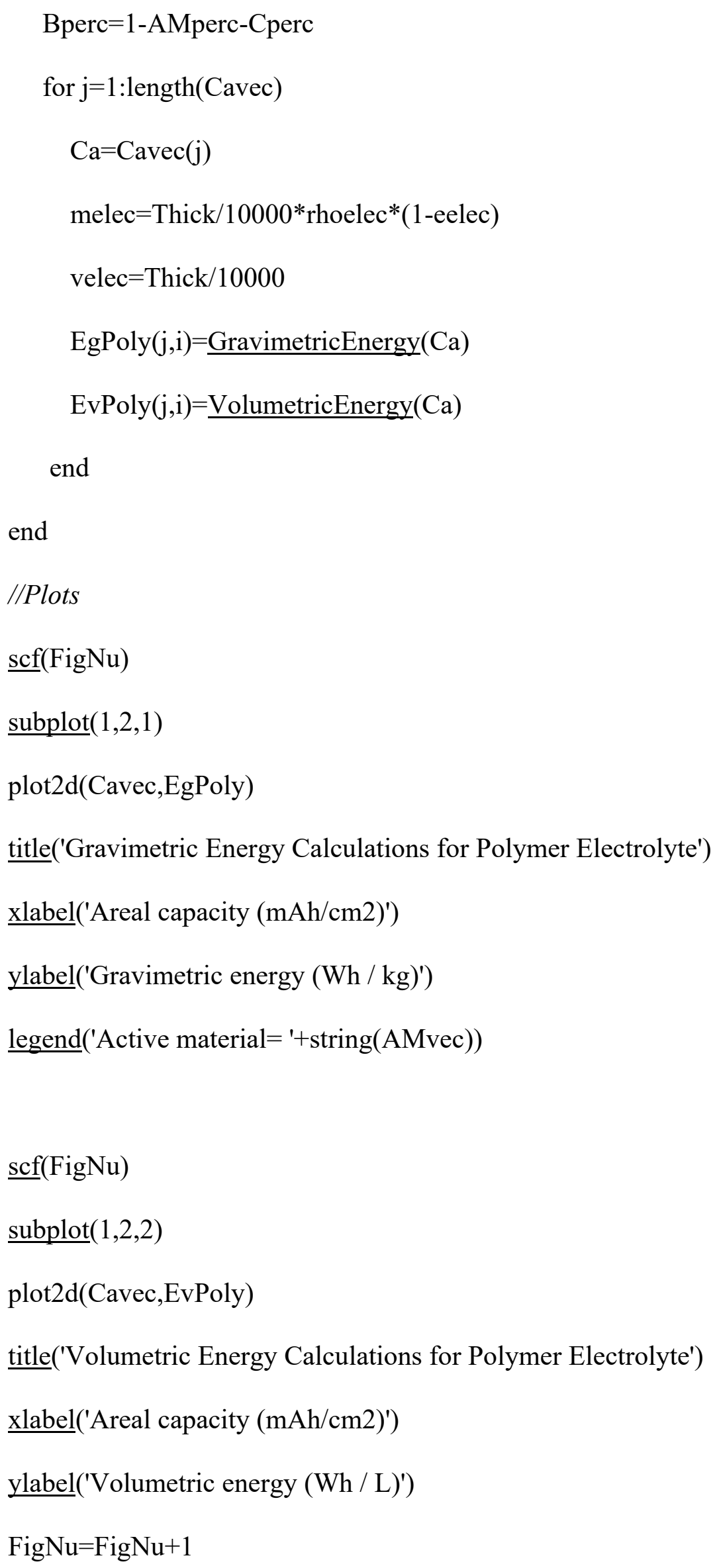




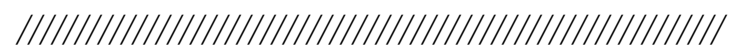

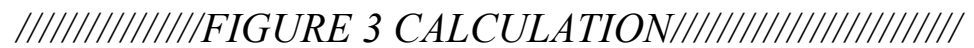

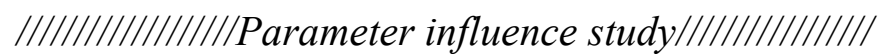

//Selected active material's parameters

AMvec=["Carbonyl Lawsome" "Carbonyl PAQS" "Scopolymer" "Radical"]

Vavevec $=\left[\begin{array}{llll}2.3 & 2.2 & 2.1 & 3.6\end{array}\right]$

Cgvec $=\left[\begin{array}{llll}400 & 225 & 800 & 110\end{array}\right]$

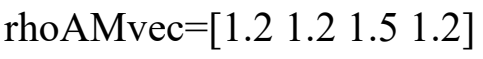

$\mathrm{EAMvec}=\left[\begin{array}{llll}1 & 1 & 1 & 1\end{array}\right]$

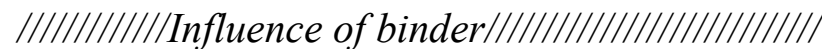

Cperc $=[0.2] / /$ Fixed carbon percent

Bpercvec $=[0.2,0.1,0] / /$ Different binder percentages

//Liquid

rhoB $=1.76 ;$ cathoporosity $=0.1 ; \mathrm{msep}=1.2 * 10^{\wedge}-3 ; \mathrm{vsep}=25^{*} 10^{\wedge}-4 ;$ rhoelec $=1.13$

//Calculation

$[\mathrm{a} \mathrm{b}]=\operatorname{size}(\mathrm{AMvec})$

for $\mathrm{i}=1: \mathrm{b}$
$\mathrm{AM}=\mathrm{AMvec}(\mathrm{i})$
Vave $=$ Vavevec $(i)$
$\mathrm{Cg}=\operatorname{Cgvec}(\mathrm{i})$
rhoAM=rhoAMvec(i) 


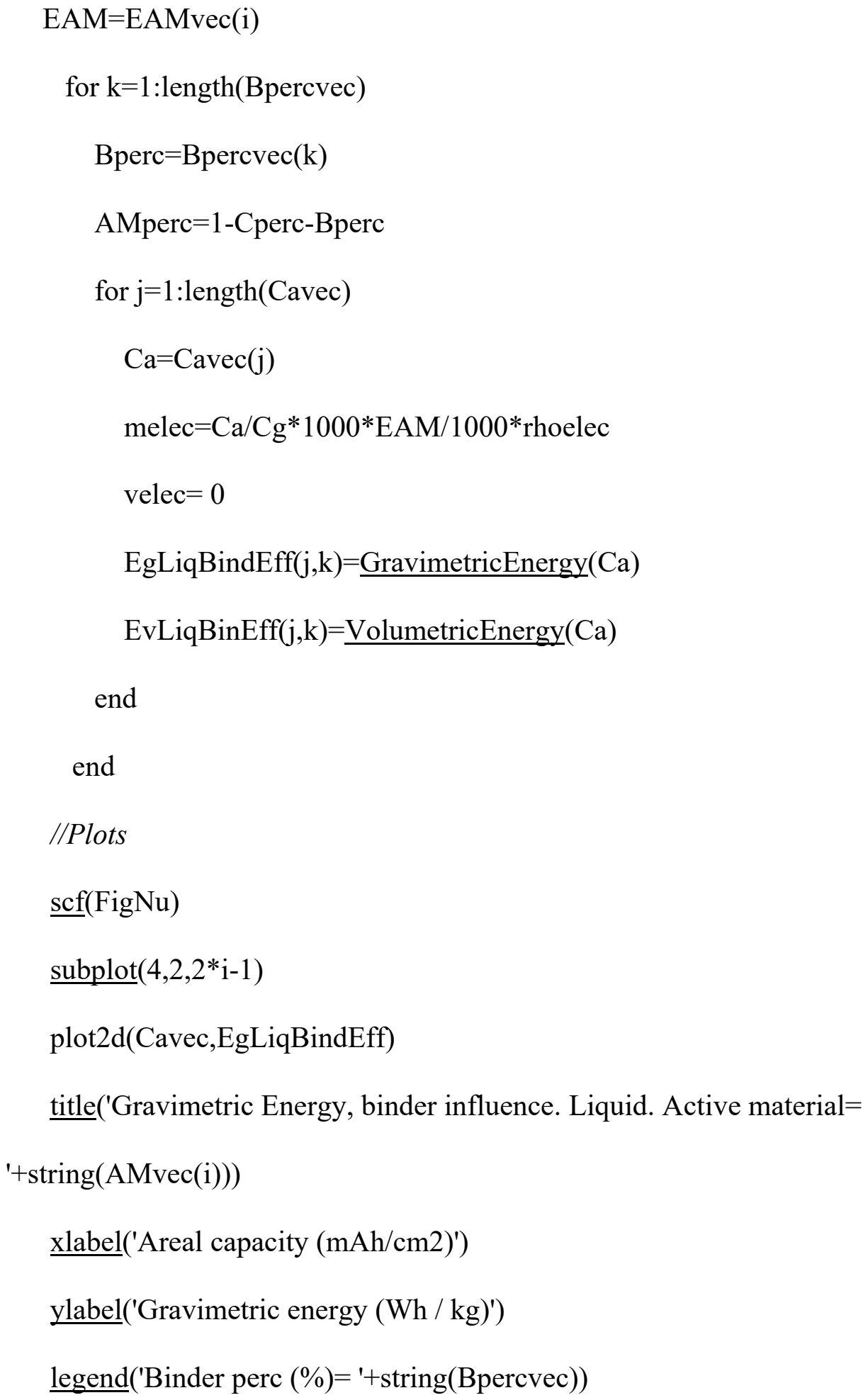




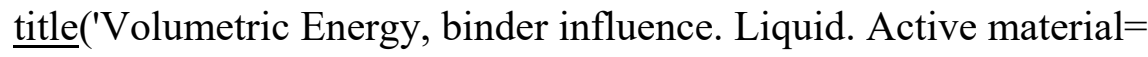

'+string(AMvec(i)))

xlabel('Areal capacity $(\mathrm{mAh} / \mathrm{cm} 2)$ ')

ylabel('Volumetric energy (Wh / L)')

end

$\mathrm{FigNu}=\mathrm{FigNu}+1$

//Polymer

Cperc $=[0.2] / /$ Fixed carbon content

Bpercvec $=[0.4,0.3,0.2] / /$ Different binder percentages

$\mathrm{rhoB}=1.2 ;$ cathoporosity $=0.2 ; \mathrm{msep}=0 ; \mathrm{vsep}=0 ;$ rhoelec $=$ rhoB; Thick $=30 ;$ eelec $=0.2$

$[a \mathrm{~b}]=\operatorname{size}($ AMvec $)$

for $\mathrm{i}=1: \mathrm{b}$

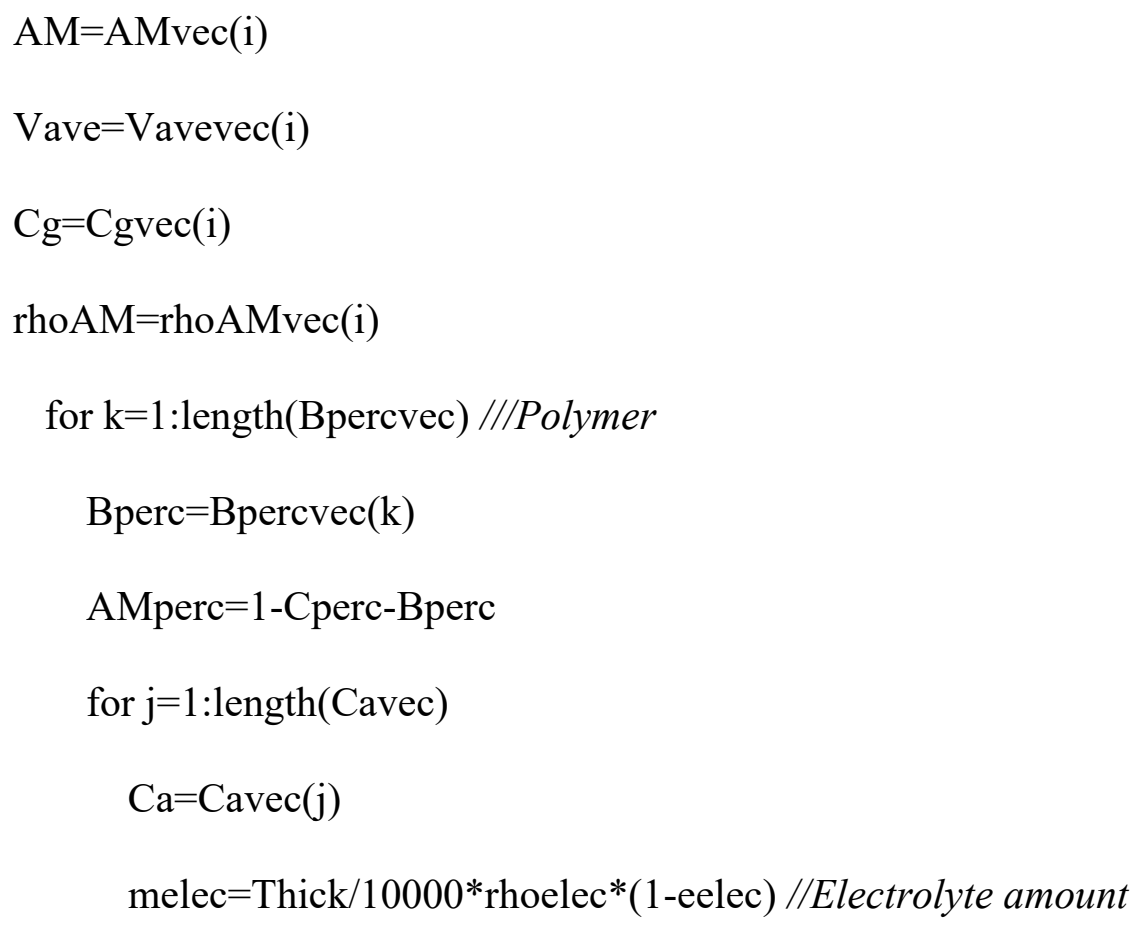




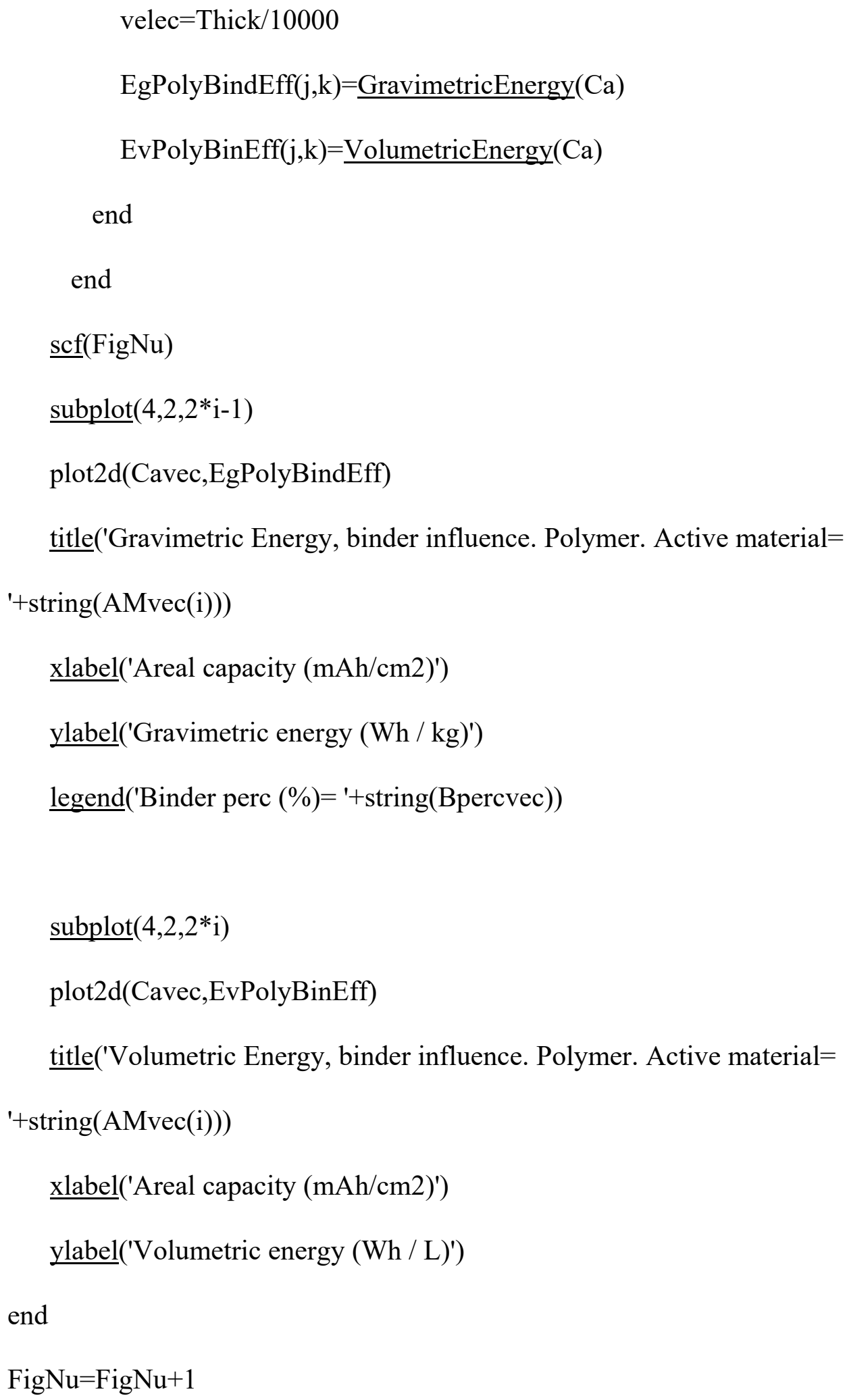


Cpercvec $=[0.40,0.20,0.05]$

//Liquid

$\mathrm{rhoB}=1.76 ;$ cathoporosity $=0.1 ; \mathrm{msep}=1.2^{*} 10^{\wedge}-3 ; \mathrm{vsep}=25^{*} 10^{\wedge}-4 ;$ rhoelec $=1.13$

Bperc $=0.1 / /$ Fixed binder percentage

//Calculation

$[a \mathrm{~b}]=\operatorname{size}(\mathrm{AMvec})$

for $i=1: b$

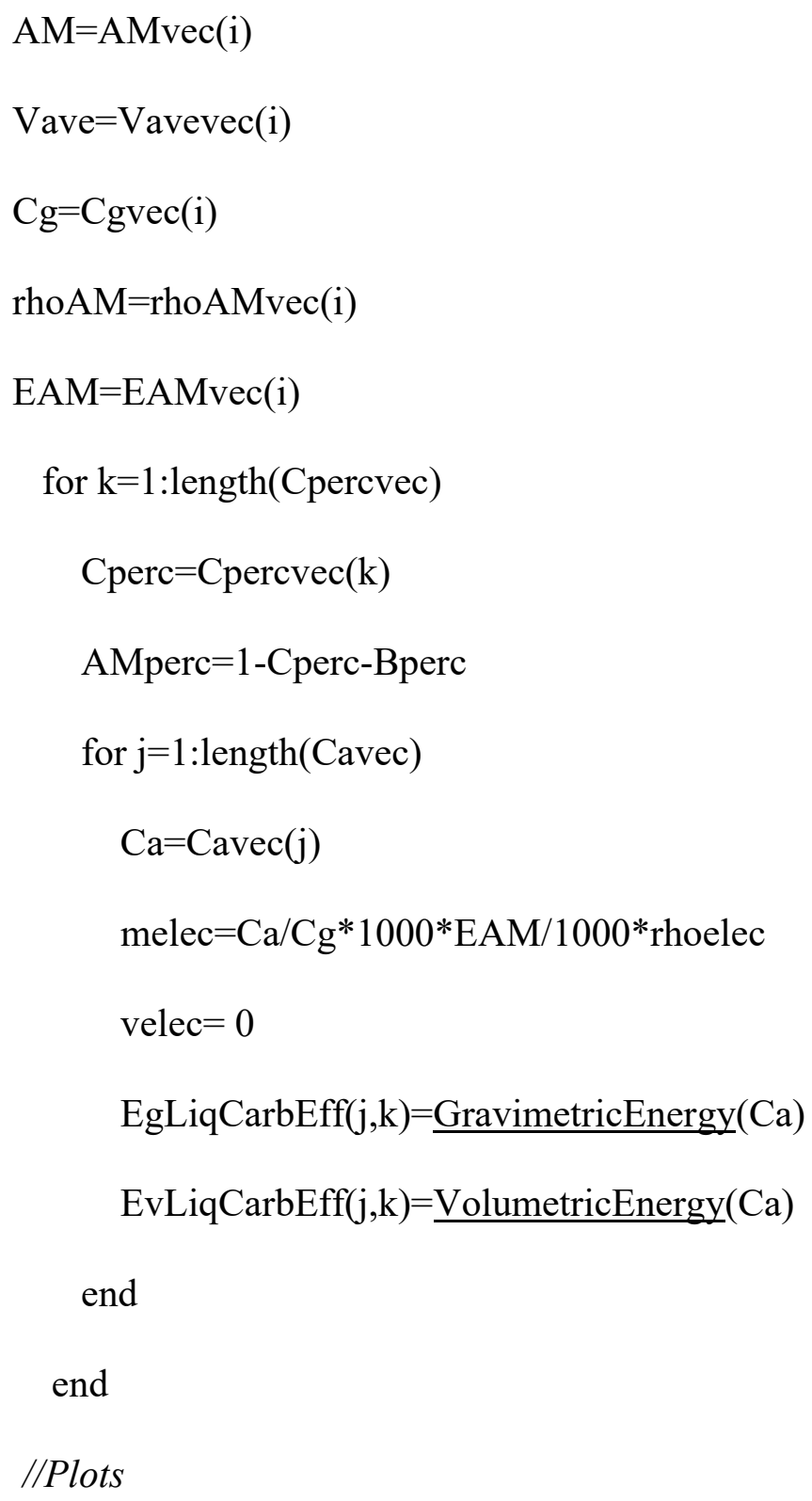


$\underline{\operatorname{scf}}(\mathrm{FigNu})$

$\underline{\operatorname{subplot}}(4,2,2 * \mathrm{i}-1)$

plot2d(Cavec,EgLiqCarbEff)

$\underline{\text { title}}$ ('Gravimetric Energy, carbon influence. Liquid. Active material=

'+string(AMvec(i)))

xlabel('Areal capacity $(\mathrm{mAh} / \mathrm{cm} 2)$ ')

ylabel('Gravimetric energy (Wh / kg)')

legend $('$ Carbon perc $(\%)=$ '+string $($ Cpercvec $))$

$\underline{\text { subplot }}(4,2,2 * \mathrm{i})$

plot2d(Cavec,EvLiqCarbEff)

$\underline{\text { title}}($ 'Volumetric Energy, carbon influence. Liquid. Active material=

'+string(AMvec(i)))

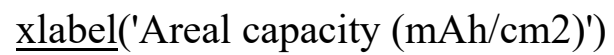

ylabel('Volumetric energy (Wh / L)')

end

$\mathrm{FigNu}=\mathrm{FigNu}+1$

//Polymer

rhoB $=1.2 ;$ cathoporosity $=0.2 ; \mathrm{msep}=0 ; \mathrm{vsep}=0 ;$ rhoelec $=$ rhoB; Thick $=30 ;$ eelec $=0.2$

Bperc $=0.3 / /$ Fixed binder percentage

//Calculation

$[a \mathrm{~b}]=\operatorname{size}(\mathrm{AMvec})$

for $\mathrm{i}=1: \mathrm{b}$ 


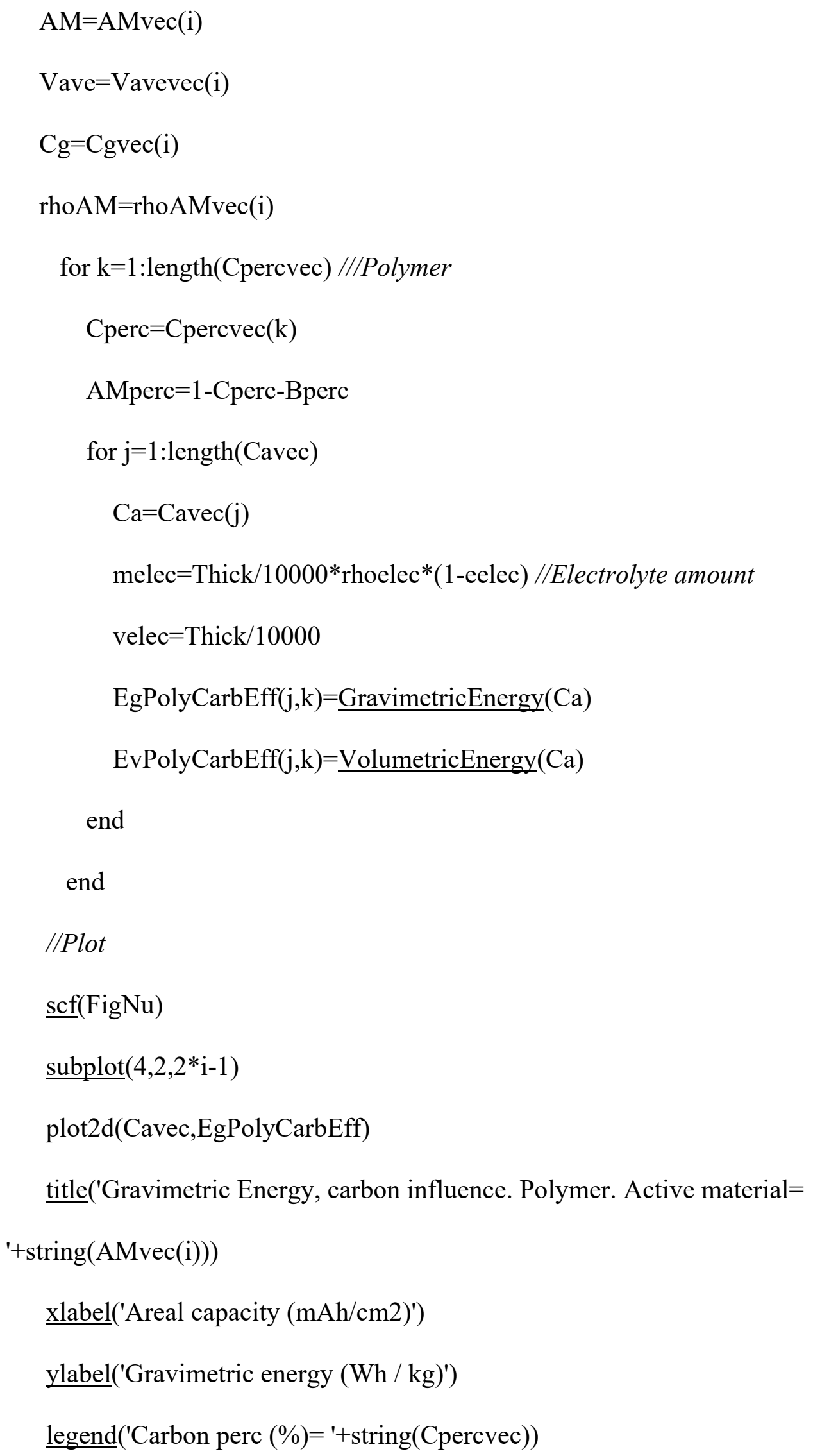


$\underline{\text { subplot }}(4,2,2 * \mathrm{i})$

plot2d(Cavec,EvPolyCarbEff)

title('Volumetric Energy, carbon influence. Polymer. Active material= '+string(AMvec(i)))

xlabel('Areal capacity $\left.(\mathrm{mAh} / \mathrm{cm} 2)^{\prime}\right)$

ylabel('Volumetric energy (Wh / L)')

end

$\mathrm{FigNu}=\mathrm{FigNu}+1$

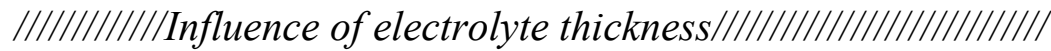

Thickvec $=\left[\begin{array}{lll}30 & 50 & 100\end{array}\right]$

AMpercvec $=\left[\begin{array}{llll}0.50 & 0.50 & 0.50 & 0.50\end{array}\right]$

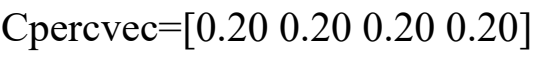

$\mathrm{rhoB}=1.2 ;$ cathoporosity $=0.2 ; \mathrm{msep}=0 ; \mathrm{vsep}=0 ;$ rhoelec $=$ rhoB; Thick $=30 ;$ eelec $=0.2$

//Calculation

$[a \mathrm{~b}]=\operatorname{size}(\mathrm{AMvec})$

for $\mathrm{i}=1: \mathrm{b}$

$$
\begin{aligned}
& \mathrm{AM}=\mathrm{AMvec}(\mathrm{i}) \\
& \text { Vave=Vavevec(i) } \\
& \mathrm{Cg}=\mathrm{Cgvec}(\mathrm{i}) \\
& \text { rhoAM=rhoAMvec(i) }
\end{aligned}
$$

AMperc=AMpercvec(i) 


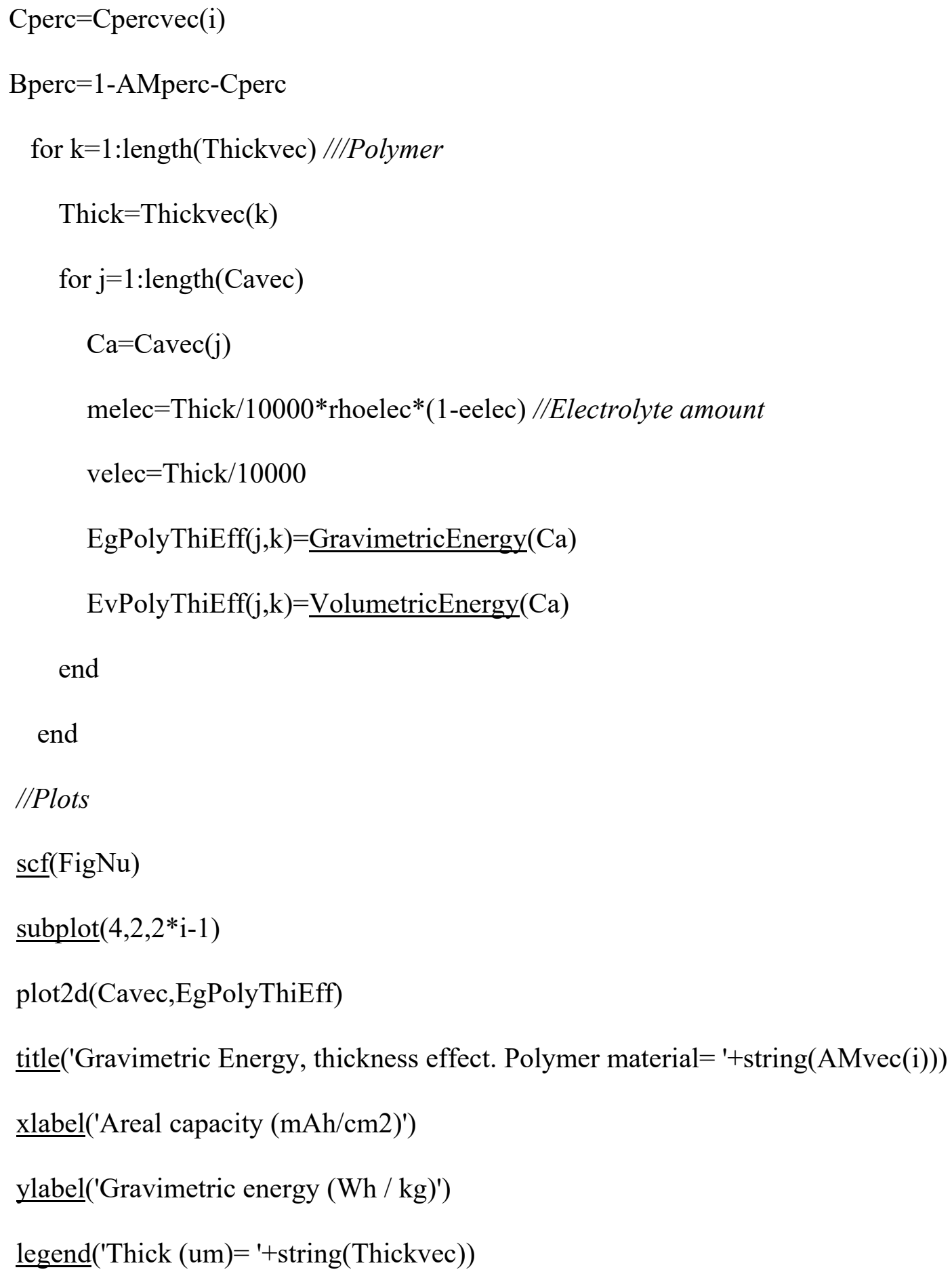


ylabel('Volumetric energy (Wh / L)')

end

$\mathrm{FigNu}=\mathrm{FigNu}+1$

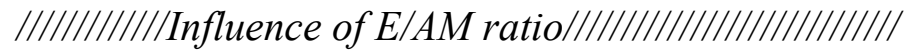

EAMvec $=[1,3,10]$

AMpercvec $=\left[\begin{array}{llll}0.70 & 0.70 & 0.70 & 0.70\end{array}\right]$

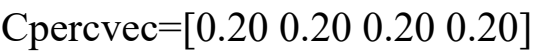

$\mathrm{rhoB}=1.76 ;$ cathoporosity $=0.1 ; \mathrm{msep}=1.2 * 10^{\wedge}-3 ; \mathrm{vsep}=25^{*} 10^{\wedge}-4 ;$ rhoelec $=1.13$

//Calculation

$[a \mathrm{~b}]=\operatorname{size}(\mathrm{AMvec})$

for $\mathrm{i}=1: \mathrm{b}$

$$
\begin{aligned}
& \text { AM=AMvec(i) } \\
& \text { Vave=Vavevec(i) } \\
& \mathrm{Cg}=\text { Cgvec(i) } \\
& \text { rhoAM=rhoAMvec(i) } \\
& \text { AMperc=AMpercvec(i) } \\
& \text { Cperc=Cpercvec(i) } \\
& \text { Bperc }=1-A M p e r c-C p e r c \\
& \text { for } \mathrm{k}=1: \text { length(EAMvec) } \\
& \text { EAM=EAMvec(k) } \\
& \text { for } \mathrm{j}=1 \text { :length(Cavec) }
\end{aligned}
$$




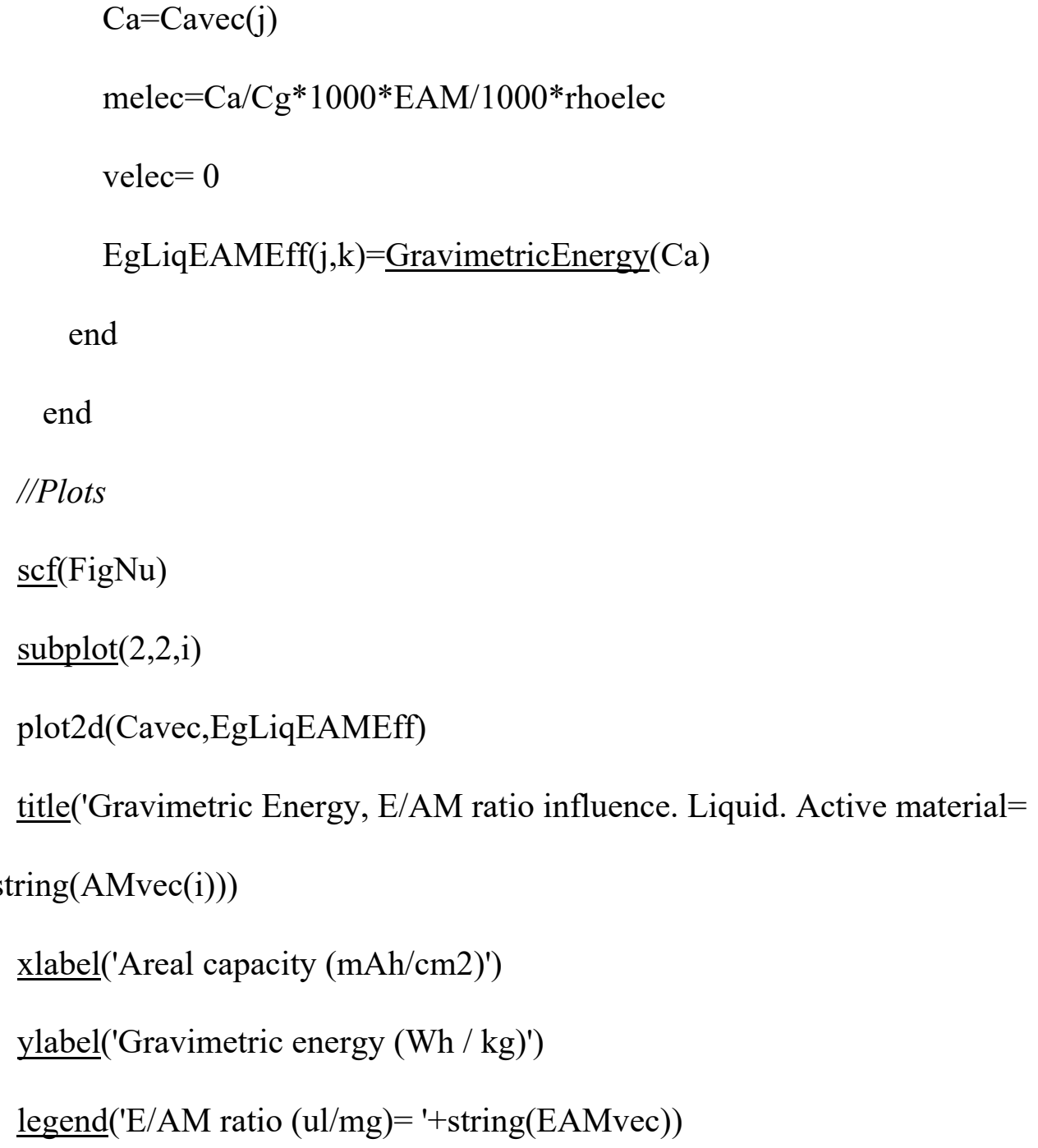

\section{//Liquid}

AMpercvec $=\left[\begin{array}{llll}0.70 & 0.70 & 0.70 & 0.70\end{array}\right]$

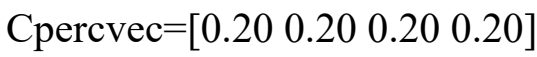




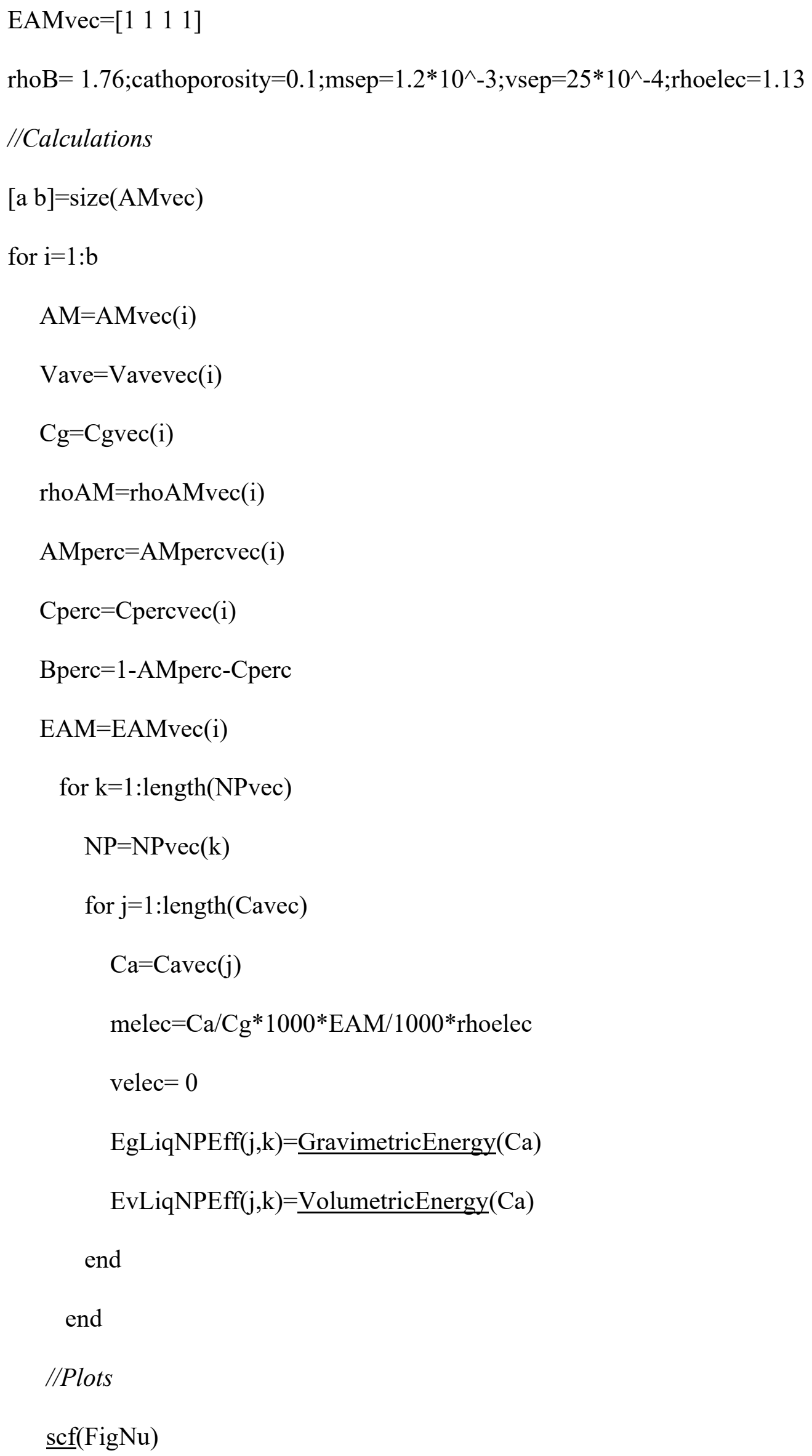


$\underline{\text { subplot }}(4,2,2 * \mathrm{i}-1)$

plot2d(Cavec,EgLiqNPEff)

title('Gravimetric Energy, N/P ratio influence. Liquid. Active material= '+string(AMvec(i)))

xlabel('Areal capacity $\left.(\mathrm{mAh} / \mathrm{cm} 2)^{\prime}\right)$

ylabel('Gravimetric energy $\left.(\mathrm{Wh} / \mathrm{kg})^{\prime}\right)$

legend $(' \mathrm{~N} / \mathrm{P}$ ratio $=$ '+string $(\mathrm{NPvec}))$

$\underline{\operatorname{subplot}}(4,2,2 * \mathrm{i})$

plot2d(Cavec,EvLiqNPEff)

$\underline{\text { title}(' G r a v i m e t r i c ~ E n e r g y, ~ N / P ~ r a t i o ~ i n f l u e n c e . ~ L i q u i d . ~ A c t i v e ~ m a t e r i a l=~}$

'+string(AMvec(i)))

xlabel('Areal capacity $(\mathrm{mAh} / \mathrm{cm} 2)$ ')

ylabel('Gravimetric energy (Wh / kg)')

legend('N/P ratio= '+string(NPvec))

end

$\mathrm{FigNu}=\mathrm{FigNu}+1$

//Polymer

AMpercvec $=\left[\begin{array}{llll}0.50 & 0.50 & 0.50 & 0.50\end{array}\right]$

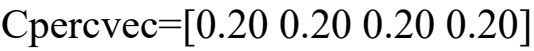

rhoB $=1.2 ;$ cathoporosity $=0.2 ; \mathrm{msep}=0 ; \mathrm{vsep}=0 ;$ rhoelec $=$ rhoB; Thick $=30 ;$ eelec $=0.2$

$[a \mathrm{~b}]=\operatorname{size}(\mathrm{AMvec})$

for $\mathrm{i}=1: \mathrm{b}$ 


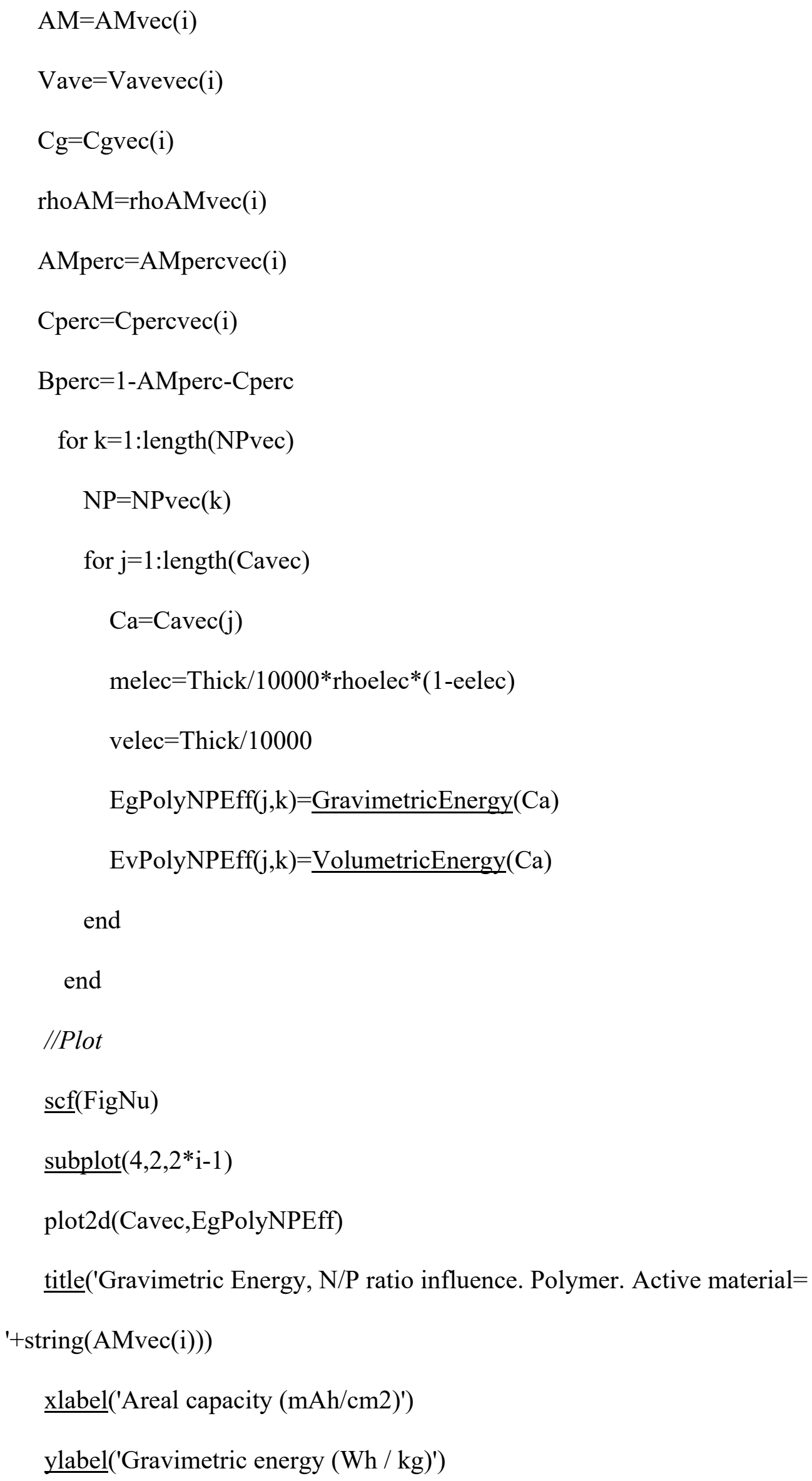


legend $(' \mathrm{~N} / \mathrm{P}$ ratio $=$ '+string(NPvec) $)$

$\underline{\operatorname{subplot}}(4,2,2 * \mathrm{i})$

plot2d(Cavec,EvPolyNPEff)

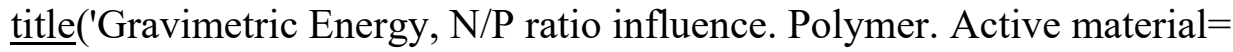

'+string(AMvec(i)))

xlabel('Areal capacity $(\mathrm{mAh} / \mathrm{cm} 2)$ ')

ylabel('Gravimetric energy $\left.(\mathrm{Wh} / \mathrm{kg})^{\prime}\right)$

end

$\mathrm{FigNu}=\mathrm{FigNu}+1$

- ||||||||||||||||||||||||||||||||||||||||||||||||||||||||||||

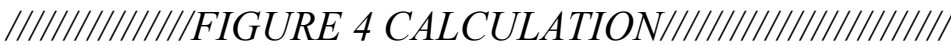

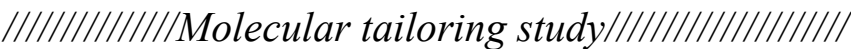

$\mathrm{AM}=["$ Carbonyl FAMILY"]

rhoAM=[1.2]

|/I/I/I/II/I/I/I/I///Constant voltage, different capacity.

Cgvec $=(200: 1: 400) / /$ Varied capacity

Vave $=2.2 / /$ Fixed voltage

$\mathrm{Ca}=1$

$\mathrm{NP}=3$

//Calculation 


\section{//Liquid}

$\mathrm{AMperc}=0.70, \mathrm{Cperc}=0.20, \mathrm{EAM}=1, \mathrm{rhoB}=1.76 ;$ cathoporosity $=0.1 ; \mathrm{msep}=1.2^{*} 10^{\wedge} \_$ $3 ; \mathrm{vsep}=25^{*} 10^{\wedge}-4 ;$ rhoelec $=1.13$

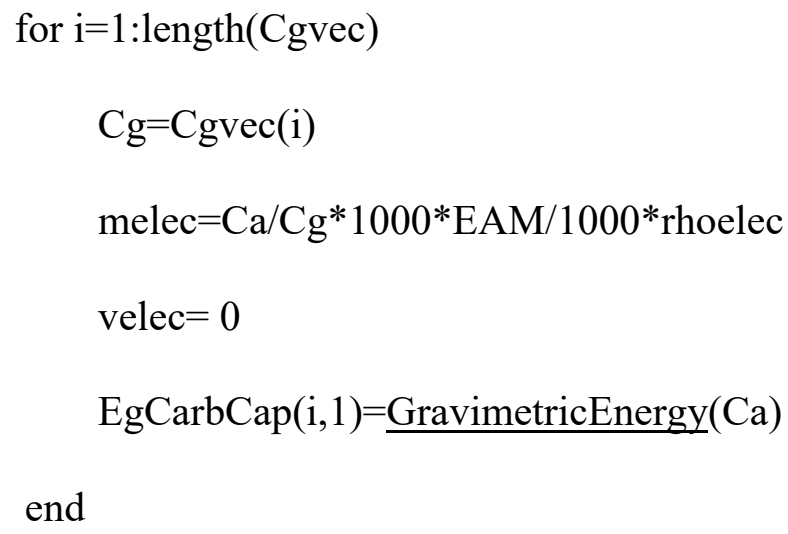


ylabel('Gravimetric energy (Wh / kg)')

legend('Liquid','Polymer')

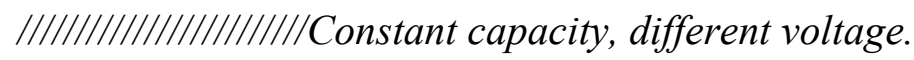

Vavevec $=(2: 0.01: 3) / /$ Varied voltage

$\mathrm{Cg}=250 / /$ Fixed capacity

$\mathrm{Ca}=1$

$\mathrm{NP}=3$

//Calculation

//Liquid

AMperc $=0.70$, Cperc $=0.20, \mathrm{EAM}=1, \mathrm{rhoB}=1.76 ;$ cathoporosity $=0.1 ; \mathrm{msep}=1.2^{*} 10^{\wedge}-$ $3 ; \mathrm{vsep}=25 * 10^{\wedge}-4 ;$ rhoelec $=1.13 / /$ Parameters for liquid

for $\mathrm{i}=1$ :length(Vavevec)

Vave $=$ Vavevec(i)

melec $=\mathrm{Ca} / \mathrm{Cg} * 1000 * \mathrm{EAM} / 1000 *$ rhoelec $/ / \mathrm{g} / \mathrm{cm} 2$

velec $=0 / / \mathrm{cm} 3 / \mathrm{cm} 2$. No extra volume, electrolyte fills the separator

EgCarbVol(i,1) $=$ GravimetricEnergy $(\mathrm{Ca}) / /$ Liquid in first column

end

\section{//Solid}

$\mathrm{AMperc}=0.5, \mathrm{Cperc}=0.20, \mathrm{rhoB}=1.2 ;$ cathoporosity $=0.2 ; \mathrm{msep}=0 ; \mathrm{vsep}=0 ;$ rhoelec $=$ rhoB; Thick= 30; eelec=0.2//Parameters for solid

for $\mathrm{i}=1$ :length(Vavevec)

Vave $=$ Vavevec $(\mathrm{i})$ 
melec $=$ Thick $/ 10000 *$ rhoelec $*(1-$ eelec $) / /$ Electrolyte amount

velec $=$ Thick $/ 10000$

EgCarbVol(i,2)=GravimetricEnergy $(\mathrm{Ca}) / /$ Polymer in second column

end

//Plots

$\underline{\operatorname{scf}}(\mathrm{FigNu})$

subplot $(1,2,2)$

plot2d(Vavevec,EgCarbVol)

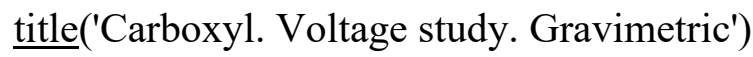

xlabel('Capacity (mAh/mg)')

ylabel('Gravimetric energy (Wh / kg)')

legend('Liquid','Polymer')

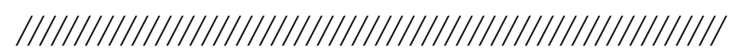

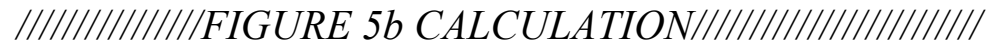

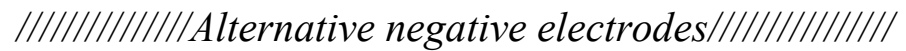

$\mathrm{Ca}=1$

NegElecvec=['Li' 'Na' 'K' 'Mg' 'Zn' 'Ca' 'Al']

CLivec $=\left[\begin{array}{llll}38861 & 1166 & 685220582013322980\end{array}\right]$

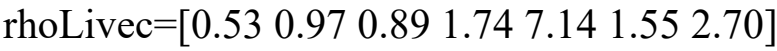

$\mathrm{NP}=3$

//PTCA-based

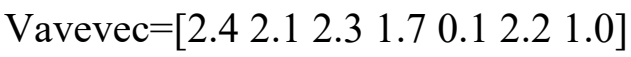


$\mathrm{Cg}=145$

$\mathrm{AMperc}=0.70, \mathrm{Cperc}=0.20, \mathrm{EAM}=1, \mathrm{rhoB}=1.76 ;$ cathoporosity $=0.1 ; \mathrm{msep}=1.2^{*} 10^{\wedge}-$ $3 ; \mathrm{vsep}=25^{*} 10^{\wedge}-4 ;$ rhoelec $=1.13$

Bperc $=1-A M p e r c-C p e r c$

//Calculation

$[\mathrm{a} b]=\operatorname{size}($ NegElecvec $)$

for $\mathrm{i}=1: \mathrm{b}$

NegElec $=$ NegElecvec(i)

$\mathrm{CLi}=$ CLivec(i)

rhoLi $=$ rhoLivec(i)

Vave $=$ Vavevec(i)

//Liquid

melec $=\mathrm{Ca} / \mathrm{Cg} * 1000 * \mathrm{EAM} / 1000 *$ rhoelec $/ / \mathrm{g} / \mathrm{cm} 2$

velec $=0 / / \mathrm{cm} 3 / \mathrm{cm} 2$. No extra volume, electrolyte fills the separator

EgLiqPTCANegEff(i,1)= GravimetricEnergy $(\mathrm{Ca})$

EvLiqPTCANegEff(i,1)=VolumetricEnergy $(\mathrm{Ca})$

end

//PAQS-based

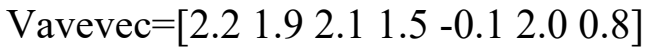

$\mathrm{Cg}=225$

$\mathrm{AMperc}=0.70$, Cperc $=0.20, \mathrm{EAM}=1, \mathrm{rhoB}=1.76 ;$ cathoporosity $=0.1 ; \mathrm{msep}=1.2^{*} 10^{\wedge} \_$ $3 ; \mathrm{vsep}=25^{*} 10^{\wedge}-4 ;$ rhoelec $=1.13 / /$ Parameters for liquid

Bperc=1-AMperc-Cperc 


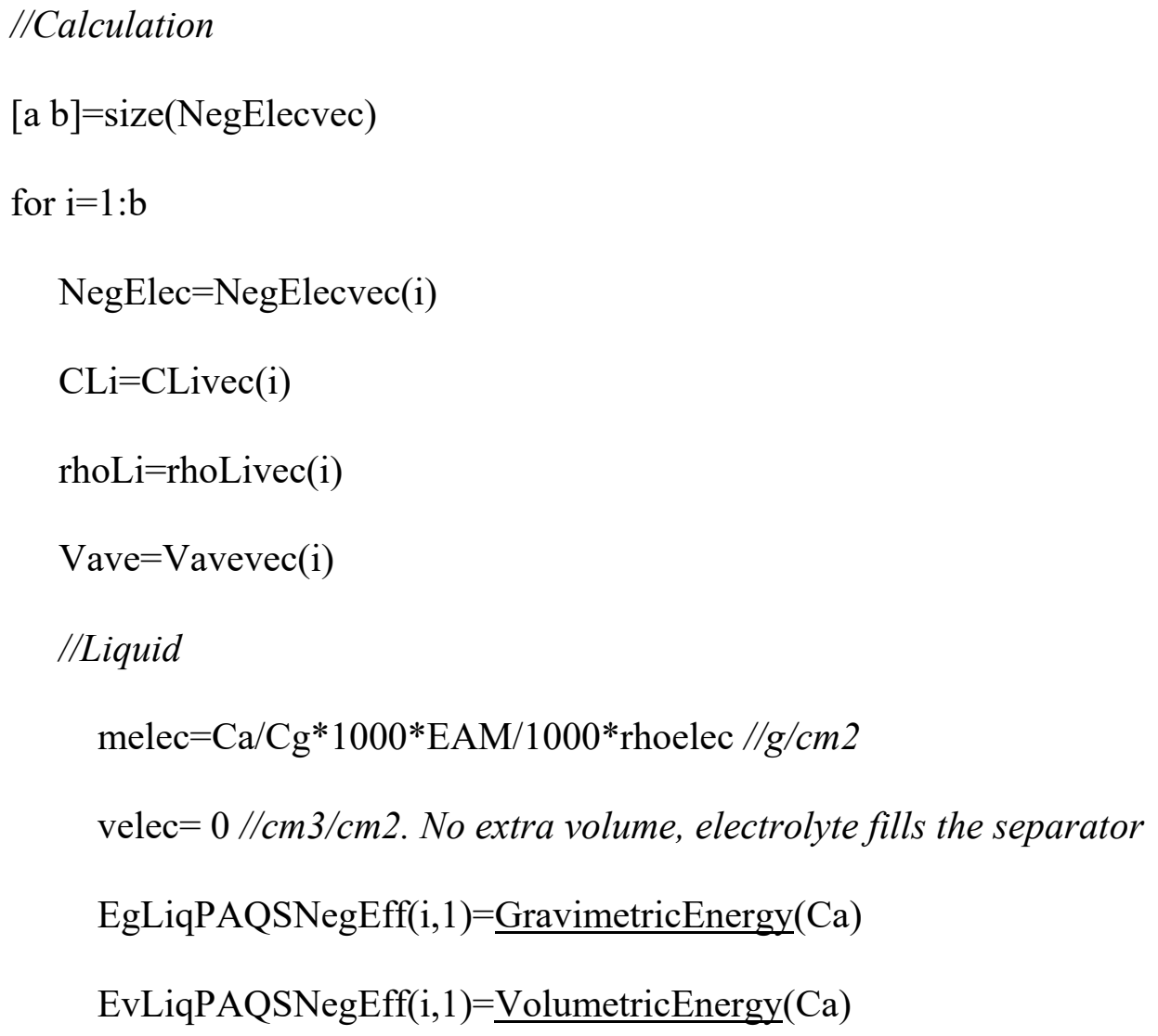

end

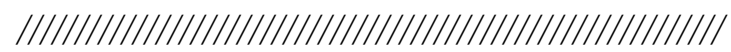

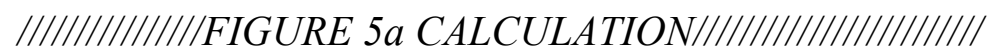

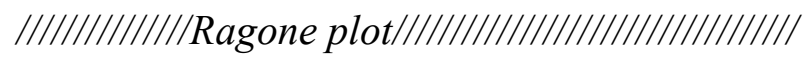

$\mathrm{EAM}=1, \mathrm{rhoB}=1.76 ;$ cathoporosity $=0.1 ; \mathrm{msep}=1.2^{*} 10^{\wedge}-3 ; \mathrm{vsep}=25^{*} 10^{\wedge}-4 ;$ rhoelec $=1.13$ $\mathrm{Ca}=1 ; \mathrm{NP}=3, \mathrm{CLi}=3861, \mathrm{rhoLi}=0.53$

$/ / L F P$

AMperc $=0.96$, Cperc $=0.02$, Bperc $=1$-AMperc-Cperc, rhoAM=3.65, Vave $=3.4$ 


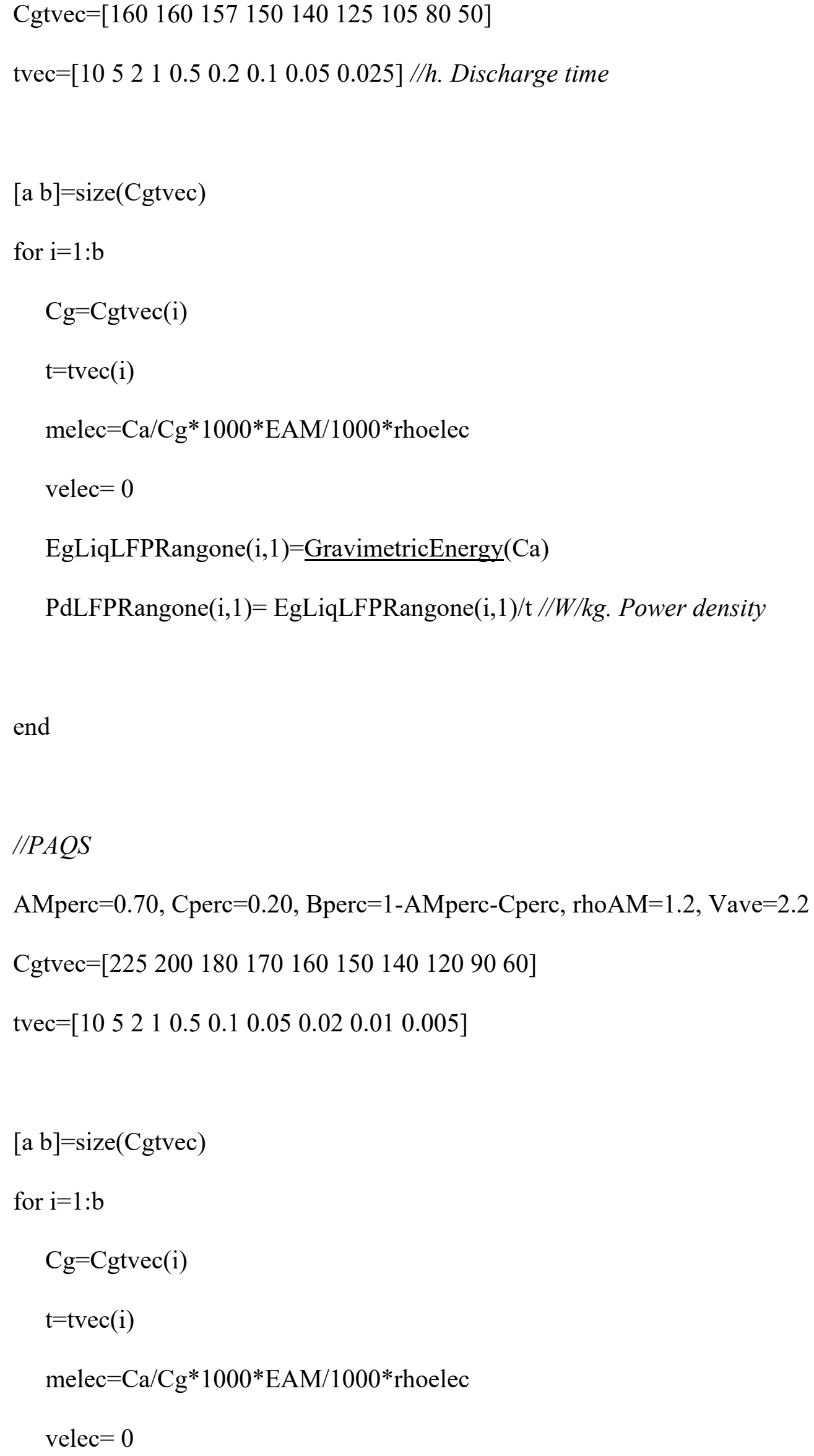


EgLiqPAQSRangone $(\mathrm{i}, 1)=\underline{\text { GravimetricEnergy }}(\mathrm{Ca})$

PdPAQSRangone $(\mathrm{i}, 1)=$ EgLiqPAQSRangone $(\mathrm{i}, 1) / \mathrm{t}$

end

$/ / S c o p$

AMperc $=0.70$, Cperc $=0.20$, Bperc $=1-$ AMperc-Cperc, rhoAM $=1.5$, Vave $=2.1$

Cgtvec $=\left[\begin{array}{lll}1285 & 1176959850750500250\end{array}\right]$

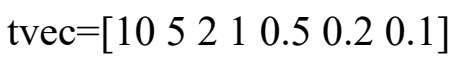

$[\mathrm{a} b]=\operatorname{size}($ Cgtvec $)$

for $i=1: b$

$\mathrm{Cg}=$ Cgtvec(i)

$\mathrm{t}=\mathrm{tvec}(\mathrm{i})$

melec $=\mathrm{Ca} / \mathrm{Cg} * 1000 * \mathrm{EAM} / 1000 *$ rhoelec

velec $=0$

EgLiqScopRangone $(\mathrm{i}, 1)=$ GravimetricEnergy $(\mathrm{Ca})$

PdScopRangone $(\mathrm{i}, 1)=$ EgLiqScopRangone $(\mathrm{i}, 1) / \mathrm{t}$

end

$/ / R a d$

AMperc $=0.70$, Cperc $=0.20$, Bperc $=1-A M p e r c-C p e r c$, rhoAM=1.2, Vave $=3.6$

Cgtvec=[110 110110110110110110988883777270 50]

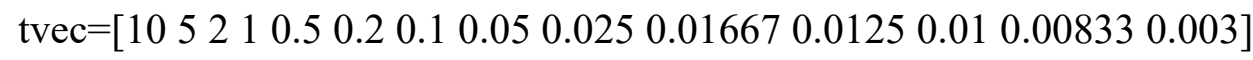


$[\mathrm{a} b]=\operatorname{size}($ Cgtvec $)$

for $i=1: b$

$\mathrm{Cg}=$ Cgtvec(i)

$\mathrm{t}=\operatorname{tvec}(\mathrm{i})$

melec $=\mathrm{Ca} / \mathrm{Cg} * 1000 * \mathrm{EAM} / 1000 *$ rhoelec

velec $=0$

EgLiqRadRangone $(\mathrm{i}, 1)=\underline{\operatorname{GravimetricEnergy}}(\mathrm{Ca})$

PdRadRangone $(\mathrm{i}, 1)=$ EgLiqRadRangone $(\mathrm{i}, 1) / \mathrm{t}$

end

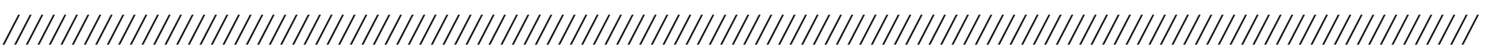

//I

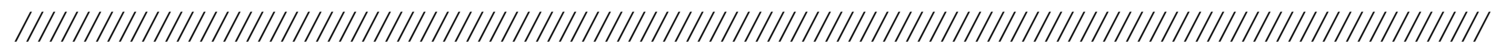

/II

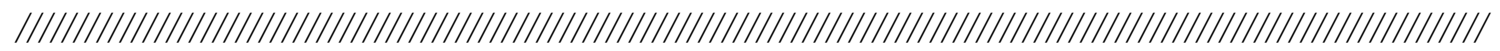

/II

$\mathrm{t}=\mathrm{toc}()$

$\operatorname{disp}\left(\mathrm{t},{ }^{\prime}\right.$ Calculation time was (s):') 


\section{References}

1. Peng, C.; Ning, G.-H.; Su, J.; Zhong, G.; Tang, W.; Tian, B.; Su, C.; Yu, D.; Zu, L.; Yang, J.; Ng, M.-F.; Hu, Y.-S.; Yang, Y.; Armand, M.; Loh, K. P., Reversible Multi-Electron Redox Chemistry of $\pi-$ conjugated N-containing Heteroaromatic Molecule-Based Organic Cathodes. Nat. Energy 2017, $2,17074$. 2. Bahceci, S.; Esat, B., A Polyacetylene Derivative with Pendant TEMPO Group as Cathode Material for Rechargeable Batteries. J. Power Sources 2013, 242, 33-40.

3. Zhu, L.; Lei, A.; Cao, Y.; Ai, X.; Yang, H., An All-organic Rechargeable Battery Using Bipolar Polyparaphenylene as a Redox-Active Cathode and Anode. Chem. Commun. 2013, 49 (6), 567-569.

4. $\quad$ Cai, Z.; Yang, G., Synthesis of Polyindole and Its Evaluation for Li-Ion Battery Applications. Synth. Met. 2010, 160 (17), 1902-1905.

5. Vlad, A.; Arnould, K.; Ernould, B.; Sieuw, L.; Rolland, J.; Gohy, J.-F., Exploring the Potential of Polymer Battery Cathodes with Electrically Conductive Molecular Backbone. J. Mater. Chem. A. 2015, 3 (21), 11189-11193.

6. $\quad$ Tang, J.; Kong, L.; Zhang, J.; Zhan, L.; Zhan, H.; Zhou, Y.; Zhan, C., Solvent-Free, Oxidatively Prepared Polythiophene: High Specific Capacity as a Cathode Active Material for Lithium Batteries. React. Funct. Polym. 2008, 68 (9), 1408-1413.

7. Aydın, M.; Esat, B.; Kılıç, Ç.; Köse, M.; Ata, A.; Yılmaz, F., A Polythiophene Derivative Bearing TEMPO as a Cathode Material for Rechargeable Batteries. Eur. Polym. J. 2011, 47 (12), 22832294.

8. Xu, F.; Chen, X.; Tang, Z.; Wu, D.; Fu, R.; Jiang, D., Redox-Active Conjugated Microporous Polymers: A New Organic Platform for Highly Efficient Energy Storage. Chem. Commun. 2014, 50 (37), 4788-4790.

9. Su, C.; Yang, F.; Ji, L.; Xu, L.; Zhang, C., Polytriphenylamine Derivative with High Free Radical Density as the Novel Organic Cathode for Lithium Ion Batteries. J. Mater. Chem. A. 2014, 2 (47), 20083-20088.

10. Sun, T.; Li, Z. J.; Wang, H. G.; Bao, D.; Meng, F. 1.; Zhang, X. B., A Biodegradable Polydopamine-Derived Electrode Material for High-Capacity and Long-Life Lithium-Ion and SodiumIon Batteries. Angew. Chem. Int. Ed. 2016, 55 (36), 10662-10666.

11. Wu, J.; Rui, X.; Wang, C.; Pei, W. B.; Lau, R.; Yan, Q.; Zhang, Q., Nanostructured Conjugated Ladder Polymers for Stable and Fast Lithium Storage Anodes with High-Capacity. Adv. Energy Mater. 2015, 5 (9), 1402189.

12. Xie, J.; Rui, X.; Gu, P.; Wu, J.; Xu, Z. J.; Yan, Q.; Zhang, Q., Novel Conjugated LadderStructured Oligomer Anode with High Lithium Storage and Long Cycling Capability. ACS Appl. Mater. Interfaces 2016, 8 (26), 16932-16938.

13. Wu, J.; Rui, X.; Long, G.; Chen, W.; Yan, Q.; Zhang, Q., Pushing Up Lithium Storage through Nanostructured Polyazaacene Analogues as Anode. Angew. Chem. Int. Ed. 2015, 54 (25), 7354-7358.

14. Zhang, S.; Huang, W.; Hu, P.; Huang, C.; Shang, C.; Zhang, C.; Yang, R.; Cui, G., Conjugated Microporous Polymers with Excellent Electrochemical Performance for Lithium and Sodium Storage. $J$. Mater. Chem. A. 2015, 3 (5), 1896-1901.

15. Song, Z.; Qian, Y.; Liu, X.; Zhang, T.; Zhu, Y.; Yu, H.; Otani, M.; Zhou, H., A Quinone-Based Oligomeric Lithium Salt for Superior Li-Organic Batteries. Energy Environ. Sci. 2014, 7 (12), 40774086.

16. Alt, H.; Binder, H.; Köhling, A.; Sandstede, G., Investigation into the Use of Quinone Compounds-for Battery Cathodes. Electrochim. Acta. 1972, 17 (5), 873-887.

17. Yokoji, T.; Kameyama, Y.; Sakaida, S.; Maruyama, N.; Satoh, M.; Matsubara, H., Steric Effects on the Cyclability of Benzoquinone-Type Organic Cathode Active Materials for Rechargeable Batteries. Chem. Lett. 2015, 44 (12), 1726-1728.

18. Yao, M.; Senoh, H.; Yamazaki, S.-i.; Siroma, Z.; Sakai, T.; Yasuda, K., High-Papacity Organic Positive-Electrode Material Based on a Benzoquinone Derivative for Use in Rechargeable Lithium Batteries. J. Power Sources. 2010, 195 (24), 8336-8340.

19. Zhang, K.; Guo, C.; Zhao, Q.; Niu, Z.; Chen, J., High-Performance Organic Lithium Batteries with an Ether-Based Electrolyte and 9,10-Anthraquinone (AQ)/CMK-3 Cathode. Adv. Sci. 2015, 2 (5), 1500018.

20. Li, H.; Duan, W.; Zhao, Q.; Cheng, F.; Liang, J.; Chen, J., 2,2'-Bis(3-hydroxy-1,4naphthoquinone)/CMK-3 Nanocomposite as Cathode Material for Lithium-Ion Batteries. Inorg. Chem. Front. 2014, 1 (2), 193-199.

21. Yokoji, T.; Matsubara, H.; Satoh, M., Rechargeable Organic Lithium-Ion Batteries Using Electron-Deficient Benzoquinones as Positive-Electrode Materials with High Discharge Voltages. J. Mater. Chem. A. 2014, 2 (45), 19347-19354. 
22. Liang, Y.; Zhang, P.; Yang, S.; Tao, Z.; Chen, J., Fused Heteroaromatic Organic Compounds for High-Power Electrodes of Rechargeable Lithium Batteries. Adv. Energy Mater. 2013, 3 (5), 600-605.

23. Iordache, A.; Maurel, V.; Mouesca, J.-M.; Pécaut, J.; Dubois, L.; Gutel, T., Monothioanthraquinone as an Organic Active Material for Greener Lithium Batteries. J. Power Sources. 2014, 267, 553-559.

24. Liang, Y.; Zhang, P.; Chen, J., Function-Oriented Design of Conjugated Carbonyl Compound Electrodes for High Energy Lithium Batteries. Chem. Sci. 2013, 4 (3), 1330-1337.

25. Wu, D.; Xie, Z.; Zhou, Z.; Shen, P.; Chen, Z., Designing High-Voltage Carbonyl-Containing Polycyclic Aromatic Hydrocarbon Cathode Materials for Li-Ion Batteries Guided by Clar's Theory. $J$. Mater. Chem. A. 2015, 3 (37), 19137-19143.

26. Han, X.; Chang, C.; Yuan, L.; Sun, T.; Sun, J., Aromatic Carbonyl Derivative Polymers as High-Performance Li-Ion Storage Materials. Adv. Mater. 2007, 19 (12), 1616-1621.

27. Vadehra, G. S.; Maloney, R. P.; Garcia-Garibay, M. A.; Dunn, B., Naphthalene Diimide Based Materials with Adjustable Redox Potentials: Evaluation for Organic Lithium-Ion Batteries. Chem. Mater. 2014, 26 (24), 7151-7157.

28. Bhosale, M. E.; Krishnamoorthy, K., Chemically Reduced Organic Small-Molecule-Based Lithium Battery with Improved Efficiency. Chem. Mater. 2015, 27 (6), 2121-2126.

29. Yao, M.; Yamazaki, S.-i.; Senoh, H.; Sakai, T.; Kiyobayashi, T., Crystalline Polycyclic Quinone Derivatives as Organic Positive-Electrode Materials for Use in Rechargeable Lithium Batteries. Mater. Sci. Eng. B 2012, 177 (6), 483-487.

30. Zou, Q.; Wang, W.; Wang, A.; Yu, Z.; Yuan, K., Preparation of the Tetrahydro-Hexaquinone as a Novel Cathode Material for Rechargeable Lithium Batteries. Mater. Lett. 2014, 117, 290-293.

31. Lee, J.; Park, M. J., Tattooing Dye as a Green Electrode Material for Lithium Batteries. Adv. Energy Mater. 2017, 7 (12), 1602279.

32. Lee, J.; Kim, H.; Park, M. J., Long-Life, High-Rate Lithium-Organic Batteries Based on Naphthoquinone Derivatives. Chem. Mater. 2016, 28 (7), 2408-2416.

33. Luo, Z.; Liu, L.; Zhao, Q.; Li, F.; Chen, J., An Insoluble Benzoquinone-Based Organic Cathode for Use in Rechargeable Lithium-Ion Batteries. Angew. Chem. Int. Ed. 2017, 129 (41), 12735-12739.

34. Kim, H.; Seo, D.-H.; Yoon, G.; Goddard, W. A.; Lee, Y. S.; Yoon, W.-S.; Kang, K., The Reaction Mechanism and Capacity Degradation Model in Lithium Insertion Organic Cathodes, $\mathrm{Li}_{2} \mathrm{C}_{6} \mathrm{O}_{6}$, Using Combined Experimental and First Principle Studies. J. Phys. Chem. Lett. 2014, 5 (17), 3086-3092.

35. Xiang, J.; Chang, C.; Li, M.; Wu, S.; Yuan, L.; Sun, J., A Novel Coordination Polymer as Positive Electrode Material for Lithium Ion Battery. Cryst. Growth Des. 2008, 8 (1), 280-282.

36. Chen, H.; Armand, M.; Demailly, G.; Dolhem, F.; Poizot, P.; Tarascon, J. M., From Biomass to a Renewable $\mathrm{Li}_{\mathrm{X}} \mathrm{C}_{6} \mathrm{O}_{6}$ Organic Electrode for Sustainable Li-Ion Batteries. ChemSusChem 2008, 1 (4), $348-355$.

37. Luo, C.; Huang, R.; Kevorkyants, R.; Pavanello, M.; He, H.; Wang, C., Self-Assembled Organic Nanowires for High Power Density Lithium Ion Batteries. Nano Lett. 2014, 14 (3), 1596-1602.

38. Gottis, S.; Barrès, A.-L.; Dolhem, F.; Poizot, P., Voltage Gain in Lithiated Enolate-Based Organic Cathode Materials by Isomeric Effect. ACS Appl. Mater. Interfaces 2014, 6 (14), 10870-10876.

39. Shimizu, A.; Kuramoto, H.; Tsujii, Y.; Nokami, T.; Inatomi, Y.; Hojo, N.; Suzuki, H.; Yoshida, J.-i., Introduction of Two Lithiooxycarbonyl Groups Enhances Cyclability of Lithium Batteries with Organic Cathode Materials. J. Power Sources 2014, 260, 211-217.

40. Zeng, R.-H.; Li, X.-P.; Qiu, Y.-C.; Li, W.-S.; Yi, J.; Lu, D.-S.; Tan, C.-L.; Xu, M.-Q., Synthesis and Properties of a Lithium-Organic Coordination Compound as Lithium-Inserted Material for Lithium Ion Batteries. Electrochem. Commun. 2010, 12 (9), 1253-1256.

41. Wan, W.; Lee, H.; Yu, X.; Wang, C.; Nam, K.-W.; Yang, X.-Q.; Zhou, H., Tuning the Electrochemical Performances of Anthraquinone Organic Cathode Materials for Li-Ion Batteries through the Sulfonic Sodium Functional Group. RSC Adv. 2014, 4 (38), 19878-19882.

42. Renault, S.; Geng, J.; Dolhem, F.; Poizot, P., Evaluation of Polyketones with $N$-cyclic Structure as Electrode Material for Electrochemical Energy Storage: Case of Pyromellitic Diimide Dilithium Salt. Chem. Commun. 2011, 47 (8), 2414-2416.

43. Luo, C.; Ji, X.; Hou, S.; Eidson, N.; Fan, X.; Liang, Y.; Deng, T.; Jiang, J.; Wang, C., Azo Compounds Derived from Electrochemical Reduction of Nitro Compounds for High Performance Li-Ion Batteries. Adv. Mater. 2018, 30 (23), 1706498.

44. Armand, M.; Grugeon, S.; Vezin, H.; Laruelle, S.; Ribière, P.; Poizot, P.; Tarascon, J. M., Conjugated dicarboxylate Anodes for Li-Ion Batteries. Nat. Mater. 2009, 8, 120-125.

45. Wang, L.; Mou, C.; Wu, B.; Xue, J.; Li, J., Alkaline Earth Metal Terephthalates $\mathrm{MC}_{8} \mathrm{H}_{4} \mathrm{O}_{4}(\mathrm{M}=$ $\mathrm{Ca}, \mathrm{Sr}, \mathrm{Ba})$ as Anodes for Lithium Ion Batteries. Electrochim. Acta 2016, 196, 118-124. 
46. Xue, J.; Fan, C.; Deng, Q.; Zhao, M.; Wang, L.; Zhou, A.; Li, J., Silver Terephthalate $\left(\mathrm{Ag}_{2} \mathrm{C}_{8} \mathrm{H}_{4} \mathrm{O}_{4}\right)$ Offering in-situ Formed Metal/Organic Nanocomposite as the Highly Efficient Organic Anode in Li-Ion and Na-Ion Batteries. Electrochim. Acta 2016, 219, 418-424.

47. Lee, H. H.; Park, Y.; Shin, K.-H.; Lee, K. T.; Hong, S. Y., Abnormal Excess Capacity of Conjugated Dicarboxylates in Lithium-Ion Batteries. ACS Appl. Mater. Interfaces 2014, 6 (21), 1911819126.

48. Fédèle, L.; Sauvage, F.; Bécuwe, M., Hyper-Conjugated Lithium Carboxylate Based on a Perylene Unit for High-Rate Organic Lithium-Ion Batteries. J. Mater. Chem. A. 2014, 2 (43), 1822518228.

49. Song, Z.; Zhan, H.; Zhou, Y., Anthraquinone Based Polymer as High Performance Cathode Material for Rechargeable Lithium Batteries. Chem. Commun. 2009, 0, 448-450.

50. Song, Z.; Xu, T.; Gordin, M. L.; Jiang, Y.-B.; Bae, I.-T.; Xiao, Q.; Zhan, H.; Liu, J.; Wang, D., Polymer-Graphene Nanocomposites as Ultrafast-Charge and -Discharge Cathodes for Rechargeable Lithium Batteries. Nano Lett. 2012, 12 (5), 2205-2211.

51. Xu, W.; Read, A.; Koech, P. K.; Hu, D.; Wang, C.; Xiao, J.; Padmaperuma, A. B.; Graff, G. L.; Liu, J.; Zhang, J.-G., Factors Affecting the Battery Performance of Anthraquinone-Based Organic Cathode Materials. J. Mater. Chem. 2012, 22 (9), 4032-4039.

52. Song, Z.; Qian, Y.; Gordin, M. L.; Tang, D.; Xu, T.; Otani, M.; Zhan, H.; Zhou, H.; Wang, D., Polyanthraquinone as a Reliable Organic Electrode for Stable and Fast Lithium Storage. Angew. Chem. Int. Ed. 2015, 54 (47), 13947-13951.

53. Song, Z.; Qian, Y.; Zhang, T.; Otani, M.; Zhou, H., Poly(benzoquinonyl sulfide) as a HighEnergy Organic Cathode for Rechargeable Li and Na Batteries. Adv. Sci. 2015, 2 (9), 1500124.

54. Bitenc, J.; Pirnat, K.; Mali, G.; Novosel, B.; Randon Vitanova, A.; Dominko, R., Poly(hydroquinoyl-benzoquinonyl sulfide) as an Active Material in $\mathrm{Mg}$ and Li Organic Batteries. Electrochem. Commun. 2016, 69, 1-5.

55. Liu, K.; Zheng, J.; Zhong, G.; Yang, Y., Poly (2,5-dihydroxy-1,4-benzoquinonyl sulfide) (PDBS) as a Cathode Material for Lithium Ion Batteries. J. Mater. Chem. 2011, 21 (12), 4125-4131.

56. Le Gall, T.; Reiman, K. H.; Grossel, M. C.; Owen, J. R., Poly (2,5-dihydroxy-1,4-benzoquinone3,6-methylene): A New Organic Polymer as Positive Electrode Material for Rechargeable Lithium Batteries. J. Power Sources 2003, 119, 316-320.

57. Oyama, N.; Sarukawa, T.; Mochizuki, Y.; Shimomura, T.; Yamaguchi, S., Significant Effects of Poly(3,4-ethylenedioxythiophene) Additive on Redox Responses of Poly(2,5-dihydroxy-1,4benzoquinone-3,6-methylene) Cathode for Rechargeable Li Batteries. J. Power Sources 2009, 189 (1), 230-239.

58. Kassam, A.; Burnell, D. J.; Dahn, J., Lithiated 1, 4, 5, 8-naphthalenetetraol Formaldehyde Polymer, an Organic Cathode Material. Electrochem. Solid State Lett. 2011, 14 (2), A22-A23.

59. Zhao, L.; Wang, W.; Wang, A.; Yuan, K.; Chen, S.; Yang, Y., A Novel Polyquinone Cathode Material for Rechargeable Lithium Batteries. J. Power Sources 2013, 233, 23-27.

60. Häupler, B.; Hagemann, T.; Friebe, C.; Wild, A.; Schubert, U. S., Dithiophenedione-Containing Polymers for Battery Application. ACS Appl. Mater. Interfaces 2015, 7 (6), 3473-3479.

61. Liu, T.; Kim, K. C.; Lee, B.; Chen, Z.; Noda, S.; Jang, S. S.; Lee, S. W., Self-Polymerized Dopamine as an Organic Cathode for Li-and Na-Ion Batteries. Energy Environ. Sci. 2017, 10 (1), 205215.

62. Oyaizu, K.; Choi, W.; Nishide, H., Functionalization of Poly(4-chloromethylstyrene) with Anthraquinone Pendants for Organic Anode-Active Materials. Polym. Adv. Technol. 2011, 22 (8), 12421247.

63. Nokami, T.; Matsuo, T.; Inatomi, Y.; Hojo, N.; Tsukagoshi, T.; Yoshizawa, H.; Shimizu, A.; Kuramoto, H.; Komae, K.; Tsuyama, H.; Yoshida, J.-i., Polymer-Bound Pyrene-4,5,9,10-tetraone for Fast-Charge and-Discharge Lithium-Ion Batteries with High Capacity. J. Am. Chem. Soc. 2012, 134 (48), 19694-19700.

64. Xiang, J.; Chang, C.; Li, M.; Wu, S.; Yuan, L.; Sun, J., A Novel Coordination Polymer as Positive Electrode Material for Lithium Ion Battery. Cryst. Growth Des. 2007, 8 (1), 280-282.

65. Oyaizu, K.; Hatemata, A.; Choi, W.; Nishide, H., Redox-Active Polyimide/Carbon Nanocomposite Electrodes for Reversible Charge Storage at Negative Potentials: Expanding the Functional Horizon of Polyimides. J. Mater. Chem. 2010, 20 (26), 5404-5410.

66. Song, Z.; Zhan, H.; Zhou, Y., Polyimides: Promising Energy-Storage Materials. Angew. Chem. Int. Ed. 2010, 49 (45), 8444-8448.

67. Wu, H.; Shevlin, S. A.; Meng, Q.; Guo, W.; Meng, Y.; Lu, K.; Wei, Z.; Guo, Z., Flexible and Binder-Free Organic Cathode for High-Performance Lithium-Ion Batteries. Adv. Mater. 2014, 26 (20), 3338-3343. 
68. Wu, H.; Meng, Q.; Yang, Q.; Zhang, M.; Lu, K.; Wei, Z., Large-Area Polyimide/SWCNT Nanocable Cathode for Flexible Lithium-Ion Batteries. Adv. Mater. 2015, 27 (41), 6504-6510.

69. Hernández, G.; Casado, N.; Coste, R.; Shanmukaraj, D.; Rubatat, L.; Armand, M.; Mecerreyes, D., Redox-Active Polyimide-Polyether Block Copolymers as Electrode Materials for Lithium Batteries. RSC Adv. 2015, 5 (22), 17096-17103.

70. Schon, T. B.; Tilley, A. J.; Bridges, C. R.; Miltenburg, M. B.; Seferos, D. S., Bio-Derived Polymers for Sustainable Lithium-Ion Batteries. Adv. Funct. Mater. 2016, 26 (38), 6896-6903.

71. Wu, H.; Wang, K.; Meng, Y.; Lu, K.; Wei, Z., An Organic Cathode Material Based On a Polyimide/CNT Nanocomposite for Lithium Ion Batteries. J. Mater. Chem. A. 2013, 1 (21), 6366-6372.

72. Sharma, P.; Damien, D.; Nagarajan, K.; Shaijumon, M. M.; Hariharan, M., Perylene-PolyimideBased Organic Electrode Materials for Rechargeable Lithium Batteries. J. Phys. Chem. Lett. 2013, 4 (19), 3192-3197.

73. Tian, D.; Zhang, H.-Z.; Zhang, D.-S.; Chang, Z.; Han, J.; Gao, X.-P.; Bu, X.-H., Li-Ion Storage and Gas Adsorption Properties of Porous Polyimides (PIs). RSC Adv. 2014, 4 (15), 7506-7510.

74. Geng, J.; Bonnet, J.-P.; Renault, S.; Dolhem, F.; Poizot, P., Evaluation of Polyketones with Ncyclic Structure as Electrode Material for Electrochemical Energy Storage: Case of Tetraketopiperazine Unit. Energy Environ. Sci. 2010, 3 (12), 1929-1933.

75. Schmidt, D.; Häupler, B.; Stolze, C.; Hager, M. D.; Schubert, U. S., Poly [N-(10-oxo-2vinylanthracen-9 (10H)-ylidene) Cyanamide] as a Novel Cathode Material for Li-Organic Batteries. $J$. Polym. Sci. Polym. Chem. 2015, 53 (21), 2517-2523.

76. Patil, N.; Aqil, A.; Ouhib, F.; Admassie, S.; Inganäs, O.; J é rôme, C.; Detrembleur, C., Bioinspired Redox-Active Catechol-Bearing Polymers as Ultrarobust Organic Cathodes for Lithium Storage. Adv. Mater. 2017, 29 (40), 1703373.

77. $\quad$ Liang, Y.; Chen, Z.; Jing, Y.; Rong, Y.; Facchetti, A.; Yao, Y., Heavily n-dopable $\pi$-conjugated Redox Polymers with Ultrafast Energy Storage Capability. J. Am. Chem. Soc. 2015, 137 (15), 4956-4959.

78. Visco, S. J.; DeJonghe, L. C., Ionic Conductivity of Organosulfur Melts for Advanced Storage Electrodes. J. Electrochem. Soc. 1988, 135 (12), 2905-2909.

79. Genieès, E.; Picart, S., Is the Use of Polyaniline Associated with Sulfur Compounds of Interest for Battery Electrodes? Synth. Met. 1995, 69 (1-3), 165-166.

80. Wu, M.; Cui, Y.; Bhargav, A.; Losovyj, Y.; Siegel, A.; Agarwal, M.; Ma, Y.; Fu, Y., Organotrisulfide: A High Capacity Cathode Material for Rechargeable Lithium Batteries. Angew. Chem. Int. Ed. 2016, 55 (34), 10027-10031.

81. Wu, M.; Bhargav, A.; Cui, Y.; Siegel, A.; Agarwal, M.; Ma, Y.; Fu, Y., Highly Reversible Diphenyl Trisulfide Catholyte for Rechargeable Lithium Batteries. ACS Energy Lett. 2016, 1 (6), 12211226.

82. Shimizu, T.; Wang, H.; Tanifuji, N.; Matsumura, D.; Yoshimura, M.; Nakanishi, K.; Ohta, T.; Yoshikawa, H., Rechargeable Batteries Based on Stable Redox Reactions of Disulfide Included in a Metal-Organic Framework as Ligands. Chem. Lett. 2018, 47 (5), 678-681.

83. Guo, W.; Bhargav, A.; Ackerson, J. D.; Cui, Y.; Ma, Y.; Fu, Y., Mixture Is Better: Enhanced Electrochemical Performance of Phenyl Selenosulfide in Rechargeable Lithium Batteries. Chem. Commun. 2018, 54 (64), 8873-8876.

84. Bhargav, A.; Patil, S. V.; Fu, Y., A Phenyl Disulfide@CNT Composite Cathode for Rechargeable Lithium Batteries. Sustainable Energy Fuels 2017, 1 (5), 1007-1012.

85. Guo, W.; Wawrzyniakowski, Z. D.; Cerda, M. M.; Bhargav, A.; Pluth, M. D.; Ma, Y.; Fu, Y., Bis (aryl) Tetrasulfides as Cathode Materials for Rechargeable Lithium Batteries. Chem. Eur. J. 2017, 23 (67), 16941-16947.

86. Bhargav, A.; Ma, Y.; Shashikala, K.; Cui, Y.; Losovyj, Y.; Fu, Y., The Unique Chemistry of Thiuram Polysulfides Enables Energy Dense Lithium Batteries. J. Mater. Chem. A. 2017, 5 (47), 2500525013.

87. Davoglio, R. A.; Biaggio, S. R.; Rocha-Filho, R. C.; Bocchi, N., Bilayered Nanofilm of Polypyrrole and Poly (DMcT) for High-Performance Battery Cathodes. J. Power Sources 2010, 195 (9), 2924-2927.

88. Jin, L.; Wang, G.; Li, X.; Li, L., Poly (2, 5-dimercapto-1, 3, 4-thiadiazole)/Sulfonated Graphene Composite as Cathode Material for Rechargeable Lithium Batteries. J. Appl. Electrochem. 2011, 41 (4), $377-382$.

89. Kiya, Y.; Iwata, A.; Sarukawa, T.; Henderson, J. C.; Abruña, H. D., Poly [dithio-2, 5-(1, 3, 4thiadiazole)] (PDMcT)-poly (3, 4-ethylenedioxythiophene) (PEDOT) Composite Cathode for HighEnergy Lithium/Lithium-Ion Rechargeable Batteries. J. Power Sources 2007, 173 (1), 522-530. 
90. Deng, S.-R.; Kong, L.-B.; Hu, G.-Q.; Wu, T.; Li, D.; Zhou, Y.-H.; Li, Z.-Y., Benzene-Based Polyorganodisulfide Cathode Materials for Secondary Lithium Batteries. Electrochim. Acta 2006, 51 (13), 2589-2593.

91. Su, Y.-Z.; Dong, W.; Zhang, J.-H.; Song, J.-H.; Zhang, Y.-H.; Gong, K.-C., Poly [bis (2aminophenyloxy) disulfide]: A Polyaniline Derivative Containing Disulfide Bonds as a Cathode Material for Lithium battery. Polymer 2007, 48 (1), 165-173.

92. Wang, G.; Yang, X.; Sun, Y.; Bao, H.; Li, X., Aniline-Based Disulfide/Aniline Copolymers as a High Energy-Storage Material. Macromol. Chem. Phys. 2009, 210 (24), 2118-2124.

93. Li, Y.; Zhan, H.; Kong, L.; Zhan, C.; Zhou, Y., Electrochemical Properties of PABTH as Cathode Materials for Rechargeable Lithium Battery. Electrochem. Commun. 2007, 9 (5), 1217-1221.

94. Li, Y.; Wu, L.; Zhan, H.; Song, Z.; Kong, L.; Li, Z.; Zhou, Y., Promising Solvent of 12, 12diethyl-2, 5, 8-trioxa-12-silatetradecane for Lithium Secondary Battery. Electrochem. Commun. 2008, 10 (5), 753-756.

95. Zhang, S.-c.; Zhang, L.; Wang, W.-k.; Xue, W.-j., A Novel Cathode Material Based on Polyaniline Used for Lithium/Sulfur Secondary Battery. Synth. Met. 2010, 160 (17-18), 2041-2044.

96. Trofimov, B.; Myachina, G.; Rodionova, I.; Mal'kina, A.; Dorofeev, I.; Vakul'skaya, T.; Sinegovskaya, L.; Skotheim, T., Ethynedithiol-Based Polyeneoligosulfides as Active Cathode Materials for Lithium-Sulfur Batteries. J. Appl. Polym. Sci. 2008, 107 (2), 784-787.

97. Zhou, J.; Qian, T.; Xu, N.; Wang, M.; Ni, X.; Liu, X.; Shen, X.; Yan, C., Selenium-Doped Cathodes for Lithium-Organosulfur Batteries with Greatly Improved Volumetric Capacity and Coulombic Efficiency. Adv. Mater. 2017, 29 (33), 1701294.

98. Kim, H.; Lee, J.; Ahn, H.; Kim, O.; Park, M. J., Synthesis of Three-Dimensionally Interconnected Sulfur-Rich Polymers for Cathode Materials of High-rate Lithium-Sulfur Batteries. Nat. Commun. 2015, 6, 7278 .

99. Zeng, S.; Li, L.; Xie, L.; Zhao, D.; Zhou, N.; Wang, N.; Chen, S., Graphene-Supported Highly Crosslinked Organosulfur Nanoparticles as Cathode Materials for High-Rate, Long-Life Lithium-Sulfur Battery. Carbon 2017, 122, 106-113.

100. Chung, W. J.; Griebel, J. J.; Kim, E. T.; Yoon, H.; Simmonds, A. G.; Ji, H. J.; Dirlam, P. T.; Glass, R. S.; Wie, J. J.; Nguyen, N. A.; Guralnick, B. W.; Park, J.; Somogyi, Á.; Theato, P.; Mackay, M. E.; Sung, Y.-E.; Char, K.; Pyun, J., The Use of Elemental Sulfur as an Alternative Feedstock for Polymeric Materials. Nat. Chem. 2013, 5, 518.

101. Hu, G.; Sun, Z.; Shi, C.; Fang, R.; Chen, J.; Hou, P.; Liu, C.; Cheng, H. M.; Li, F., A SulfurRich Copolymer@CNT Hybrid Cathode with Dual-Confinement of Polysulfides for High-Performance Lithium-Sulfur Batteries. Adv. Mater. 2017, 29 (11), 1603835.

102. Ghosh, A.; Shukla, S.; Khosla, G. S.; Lochab, B.; Mitra, S., Sustainable Sulfur-Rich Copolymer/Graphene Composite as Lithium-Sulfur Battery Cathode with Excellent Electrochemical Performance. Sci. Rep. 2016, 6, 25207.

103. Zeng, S.; Li, L.; Yu, J.; Wang, N.; Chen, S., Highly Crosslinked Organosulfur Copolymer Nanosheets with Abundant Mesopores as Cathode Materials for Efficient Lithium-Sulfur Batteries. Electrochim. Acta 2018, 263, 53-59.

104. Gomez, I.; Mecerreyes, D.; Blazquez, J. A.; Leonet, O.; Youcef, H. B.; Li, C.; Gómez-Cámer, J. L.; Bondarchuk, O.; Rodriguez-Martinez, L., Inverse Vulcanization of Sulfur with Divinylbenzene: Stable and Easy Processable Cathode Material for Lithium-Sulfur Batteries. J. Power Sources 2016, 329, 72-78. 105. Zhang, Y.; Griebel, J. J.; Dirlam, P. T.; Nguyen, N. A.; Glass, R. S.; Mackay, M. E.; Char, K.; Pyun, J., Inverse Vulcanization of Elemental Sulfur and Styrene for Polymeric Cathodes in Li-S Batteries. J. Polym. Sci. Polym. Chem. 2017, 55 (1), 107-116.

106. Zhang, J. Y.; Kong, L. B.; Zhan, L. Z.; Tang, J.; Zhan, H.; Zhou, Y. H.; Zhan, C. M., Sulfides Organic Polymer: Novel Cathode Active Material for Rechargeable Lithium Batteries. J. Power Sources 2007, 168 (1), 278-281.

107. Zhang, J.; Kong, L.; Zhan, L.; Tang, J.; Zhan, H.; Zhou, Y.; Zhan, C., Aliphatic Thioether Polymers as Novel Cathode Active Materials for Rechargeable Lithium Battery. Electrochem. Commun. 2008, 10 (10), 1551-1554.

108. Zhang, J.; Song, Z.; Zhan, L.; Tang, J.; Zhan, H.; Zhou, Y.; Zhan, C., Poly (ethene-1, 1, 2, 2tetrathiol): Novel Cathode Material with High Specific Capacity for Rechargeable Lithium Batteries. $J$. Power Sources 2009, 186 (2), 496-499.

109. Zhan, L.; Song, Z.; Shan, N.; Zhang, J.; Tang, J.; Zhan, H.; Zhou, Y.; Li, Z.; Zhan, C., Poly (tetrahydrobenzodithiophene): High Discharge Specific Capacity as Cathode Material for Lithium Batteries. J. Power Sources 2009, 193 (2), 859-863. 
110. Zhan, L. Z.; Song, Z. P.; Zhang, J. Y.; Tang, J.; Zhan, H.; Zhou, Y. H.; Zhan, C. M., Synthesis and Properties of Novel Organic Thiolane Polymer as Cathode Material for Rechargeable Lithium Batteries. J. Appl. Electrochem. 2008, 38 (12), 1691-1694.

111. Nakahara, K.; Iriyama, J.; Iwasa, S.; Suguro, M.; Satoh, M.; Cairns, E. J., Cell Properties for Modified PTMA Aathodes of Organic Radical Batteries. J. Power Sources 2007, 165 (1), 398-402.

112. Nakahara, K.; Iriyama, J.; Iwasa, S.; Suguro, M.; Satoh, M.; Cairns, E. J., Al-Laminated Film Packaged Organic Radical Battery for High-Power Applications. J. Power Sources 2007, 163 (2), 1110 1113.

113. Kim, J.-K.; Scheers, J.; Ahn, J.-H.; Johansson, P.; Matic, A.; Jacobsson, P., Nano-Fibrous Polymer Films for Organic Rechargeable Batteries. J. Mater. Chem. A. 2013, 1 (7), 2426-2430.

114. Kim, J.-K., Micro-Fibrous Organic Radical Electrode to Improve the Electrochemical Properties of Organic Rechargeable Batteries. J. Power Sources 2013, 242, 683-686.

115. Liu, C.; Chen, J.; Wang, F.; Yi, B., Improvement of Electrochemical Properties of PTMA Cathode by Using Carbon Blacks with High Specific Surface Area. Russ. J. Electrochem. 2012, 48 (11), $1052-1057$.

116. Bugnon, L.; Morton, C. J.; Novak, P.; Vetter, J.; Nesvadba, P., Synthesis of Poly (4methacryloyloxy-TEMPO) via Group-Ttransfer Polymerization and Its Evaluation in Organic Radical Battery. Chem. Mater. 2007, 19 (11), 2910-2914.

117. Iwasa, S.; Nishi, T.; Nakamura, S., Enhancement of Rapid Charging Capability of Organic Radical Battery Using Ethylene Carbonate-Based Electrolyte Containing LiFSI. J. Power Sources 2018, 402, 157-162.

118. Cheng, Y.-Y.; Li, C.-C.; Lee, J.-T., Electrochemical Behavior of Organic Radical Polymer Cathodes in Organic Radical Batteries with $N$-butyl- $N$-methylpyrrolidinium Bis(trifluoromethylsulfonyl)imide Ionic Liquid Electrolytes. Electrochim. Acta 2012, 66, 332-339.

119. Wang, S.; Li, F.; Easley, A. D.; Lutkenhaus, J. L., Real-Time Insight into the Doping Mechanism of Redox-Active Organic Radical Polymers. Nat. Mater. 2019, 18 (1), 69-75.

120. Gerlach, P.; Burges, R.; Lex-Balducci, A.; Schubert, U. S.; Balducci, A., The Influence of the Electrolyte Composition on the Electrochemical Behaviour of Cathodic Materials for Organic Radical Batteries. J. Power Sources 2018, 405, 142-149.

121. Vereshchagin, A. A.; Vlasov, P. S.; Konev, A. S.; Yang, P.; Grechishnikova, G. A.; Levin, O. V., Novel Highly Conductive Cathode Material Based on Stable-Radical Organic Framework and Polymerized Nickel Complex for Electrochemical Energy Storage Devices. Electrochim. Acta 2019, 295, $1075-1084$.

122. Suga, T.; Konishi, H.; Nishide, H., Photocrosslinked Nitroxide Polymer Cathode-Active Materials for Application in an Organic-Based Paper Battery. Chem. Commun. 2007, 0, 1730-1732.

123. Koshika, K.; Sano, N.; Oyaizu, K.; Nishide, H., An Ultrafast Chargeable Polymer Electrode Based on the Combination of Nitroxide Radical and Aqueous Electrolyte. Chem. Commun. 2009, 0, 836838.

124. Sukegawa, T.; Sato, K.; Oyaizu, K.; Nishide, H., Efficient Charge Transport of a Radical Polyether/SWCNT Composite Electrode for an Organic Radical Battery with High Charge-Storage Density. RSC Adv. 2015, 5 (20), 15448-15452.

125. Schwartz, P.-O.; Pejic, M.; Wachtler, M.; Bäuerle, P., Synthesis and Characterization of Electroactive PEDOT-TEMPO Polymers as Potential Cathode Materials in Rechargeable Batteries. Synth. Met. 2018, 243, 51-57.

126. Aqil, M.; Ouhib, F.; Aqil, A.; El Idrissi, A.; Detrembleur, C.; Jérôme, C., Polymer Ionic Liquid Bearing Radicals as an Active Material for Organic Batteries with Ultrafast Charge-Discharge Rate. Eur. Polym. J. 2018, 106, 242-248.

127. Tian, B.; Ding, Z.; Ning, G.-H.; Tang, W.; Peng, C.; Liu, B.; Su, J.; Su, C.; Loh, K. P., Amino Group Enhanced Phenazine Derivatives as Electrode Materials for Lithium Storage. Chem. Commun. 2017, 53 (20), 2914-2917.

128. Hanyu, Y.; Sugimoto, T.; Ganbe, Y.; Masuda, A.; Honma, I., Multielectron Redox Compounds for Organic Cathode Quasi-Solid State Lithium Battery. J. Electrochem. Soc. 2014, 161 (1), A6-A9.

129. Yao, M.; Senoh, H.; Sakai, T.; Kiyobayashi, T., Redox Active Poly (N-vinylcarbazole) for Use in Rechargeable Lithium Batteries. J. Power Sources 2012, 202, 364-368.

130. Häupler, B.; Burges, R.; Friebe, C.; Janoschka, T.; Schmidt, D.; Wild, A.; Schubert, U. S., Poly (exTTF): A Novel Redox-Active Polymer as Active Material for Li-Organic Batteries. Macromol. Rapid Commun. 2014, 35 (15), 1367-1371.

131. Häupler, B.; Burges, R.; Janoschka, T.; Jähnert, T.; Wild, A.; Schubert, U. S., PolyTCAQ in Organic Batteries: Enhanced Capacity at Constant Cell Potential Using Two-Electron-Redox-Reactions. J. Mater. Chem. A. 2014, 2 (24), 8999-9001. 
132. Feng, J.; Cao, Y.; Ai, X.; Yang, H., Polytriphenylamine: A High Power and High Capacity Cathode Material for Rechargeable Lithium Batteries. J. Power Sources 2008, 177 (1), 199-204.

133. Sano, N.; Tomita, W.; Hara, S.; Min, C.-M.; Lee, J.-S.; Oyaizu, K.; Nishide, H., Polyviologen Hydrogel with High-Rate Capability for Anodes Toward an Aqueous Electrolyte-Type and OrganicBased Rechargeable Device. ACS Appl. Mater. Interfaces 2013, 5 (4), 1355-1361.

134. Su, C.; Yang, F.; Ye, Y.; Xu, L.; Wang, L.; Zhang, C., Poly [tris(thienylphenyl)amine] Derivatives as a Performance-Improved Cathode Material for Lithium Ion Batteries. J. Electrochem. Soc. 2013, 160 (11), A2021-A2026.

135. Speer, M. E.; Kolek, M.; Jassoy, J. J.; Heine, J.; Winter, M.; Bieker, P. M.; Esser, B., Thianthrene-Functionalized Polynorbornenes as High-Voltage Materials for Organic Cathode-Based Dual-Ion batteries. Chem. Commun. 2015, 51 (83), 15261-15264.

136. Kolek, M.; Otteny, F.; Schmidt, P.; Mück-Lichtenfeld, C.; Einholz, C.; Becking, J.; Schleicher, E.; Winter, M.; Bieker, P.; Esser, B., Ultra-High Cycling Stability of Poly(vinylphenothiazine) as a Battery Cathode Material Resulting From $\pi-\pi$ Interactions. Energy Environ. Sci. 2017, 10 (11), 23342341.

137. Tamura, K.; Akutagawa, N.; Satoh, M.; Wada, J.; Masuda, T., Charge/Discharge Properties of Organometallic Batteries Fabricated with Ferrocene-Containing Polymers. Macromol. Rapid Commun. 2008, 29 (24), 1944-1949.

138. Nesvadba, P.; Folger, L. B.; Maire, P.; Novák, P., Synthesis of a Polymeric 2, 5-di-t-butyl-1, 4Dialkoxybenzene and Its Evaluation as a Novel Cathode Material. Synth. Met. 2011, 161 (3-4), 259-262.

139. Man, Z.; Li, P.; Zhou, D.; Zang, R.; Wang, S.; Li, P.; Liu, S.; Li, X.; Wu, Y.; Liang, X., High-

Performance Lithium-Organic Batteries by Achieving 16 Lithium Storage in Poly (imine-anthraquinone). J. Mater. Chem. A. 2019, 7 (5), 2368-2375.

140. Wang, S.; Wang, L.; Zhu, Z.; Hu, Z.; Zhao, Q.; Chen, J., All Organic Sodium-Ion Batteries with $\mathrm{Na}_{4} \mathrm{C}_{8} \mathrm{H}_{2} \mathrm{O}_{6}$. Angew. Chem. Int. Ed. 2014, 53 (23), 5892-5896.

141. Wang, Y.; Ding, Y.; Pan, L.; Shi, Y.; Yue, Z.; Shi, Y.; Yu, G., Understanding the SizeDependent Sodium Storage Properties of $\mathrm{Na}_{2} \mathrm{C}_{6} \mathrm{O}_{6}$-Based Organic Electrodes for Sodium-Ion batteries. Nano Lett. 2016, 16 (5), 3329-3334.

142. Kim, H.; Kwon, J. E.; Lee, B.; Hong, J.; Lee, M.; Park, S. Y.; Kang, K., High Energy Organic Cathode for Sodium Rechargeable Batteries. Chem. Mater. 2015, 27 (21), 7258-7264.

143. Luo, W.; Allen, M.; Raju, V.; Ji, X., An Organic Pigment as a High-Performance Cathode for Sodium-Ion Batteries. Adv. Energy Mater. 2014, 4 (15), 1400554.

144. Banda, H.; Damien, D.; Nagarajan, K.; Raj, A.; Hariharan, M.; Shaijumon, M. M., Twisted Perylene Diimides with Tunable Redox Properties for Organic Sodium-Ion Batteries. Adv. Energy Mater. 2017, 7 (20), 1701316.

145. Deng, W.; Shen, Y.; Qian, J.; Cao, Y.; Yang, H., A Perylene Diimide Crystal with High Capacity and Stable Cyclability for Na-Ion Batteries. ACS Appl. Mater. Interfaces 2015, 7 (38), 2109521099.

146. Hong, J.; Lee, M.; Lee, B.; Seo, D.-H.; Park, C. B.; Kang, K., Biologically Inspired Pteridine Redox Centres for Rechargeable Batteries. Nat. Commun. 2014, 5, 5335.

147. Wang, H.; Yuan, S.; Ma, D.-1.; Huang, X.-1.; Meng, F.-1.; Zhang, X.-b., Tailored Aromatic Carbonyl Derivative Polyimides for High-Power and Long-Cycle Sodium-Organic Batteries. Adv. Energy Mater. 2014, 4 (1301651), 1-7.

148. Banda, H.; Damien, D.; Nagarajan, K.; Hariharan, M.; Shaijumon, M. M., A Polyimide Based All-Organic Sodium Ion Battery. J. Mater. Chem. A. 2015, 3 (19), 10453-10458.

149. Wang, H. g.; Yuan, S.; Ma, D. 1.; Huang, X. 1.; Meng, F. 1.; Zhang, X. b., Tailored Aromatic Carbonyl Derivative Polyimides for High-Power and Long-Cycle Sodium-Organic Batteries. Adv. Energy Mater. 2014, 4 (7), 1301651.

150. Xu, F.; Wang, H.; Lin, J.; Luo, X.; Cao, S.-A.; Yang, H., Poly(anthraquinonyl imide) as a High Capacity Organic Cathode Material for Na-Ion Batteries. J. Mater. Chem. A. 2016, 4 (29), 11491-11497.

151. Xu, F.; Xia, J.; Shi, W., Anthraquinone-Based Polyimide Cathodes for Sodium Secondary Batteries. Electrochem. Commun. 2015, 60, 117-120.

152. Shen, Y.; Yuan, D.; Ai, X.; Yang, H.; Zhou, M., Poly(diphenylaminesulfonic acid sodium) as a Cation-Exchanging Organic Cathode for Sodium Batteries. Electrochem. Commun. 2014, 49, 5-8.

153. Zhou, M.; Li, W.; Gu, T.; Wang, K.; Cheng, S.; Jiang, K., A Sulfonated Polyaniline with High Density and High Rate Na-Storage Performances as a Flexible Organic Cathode for Sodium Ion Batteries. Chem. Commun. 2015, 51 (76), 14354-14356.

154. Luo, C.; Zhu, Y.; Xu, Y.; Liu, Y.; Gao, T.; Wang, J.; Wang, C., Graphene Oxide Wrapped Croconic Acid Disodium Salt for Sodium Ion Battery Electrodes. J. Power Sources 2014, 250, 372-378. 
155. Park, Y.; Shin, D. S.; Woo, S. H.; Choi, N. S.; Shin, K. H.; Oh, S. M.; Lee, K. T.; Hong, S. Y., Sodium Terephthalate as an Organic Anode Material for Sodium Ion Batteries. Adv. Mater. 2012, 24 (26), 3562-3567.

156. Wang, C.; Xu, Y.; Fang, Y.; Zhou, M.; Liang, L.; Singh, S.; Zhao, H.; Schober, A.; Lei, Y., Extended $\pi$-Conjugated System for Fast-Charge and-Discharge Sodium-Ion Batteries. J. Am. Chem. Soc. 2015, 137 (8), 3124-3130.

157. Choi, A.; Kim, Y. K.; Kim, T. K.; Kwon, M.-S.; Lee, K. T.; Moon, H. R., 4, 4'Biphenyldicarboxylate Sodium Coordination Compounds as Anodes for $\mathrm{Na}-$ Ion Batteries. J. Mater. Chem. A 2014, 2 (36), 14986-14993.

158. Mihali, V. A.; Renault, S.; Nyholm, L.; Brandell, D., Benzenediacrylates as Organic Battery Electrode Materials: Na Versus Li. RSC Adv. 2014, 4 (72), 38004-38011.

159. Zhu, Z.; Li, H.; Liang, J.; Tao, Z.; Chen, J., The Disodium Salt of 2, 5-Dihydroxy-1,4Benzoquinone as Anode Material for Rechargeable Sodium Ion Batteries. Chem. Commun. 2015, 51 (8), 1446-1448.

160. Wang, H.; Hu, P.; Yang, J.; Gong, G.; Guo, L.; Chen, X., Renewable-Juglone-Based HighPerformance Sodium-Ion Batteries. Adv. Mater. 2015, 27 (14), 2348-2354.

161. Renault, S.; Mihali, V. A.; Edström, K.; Brandell, D., Stability of Organic Na-Ion Battery Electrode Materials: the Case of Disodium Pyromellitic Diimidate. Electrochem. Commun. 2014, 45, 5255.

162. Wang, H.-g.; Yuan, S.; Si, Z.; Zhang, X.-b., Multi-Ring Aromatic Carbonyl Compounds Enabling High Capacity and Stable Performance of Sodium-Organic Batteries. Energy Environ. Sci. 2015, $8(11), 3160-3165$.

163. López-Herraiz, M.; Castillo-Martínez, E.; Carretero-González, J.; Carrasco, J.; Rojo, T.; Armand, M., Oligomeric-Schiff Bases as Negative Electrodes for Sodium Ion Batteries: Unveiling the Nature of Their Active Redox Centers. Energy Environ. Sci. 2015, 8 (11), 3233-3241.

164. Chen, L.; Li, W.; Wang, Y.; Wang, C.; Xia, Y., Polyimide as Anode Electrode Material for Rechargeable Sodium Batteries. RSC Adv. 2014, 4 (48), 25369-25373.

165. Deng, W.; Liang, X.; Wu, X.; Qian, J.; Cao, Y.; Ai, X.; Feng, J.; Yang, H., A Low Cost, AllOrganic Na-Ion Battery Based on Polymeric Cathode and Anode. Sci. Rep. 2013, 3, 2671.

166. Zhou, D.; Chen, Y.; Li, B.; Fan, H.; Cheng, F.; Shanmukaraj, D.; Rojo, T.; Armand, M.; Wang, G., A Stable Quasi-Solid-State Sodium-Sulfur Battery. Angew. Chem. Int. Ed. 2018, 57 (32), 1016810172.

167. Chen, Y.; Luo, W.; Carter, M.; Zhou, L.; Dai, J.; Fu, K.; Lacey, S.; Li, T.; Wan, J.; Han, X., Organic Electrode for Non-Aqueous Potassium-Ion Batteries. Nano Energy 2015, 18, 205-211.

168. Fan, L.; Ma, R.; Wang, J.; Yang, H.; Lu, B., An Ultrafast and Highly Stable Potassium-Organic Battery. Adv. Mater. 2018, 30 (51), 1805486.

169. Liang, Y.; Luo, C.; Wang, F.; Hou, S.; Liou, S.-C.; Qing, T.; Li, Q.; Zheng, J.; Cui, C.; Wang, C., An Organic Anode for High Temperature Potassium-Ion Batteries. Adv. Energy Mater.s 2019, 9 (2), 1802986.

170. Deng, Q.; Pei, J.; Fan, C.; Ma, J.; Cao, B.; Li, C.; Jin, Y.; Wang, L.; Li, J., Potassium Salts of Para-Aromatic Dicarboxylates as the Highly Efficient Organic Anodes for Low-Cost K-Ion Batteries. Nano Energy 2017, 33, 350-355.

171. Zhao, J.; Yang, J.; Sun, P.; Xu, Y., Sodium Sulfonate Groups Substituted Anthraquinone as An Organic Cathode for Potassium Batteries. Electrochem. Commun. 2018, 86, 34-37.

172. Xue, Q.; Li, D.; Huang, Y.; Zhang, X.; Ye, Y.; Fan, E.; Li, L.; Wu, F.; Chen, R., Vitamin K as a High-performance Organic Anode Material for Rechargeable Potassium Ion Batteries. J. Mater. Chem. A 2018, 6 (26), 12559-12564.

173. Jian, Z.; Liang, Y.; Rodríguez-Pérez, I. A.; Yao, Y.; Ji, X., Poly(anthraquinonyl sulfide) Cathode for Potassium-Ion Batteries. Electrochem. Commun. 2016, 71, 5-8.

174. Rodríguez-Pérez, I. A.; Yuan, Y.; Bommier, C.; Wang, X.; Ma, L.; Leonard, D. P.; Lerner, M. M.; Carter, R. G.; Wu, T.; Greaney, P. A.; Lu, J.; Ji, X., Mg-Ion Battery Electrode: An Organic Solid's Herringbone Structure Squeezed upon Mg-Ion Insertion. J. Am. Chem. Soc. 2017, 139 (37), 1303113037.

175. Pan, B.; Zhou, D.; Huang, J.; Zhang, L.; Burrell, A. K.; Vaughey, J. T.; Zhang, Z.; Liao, C., 2, 5Dimethoxy-1, 4-Benzoquinone (DMBQ) as Organic Cathode for Rechargeable Magnesium-Ion Batteries. J. Electrochem. Soc. 2016, 163 (3), A580-A583.

176. Pan, B.; Huang, J.; Feng, Z.; Zeng, L.; He, M.; Zhang, L.; Vaughey, J. T.; Bedzyk, M. J.; Fenter, P.; Zhang, Z.; Burrell, A. K.; Liao, C., Polyanthraquinone-Based Organic Cathode for High-Performance Rechargeable Magnesium-Ion Batteries. Adv. Energy Mater. 2016, 6 (14), 1600140. 
177. Bitenc, J.; Pirnat, K.; Bančič, T.; Gaberšček, M.; Genorio, B.; Randon-Vitanova, A.; Dominko, R., Anthraquinone-Based Polymer as Cathode in Rechargeable Magnesium Batteries. ChemSusChem 2015, 8 (24), 4128-4132.

178. Dong, H.; Liang, Y.; Tutusaus, O.; Mohtadi, R.; Zhang, Y.; Hao, F.; Yao, Y., Directing MgStorage Chemistry in Organic Polymers toward High-Energy Mg Batteries. Joule 2019, 3, 782-793.

179. Wang, F.; Fan, X.; Gao, T.; Sun, W.; Ma, Z.; Yang, C.; Han, F.; Xu, K.; Wang, C., High-Voltage Aqueous Magnesium Ion Batteries. ACS Cent. Sci. 2017, 3 (10), 1121-1128.

180. Lu, D.; Liu, H.; Huang, T.; Xu, Z.; Ma, L.; Yang, P.; Qiang, P.; Zhang, F.; Wu, D., Magnesium Ion Based Organic Secondary Batteries. J. Mater. Chem. A 2018, 6 (36), 17297-17302.

181. Bančič, T.; Bitenc, J.; Pirnat, K.; Kopač Lautar, A.; Grdadolnik, J.; Randon Vitanova, A.; Dominko, R., Electrochemical Performance and Redox Mechanism of Naphthalene-Hydrazine Diimide Polymer as a Cathode in Magnesium Battery. J. Power Sources 2018, 395, 25-30.

182. Kundu, D.; Oberholzer, P.; Glaros, C.; Bouzid, A.; Tervoort, E.; Pasquarello, A.; Niederberger, M., Organic Cathode for Aqueous Zn-Ion Batteries: Taming a Unique Phase Evolution toward Stable Electrochemical Cycling. Chem. Mater. 2018, 30 (11), 3874-3881.

183. Guo, Z.; Ma, Y.; Dong, X.; Huang, J.; Wang, Y.; Xia, Y., An Environmentally Friendly and Flexible Aqueous Zinc Battery Using an Organic Cathode. Angew. Chem. 2018, 130 (36), 11911-11915.

184. Zhao, Q.; Huang, W.; Luo, Z.; Liu, L.; Lu, Y.; Li, Y.; Li, L.; Hu, J.; Ma, H.; Chen, J., HighCapacity Aqueous Zinc Batteries Using Sustainable Quinone Electrodes. Sci. Adv. 2018, 4 (3), eaao1761/1-eaao1761/10.

185. Dawut, G.; Lu, Y.; Miao, L.; Chen, J., High-Performance Rechargeable Aqueous Zn-Ion Batteries with a Poly(benzoquinonyl sulfide) Cathode. Inorganic Chemistry Frontiers 2018, 5 (6), 13911396.

186. Häupler, B.; Rössel, C.; Schwenke, A. M.; Winsberg, J.; Schmidt, D.; Wild, A.; Schubert, U. S., Aqueous Zinc-Organic Polymer Battery with a High Rate Performance and Long Lifetime. NPG Asia Mater. 2016, 8, e283.

187. Wan, F.; Zhang, L.; Wang, X.; Bi, S.; Niu, Z.; Chen, J., An Aqueous Rechargeable Zinc-Organic Battery with Hybrid Mechanism. Adv. Funct. Mater. 2018, 28 (45), 1804975.

188. Gheytani, S.; Liang, Y.; Wu, F.; Jing, Y.; Dong, H.; Rao, K. K.; Chi, X.; Fang, F.; Yao, Y., An Aqueous Ca-Ion Battery. Adv. Sci. 2017, 4 (12), 1700465.

189. Kim, D. J.; Yoo, D.-J.; Otley, M. T.; Prokofjevs, A.; Pezzato, C.; Owczarek, M.; Lee, S. J.; Choi, J. W.; Stoddart, J. F., Rechargeable Aluminium Organic Batteries. Nat. Energy 2019, 4 (1), 51-59.

190. Hudak, N. S., Chloroaluminate-Doped Conducting Polymers as Positive Electrodes in Rechargeable Aluminum Batteries. J. Phys. Chem. C 2014, 118 (10), 5203-5215.

191. Walter, M.; Kravchyk, K. V.; Böfer, C.; Widmer, R.; Kovalenko, M. V., Polypyrenes as HighPerformance Cathode Materials for Aluminum Batteries. Adv. Mater. 2018, 30 (15), 1705644.

192. Li, C.; Zhang, H.; Otaegui, L.; Singh, G.; Armand, M.; Rodriguez-Martinez, L. M., Estimation of Energy Density of Li-S Batteries with Liquid and Solid Electrolytes. J. Power Sources 2016, 326, 1-5. 193. Judez, X.; Zhang, H.; Li, C.; Eshetu, G. G.; González-Marcos, J. A.; Armand, M.; RodriguezMartinez, L. M., Solid Electrolytes for Safe and High Energy Density Lithium-Sulfur Batteries: Promises and Challenges. J. Electrochem. Soc. 2018, 165 (1), A6008-A6016.

194. Nitta, N.; Wu, F.; Lee, J. T.; Yushin, G., Li-Ion Battery Materials: Present and Future. Mater. Today 2015, 18 (5), 252-264.

195. Nakano, H.; Dokko, K.; Koizumi, S.; Tannai, H.; Kanamura, K., Hydrothermal Synthesis of Carbon-Coated $\mathrm{LiFePO}_{4}$ and Its Application to Lithium Polymer Battery. J. Electrochem. Soc. 2008, 155 (12), A909-A914.

196. Li, X.; Kang, F.; Bai, X.; Shen, W., A Novel Network Composite Cathode of LiFePO4/Multiwalled Carbon Nanotubes with High Rate Capability for Lithium Ion Batteries. Electrochem. Commun. 2007, 9 (4), 663-666.

197. Song, J.; Sun, B.; Liu, H.; Ma, Z.; Chen, Z.; Shao, G.; Wang, G., Enhancement of the Rate Capability of $\mathrm{LiFePO}_{4}$ by a New Highly Graphitic Carbon-Coating Method. ACS Appl. Mater. Interfaces 2016, 8 (24), 15225-15231.

198. Zhou, X.; Wang, F.; Zhu, Y.; Liu, Z., Graphene Modified $\mathrm{LiFePO}_{4}$ Cathode Materials for High Power Lithium Ion Batteries. J. Mater. Chem. 2011, 21 (10), 3353-3358.

199. Song, Z.; Xu, T.; Gordin, M. L.; Jiang, Y.-B.; Bae, I.-T.; Xiao, Q.; Zhan, H.; Liu, J.; Wang, D., Polymer-Graphene Nanocomposites as Ultrafast-Charge and-Discharge Cathodes for Rechargeable Lithium Batteries. Nano Lett. 2012, 12 (5), 2205-2211.

200. Cheng, Y.-Y.; Li, C.-C.; Lee, J.-T., Electrochemical Behavior Of Organic Radical Polymer Cathodes in Organic Radical Batteries with $N$-Butyl- $N$-methylpyrrolidinium Bis(trifluoromethylsulfonyl)imide Ionic Liquid Electrolytes. Electrochim. Acta 2012, 66, 332-339. 
201. Muldoon, J.; Bucur, C. B.; Gregory, T., Quest for Nonaqueous Multivalent Secondary Batteries: Magnesium and Beyond. Chem. Rev. 2014, 114 (23), 11683-11720.

202. Yang, H.; Li, H.; Li, J.; Sun, Z.; He, K.; Cheng, H.-M.; Li, F., The Rechargeable Aluminum Battery: Opportunities and Challenges. Angew. Chem. Int. Ed. 2019. DOI: 10.1002/ange.201814031 